\title{
HYDROLOGY OF THE L.C. HOLDING COAL-LEASE TRACT AND ADJACENT AREAS, SOUTHWESTERN UTAH, AND POTENTIAL EFFECTS OF COAL MINING
}

By G.E. Cordy, R.L. Seiler, and B.J. Stolp

U.S. GEOLOGICAL SURVEY

Water-Resources Investigations Report 91-4111

Prepared in cooperation with the

U.S. BUREAU OF LAND MANAGEMENT

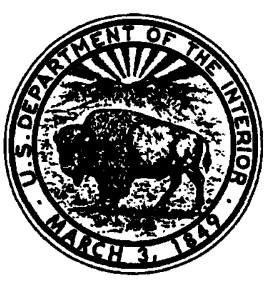

Salt Lake City, Utah 


\section{U.S. DEPARTMENT OF THE INTERIOR \\ BRUCE BABBITT, Secretary \\ U.S. GEOLOGICAL SURVEY}

Robert M. Hirsch, Acting Director

For additional information write to:

District Chief

U.S. Geological Survey

1016 Administration Building

1745 West 1700 South

Salt Lake City, Utah 84104
Copies of the report can be purchased from:

U.S. Geological Survey Earth Science Information Center Open-File Reports Section Box 25286, MS 517 Denver Federal Center Denver, Colorado 80225 
Abstract $\ldots \ldots \ldots \ldots \ldots \ldots \ldots \ldots \ldots \ldots \ldots \ldots \ldots \ldots \ldots \ldots \ldots \ldots \ldots \ldots \ldots$

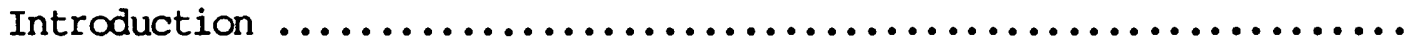

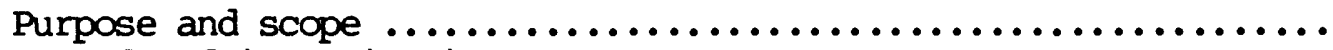

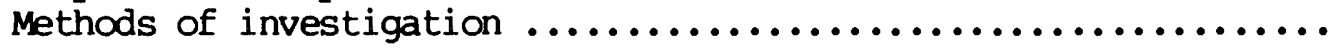

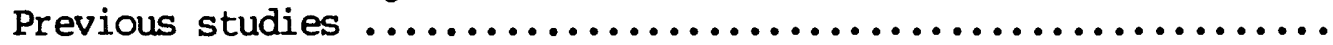

Data-site numbering system $\ldots \ldots \ldots \ldots \ldots \ldots \ldots \ldots \ldots \ldots \ldots \ldots \ldots \ldots$

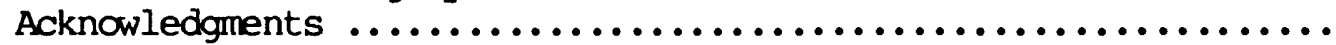

Physical setting of lease tract and vicinity $\ldots \ldots \ldots \ldots \ldots \ldots \ldots \ldots$

Location and topography $\ldots \ldots \ldots \ldots \ldots \ldots \ldots \ldots \ldots \ldots \ldots \ldots$

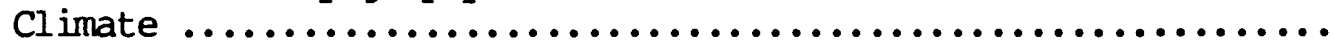

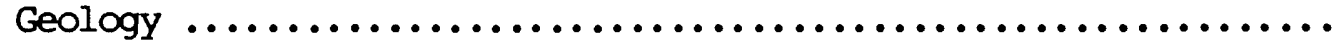

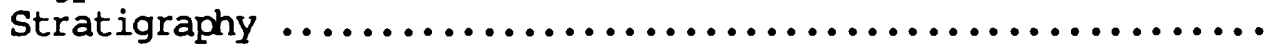

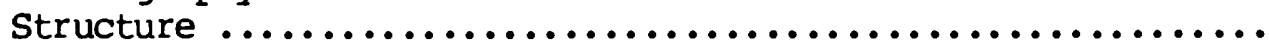

Landslide hazards $\ldots \ldots \ldots \ldots \ldots \ldots \ldots \ldots \ldots \ldots \ldots \ldots \ldots \ldots \ldots \ldots$

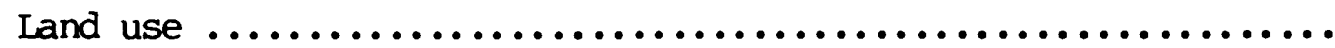

Surface-water hydrology $\ldots \ldots \ldots \ldots \ldots \ldots \ldots \ldots \ldots \ldots \ldots \ldots \ldots \ldots \ldots \ldots \ldots$

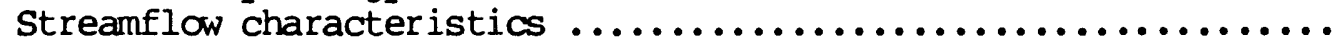

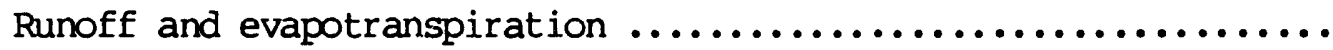

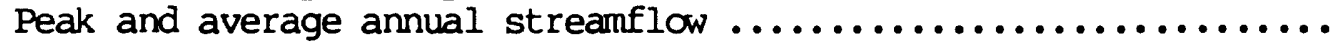

Water quality $\ldots \ldots \ldots \ldots \ldots \ldots \ldots \ldots \ldots \ldots \ldots \ldots \ldots \ldots \ldots \ldots \ldots$

Chemical quality $\ldots \ldots \ldots \ldots \ldots \ldots \ldots \ldots \ldots \ldots \ldots \ldots \ldots \ldots \ldots \ldots$

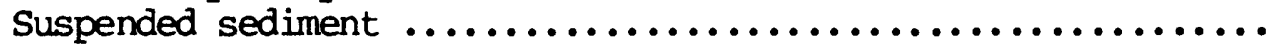

Erosion and sediment yield $\ldots \ldots \ldots \ldots \ldots \ldots \ldots \ldots \ldots \ldots \ldots \ldots$

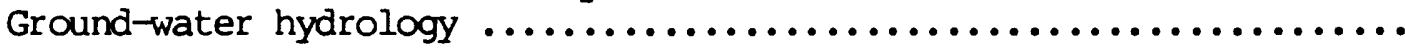

Occurrence of ground water $\ldots \ldots \ldots \ldots \ldots \ldots \ldots \ldots \ldots \ldots \ldots \ldots \ldots$

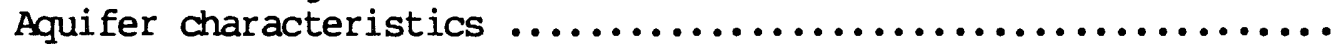

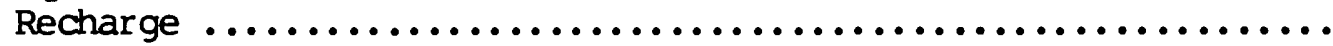

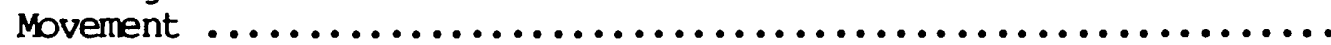

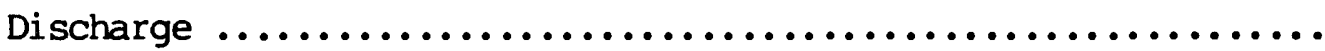

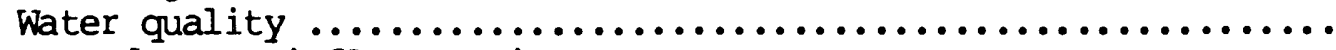

Ground-water inflow to $\mathrm{Zion}$ Narrows $\ldots \ldots \ldots \ldots \ldots \ldots \ldots \ldots \ldots \ldots$

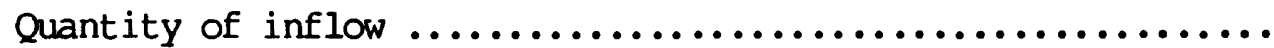

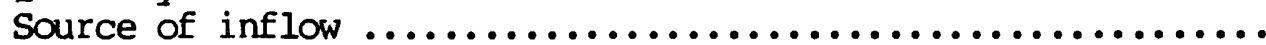

Surface-water/ground-water interaction $\ldots \ldots \ldots \ldots \ldots \ldots \ldots \ldots \ldots \ldots$

Potential effects of mining $\ldots \ldots \ldots \ldots \ldots \ldots \ldots \ldots \ldots \ldots \ldots \ldots \ldots \ldots$

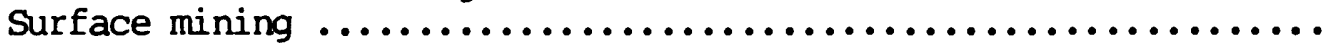

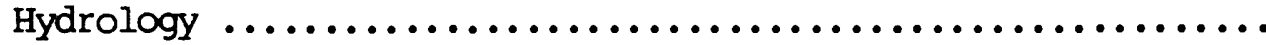

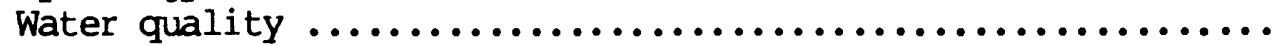

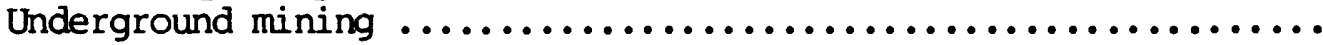

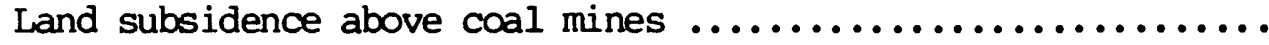

Potential effects of subsidence in the lease tract ..........

Future studies needed $\ldots \ldots \ldots \ldots \ldots \ldots \ldots \ldots \ldots \ldots \ldots \ldots \ldots \ldots \ldots \ldots \ldots$

Sumary and conclusions $\ldots \ldots \ldots \ldots \ldots \ldots \ldots \ldots \ldots \ldots \ldots \ldots \ldots \ldots \ldots \ldots$

References cited ......................................

Supplemental information $\ldots \ldots \ldots \ldots \ldots \ldots \ldots \ldots \ldots \ldots \ldots \ldots \ldots \ldots \ldots$ 


\section{ILLUSTRATIONS}

[Plates are in pocket]

Plate 1. Map showing geology, spring locations, and waterquality diagrams for the L.C. Holding coal-lease tract area, Utah.

2. Map showing areas of ground instability and location of monitoring wells and streamflow-sampling sites for the L.C. Holding coal-lease tract area, Utah.

Figure 1. Map showing location of the L.C. Holding

Page

coal-lease tract ..............................

2. Diagram showing data-site numbering system used

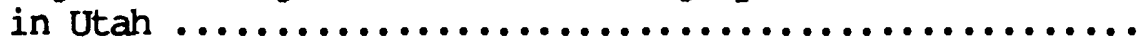

3. Photograph showing vegetation and slopes typical of the L.C. Holding coal-lease tract ...............

4. Graphs showing mean monthly precipitation and temperature at Alton and Zion, 1951-86 .............

5. Satellite imagery showing structural features near the L.C. Holding coal-lease tract ..............

6. Photographs showing joint systems in the Navajo Sandstone

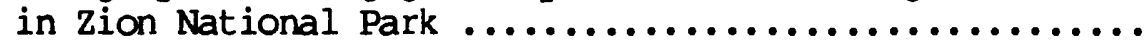

7. Photograph showing slump in coal-bearing member of the Tropic Shale near site swl $6 \ldots \ldots \ldots \ldots \ldots \ldots \ldots \ldots$

8. Photograph showing displacement of pavement by coal Hill landslide ...............................

9. Map showing location of and selected water-quality diagrams for springs, streamflow sites, and a stable isotope sample site in zion Canyon .................

10. Graphs showing mean monthly precipitation at Alton and mean monthly streamflow at two gaging stations

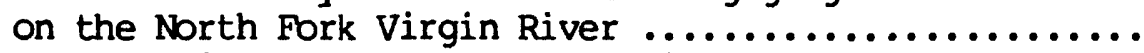

11. Graph showing mean monthly precipitation at Alton and mean monthly runoff at North Fork Virgin River below Bulloch Canyon, SW2, 1975-83 ................

12. Graph showing relation of streamflow to suspendedsediment concentration at site SW2, North Fork Virgin River below Bulloch Canyon, October 1974 through

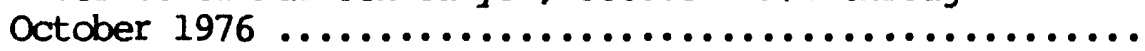

13. Photographs showing sediment load in the North Fork Virgin River before (A) and during (B) thunderstorm runoff

14. Map showing location of and water-quality diagrams for wells and springs outside the L.C. Holding

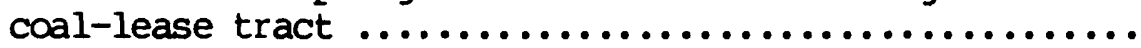

15. Map showing location and elevation of selected springs

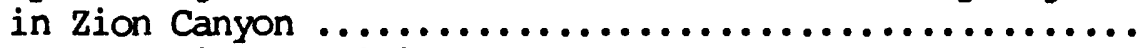

16. Graph showing specific conductance for samples from springs discharging from the Navajo aquifer,

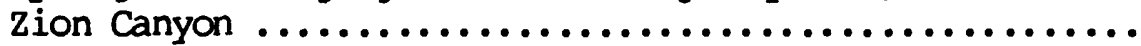

17. Graph showing cumulative departure from normal precipitation at Zion and Alton, 1967-87 ............ 
Table 1. Streamflow, temperature, specific conductance, and suspended-sediment concentration for selected streamflow sites .............................

2. Regression equations used to compute peak and average annual streamflows from ungaged tributaries in the L.C.

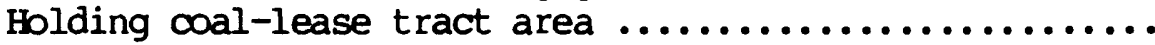

3. Streamflow and chemical quality of water from selected

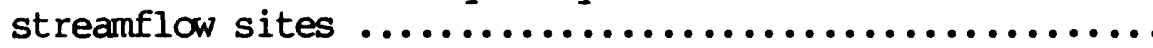

4. Streamflow, suspended-sediment concentration, and particle-size distribution for selected streamflow

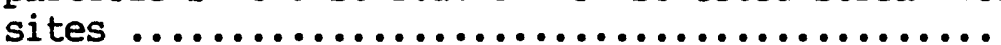

5. Rating factors for estimating source-area sediment yield for the L.C. Holding coal-lease tract ............

6. Estimates of suspended-sediment load for gaging stations along the North Fork Virgin River below Bulloch Canyon

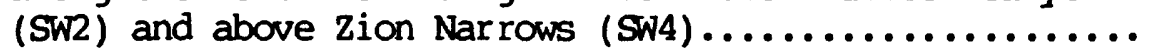

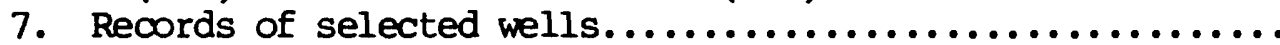

8. Discharge and chemical quality of water from selected

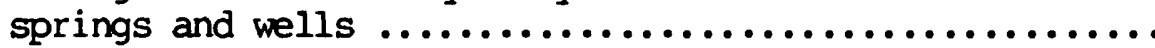

9. Compilation of discharge data for springs in and adjacent to the L.C. Holding coal-lease tract ......... 58

10. Chemical analyses for dissolved metals and stable isotopes in water from selected springs and wells ....... 
CONVERSION FACTORS, ABBREVIATED WATER-QUALITY UNITS, AND VERTICAL DATUM

\section{Multiply}

foot ( $\mathrm{ft})$
inch (in.)
mile (mi)
yard ( $\mathrm{yd})$
acre
square foot $\left(\mathrm{ft}^{2}\right)$
square mile $\left(\mathrm{mi}^{2}\right)$

acre-foot (acre-ft)

cubic foot per second ( $\mathrm{ft}^{3} / \mathrm{s}$ )

gallon per minute (gal/min)

acre-foot per square mile (acre-ft/mi ${ }^{2}$ )
By

Length

0.3048

2.54

1.609

0.9144

\section{Area}

0.4047

0.09290

2.59

Volume

0.001233

Flow

0.02882

0.06309

$\underline{\text { Yield }}$

0.0004761

Density

tons per acre-foot (tons/acre-ft) $\quad 0.00136$
To obtain

meter

centimeter

kilameter

meter

square hectameter

square meter

square kilometer

cubic hectometer

cubic meter per second liter per second

cubic hectometer per square kilometer

\section{Specific Capacity}

gallon per minute per foot [(gal/min)/ft]

0.20696

liter per second per meter

Hydraulic Conductivity

foot per day (ft/day)

0.0003528

centimeter per second

Transmissivity

foot squared per day ${ }^{1}\left(\mathrm{ft}^{2} /\right.$ day) $\quad 0.09290 \quad$ meter squared per day

1 An alternative way of expressing transmissivity is cubic foot per day per square foot per foot of aquifer thickness [(ft $\left./ \mathrm{d} / \mathrm{ft}^{2}\right) / \mathrm{ft}$ ]. 
Gradient

foot per mile $(\mathrm{ft} / \mathrm{mi}) \quad 0.1894 \quad$ meter per kilameter

Concentrations of chemical constituents are given in milligrams per liter $(\mathrm{mg} / \mathrm{L})$ or micrograms per liter $(\mu \mathrm{g} / \mathrm{L})$. Milligrams per liter is a unit expressing the concentration of chemical constituent as the weight (milligrams) of solute per unit volume (liter of water). One thousand micrograms per liter is equivalent to 1 milligram per liter. The concentration of chemical constituents, in parts per million, is about the same as the concentration in milligrams per liter for concentrations less than $7,000 \mathrm{mg} / \mathrm{L}$. $(\mu \mathrm{S} / \mathrm{cm})$.

Specific conductance is given in microsiemens per centimeter at $25^{\circ} \mathrm{C}$

Water and air temperature are given in degrees celsius $\left({ }^{\circ} \mathrm{C}\right)$, which can be converted to degrees Fahrenheit $\left({ }^{\circ} \mathrm{F}\right)$ by the following equation:

$$
{ }^{\circ} \mathrm{F}=1.8\left({ }^{\circ} \mathrm{C}\right)+32 .
$$

Sea level: In this report "sea level" refers to the National Geodetic Vertical Datum of 1929-a geodetic datum derived from a general adjustment of the first-order level nets of the United States and Canada, formerly called Sea Level Datum of 1929. 


\title{
HYDROLOGY OF THE L.C. HOLDING COAL-LEASE TRACT
}

AND ADJACENT AREAS, SOUTHWESTERN UTAH, AND POTENITIAL EFFECTS OF COAL MINING

by G.E. Cordy, R.L. Seiler, and B.J. Stolp

\begin{abstract}
In preparing an Environmental Impact Statement, the U.S. Bureau of Land Management recognized a need for baseline hydrologic data and an understanding of the hydrologic system in the L.C. Holding coal-lease tract in southwestern Utah in order to determine the potential effects of mining on the water resources of the area. The potential effects of mining on the hydrology of the area were of particular concern because Zion National Park is less than 3 miles from the westernmost part of the lease tract. Much of the water that passes through the Park either originates in the lease tract or traverses it.
\end{abstract}

Base flow in the major perennial stream in the area, the North Fork Virgin River, and several of its tributaries is sustained by spring discharge and diffuse seepage. Runoff from melting snowpacks and summer thundershowers contributes most of the flow to perennial streams.

Water in the North Fork Virgin River has a dissolved-solids concentration ranging from 190 to 280 milligrams per liter, and the chemical composition is about the same from upstream to downstream sample sites despite the inflow of water with larger dissolved-solids concentrations from some tributaries. Suspended-sediment concentrations fluctuate with discharge. The largest concentrations occur during runoff from intense thunderstorms in surmer and fall.

Regional ground-water movement is generally southward. Most of the geologic formations in the study area contain aquifers. The water table in the regional aquifer is about 870 feet below land surface in the Navajo Sandstone. Ground water issuing from the Navajo Sandstone on the east side of Zion Canyon has specific conductance values several times larger than ground water from the west side, indicating recharge to the Navajo from the overlying strata, which contain water of larger specific conductance.

Potential effects of mining in the area include: (1) Increased dissolved-solids concentrations and decreased pH values in both surface and ground water; (2) dewatering of aquifers, causing decrease or cessation of flow to some springs; and (3) land subsidence and associated subsidence fractures.

\section{INIRODUCTION}

The L.C. Holding coal-lease tract is in southwestern Utah in the Kolob coal field (fig. 1). The lease tract is comprised of three lease areas that cover about 19,000 acres. The U.S. Bureau of Land Management (1986) estimates that Federal coal reserves in the lease tract total 209 billion 


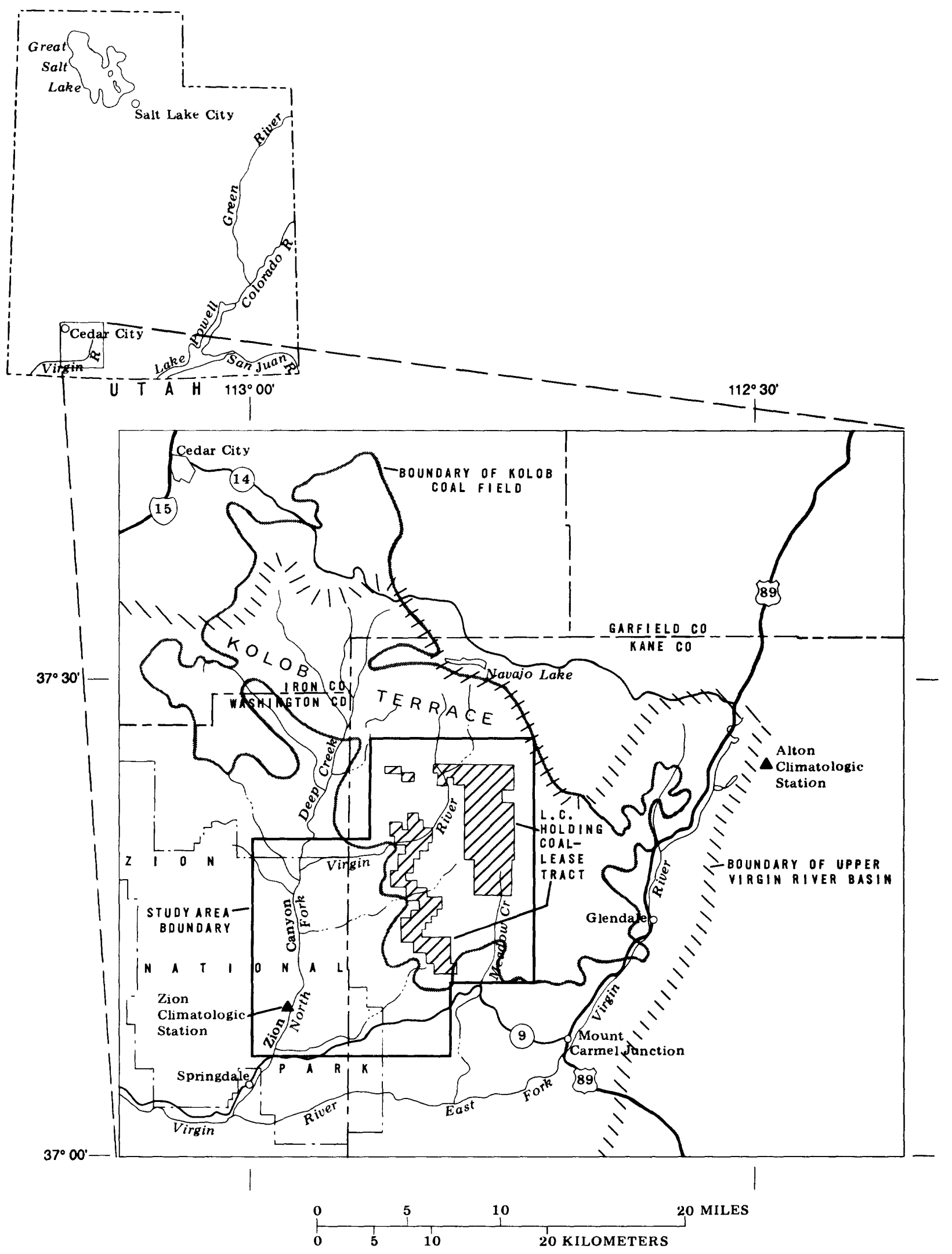

Figure 1.--Location of the L.C. Holding coal-lease tract. 
tons. Except for a few small prospecting claims, coal has not been mined commercially in this part of Utah.

The U.S. Bureau of Land Management (USBLM), in preparing an Environmental Impact Statement for potential mining in the L.C. Holding coal-lease tract, reoognized a need for baseline hydrologic data and an understanding of the hydrologic system in the lease tract in order to determine the potential effects of mining on the water resources of the area. To provide such information, the U.S. Geological Survey (USGS), in cooperation with USBLM, conducted a hydrologic study of the L.C. Holding coal-lease tract and adjacent areas from October 1985 through January 1987. The primary purpose of the study was to provide USBLM with baseline hydrologic information so they can make informed decisions about the development of the coal-lease tract.

\section{Purpose and Scope}

This report presents the results of a study of the hydrology of the L.C. Holding coal-lease tract and adjacent areas and the potential effects of coal mining on the hydrology of the area. In order to determine the potential effects of mining on the hydrologic system of the area, the baseline (premining) hydrologic characteristics were established. Information on the quantity of base flow contributed by the coal-lease tract and adjacent areas to the North Fork Virgin River and to springs in Zion National Park was derived from the baseline data.

\section{Methods of Investigation}

Data were collected from June 1986 to October 1987. Data needed to characterize the ground-water system were obtained by: (1) Compiling data fram previous studies; (2) measuring streamflow and determining ground-water seepage to streams; (3) making an inventory of springs, spring discharge, wells, and water levels; (4) analyzing the chemical quality of water from springs, wells, and streams; (5) determining suspended-sediment concentrations in streams; and (6) drilling observation wells. In addition, erosion and sediment-yield data as well as landslide locations were compiled and used to evaluate aspects of the potential effects of coal mining in the area.

Regression equations developed for Utah streams (Christensen and others, 1986; Thomas and Lindskov, 1983) were used to estimate peak and average streamflow from ungaged drainages. Sediment yields from the study area were estimated using the Pacific Southwest Inter-Agency Committee (PSIAC)(1968) method.

Because of the need to understand the effects of mining on the hydrologic system beyond the coal-lease boundaries, particularly in Zion National Park, data were ollected from the area surrounding the lease tract, including Zion Canyon in the Park. The study area is shown in figure 1. References to the L.C. Holding coal-lease tract in this report are specifically limited to the area within the lease-tract boundaries shown on plates 1 and 2 . 


\section{Previous Studies}

General watershed conditions in the upper Virgin River basin, which includes the lease tract, were discussed by Turner (1949). A hydrologic reconnaissance of the coal fields of south-central Utah by Plantz (1985) described the general ground-water and surface-water conditions in the Kolob coal field. A general overview of the hydrology of the lease tract and the surrounding area was included in a compilation of the regional hydrology of Area 57, northern Great Plains and Rocky Mountain coal provinces (Price, 1987).

Gates (1965) described the local ground-water conditions determined from a well at the east entrance to Zion National Park, about $4 \mathrm{mi}$ southwest of the lease tract. Ground-water conditions in the upper Virgin River basin were detailed by Cordova (1981).

The chemical quality of surface water in the kolob coal field was shown on a map by Price (1980). Selected data on runoff and surface-water discharge for the same area were presented by Price (1983). Results of a study of surface-water quality in the upper Virgin River basin were described by Sandberg and Sultz (1985).

The geology and geography of the lease tract and surrounding area were described in a paper on the Zion National Park region by Gregory (1950). The study area is included on a geologic map by Cashion (1967) that was used as a reference for this study. Geophysical logs for 14 coal test holes in the lease tract and adjacent areas are described in a report by Bowers and Strickland (1978). General information on landslide hazards in the area was presented by Colton (1976). Umpublished maps showing existing and potential areas of landslides and ground instability in the study area were provided by Peter M. Kilbourne (geologist, U.S. Bureau of Land Management, written commun., 1987).

\section{Data-Site Numbering System}

The system of numbering wells, springs, and other sites in Utah is based on the cadastral land-survey system of the U.S. Government. The site number, in addition to designating the well or spring, describes its position in the land net. The State is divided into four quadrants by the Salt Lake Base Line and Meridian, and these quadrants are designated by the uppercase letters $A$, $B, C$, and D, indicating the northeast, northwest, southwest, and southeast quadrants, respectively. Numbers designating the township and range (in that order) follow the quadrant letter, and all three are enclosed in parentheses. The number after the parentheses indicates the section and is followed by three letters indicating the quarter section, the quarter-quarter section, and the quarter-quarter-quarter section--generally 10 acres $^{1}$. The lowercase

${ }^{1}$ Although the basic land unit, the section, is theoretically I square $\mathrm{mi}$, many sections are irregular. Such sections are subdivided into 10-acre tracts, generally beginning at the southeast corner, and the surplus or shortage is taken up in the tracts along the north and west sides of the section. Sections have not been designated in much of $\mathrm{Zion}$ National Park; therefore, spring and well numbers used in this report may be erroneous if the area is surveyed in the future. 
letters $a, b, c$, and $d$ indicate, respectively, the northeast, northwest, southwest, and southeast quarters of each subdivision. The number after the letters is the serial number of the spring or well within the 10-acre tract, the letter "S" preceding the serial number denotes a spring. Thus, (C-399) 16 bac-Sl designates the first spring visited in the $S N_{\frac{1}{4}} N_{E}^{\frac{1}{4}} N W_{\frac{1}{4}}$, sec. 16, T. $39 \mathrm{~S} ., \mathrm{R} .9 \mathrm{~W}$. If a well or spring cannot be located within a 10-acre tract, one or two location letters are used and the serial number is omitted. Other sites where hydrologic data were collected are numbered in the same manner, but three letters are used after the section number and no serial number is used. The numbering system is illustrated in figure 2.

\section{Acknowledgments}

Appreciation is extended to Roy Lundgren, Mrs. Spendlove, and the Washington County Water Conservancy District for allowing access to their property for drilling. Special thanks to the local residents, particularly Harold Drews, for providing information on the area and allowing access to wells and springs. Peter M. Kilbourne of the U.S. Bureau of Land Management, Kanab, provided maps and data on landslides in the study area.

\section{PHYSICAL SETTING OF LEASE TRACT AND VICINITY}

\section{Location and Topography}

The L.C. Holding coal-lease tract is in northwestern Kane County (fig. 1). At its southernmost extent, the coal-lease tract is about $2 \mathrm{mi}$ north of State Highway 9 and $2.5 \mathrm{mi}$ east of the Zion National Park boundary at the westernmost extent (fig. 1).

Major streams that drain the area include North Fork Virgin River, Orderville Gulch, Clear Creek, and Meadow Creek (pl. 1). These streams and their tributaries have dissected the area into deep, steep-sided canyons. Canyon walls typically exhibit sheer cliffs of sandstone and vegetated slopes of less resistant shale (fig. 3). Land-surface elevations in the lease tract range from about 5,830 ft where the North Fork Virgin River crosses the western boundary of the tract to $8,200 \mathrm{ft}$ southwest of Pine Knoll (pl. 1) in the northeast corner of the tract.

\section{Climate}

The L.C. Holding coal-lease tract is semiarid with average annual precipitation for the area ranging from 20 to 25 in. (U.S. Weather Bureau, 1963). The area receives most of its moisture in the late summer (July, August, and September) and winter months (November, December, January, February, and March) as indicated by graphs of mean monthly precipitation (fig. 4) measured at gages (fig. 1) in the Alton and Zion Canyon areas (National Oceanic and Atmospheric Administration, 1981-86). Winter precipitation generally comes from frontal storms as snowfall. Summer precipitation usually results from convection thundershowers. 
R. 9 W.

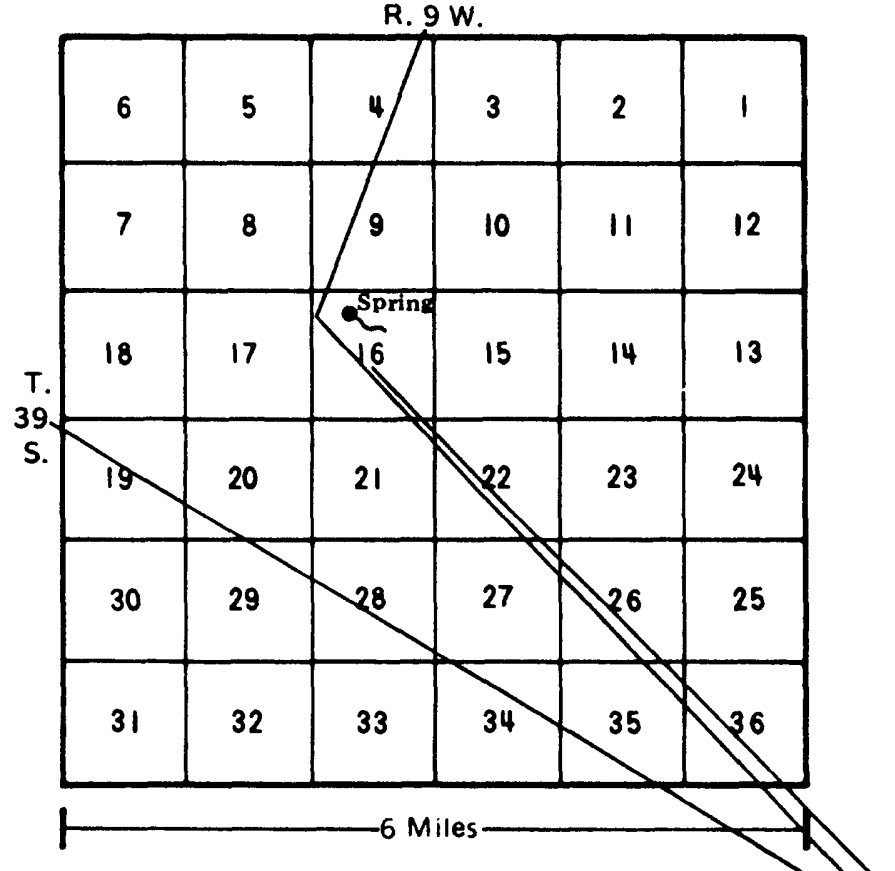

Sec. 16

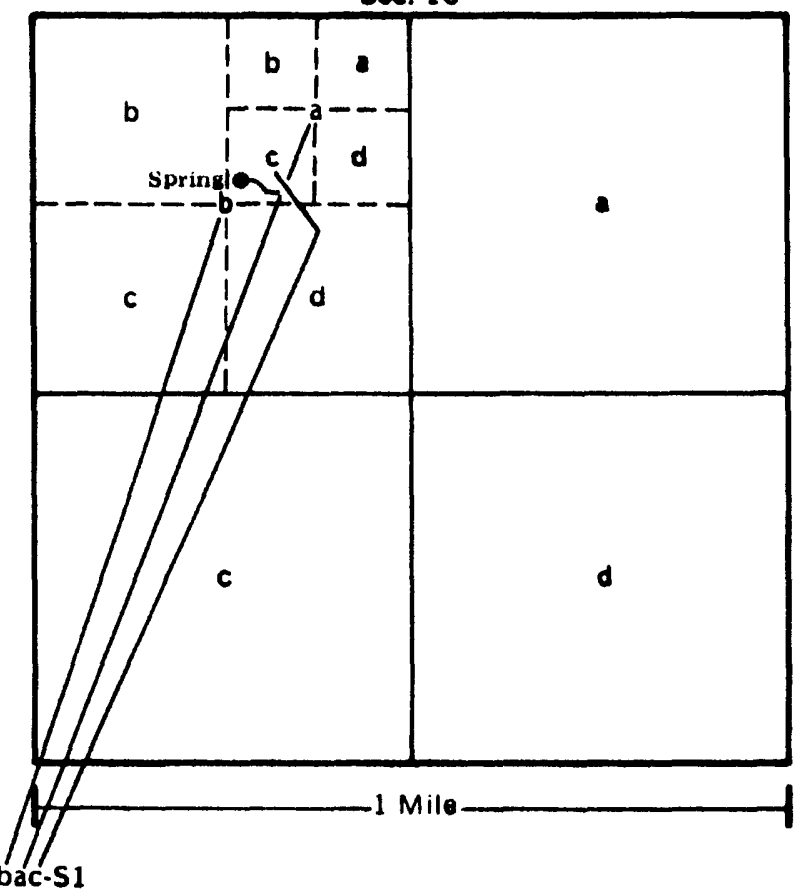

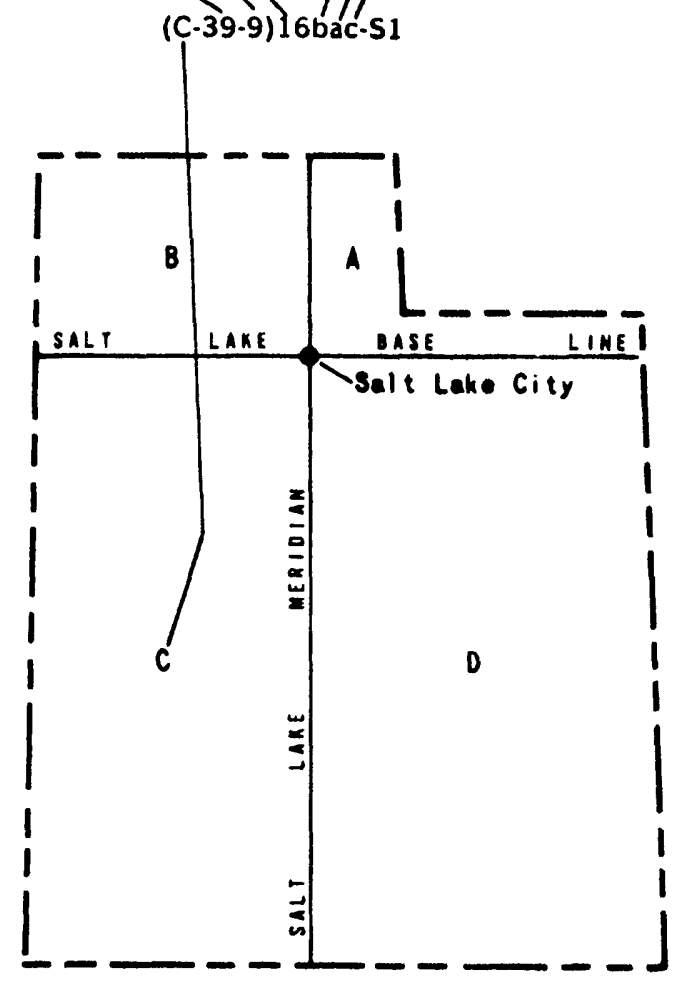

Figure 2.--Data-site numbering system used in Utah. 


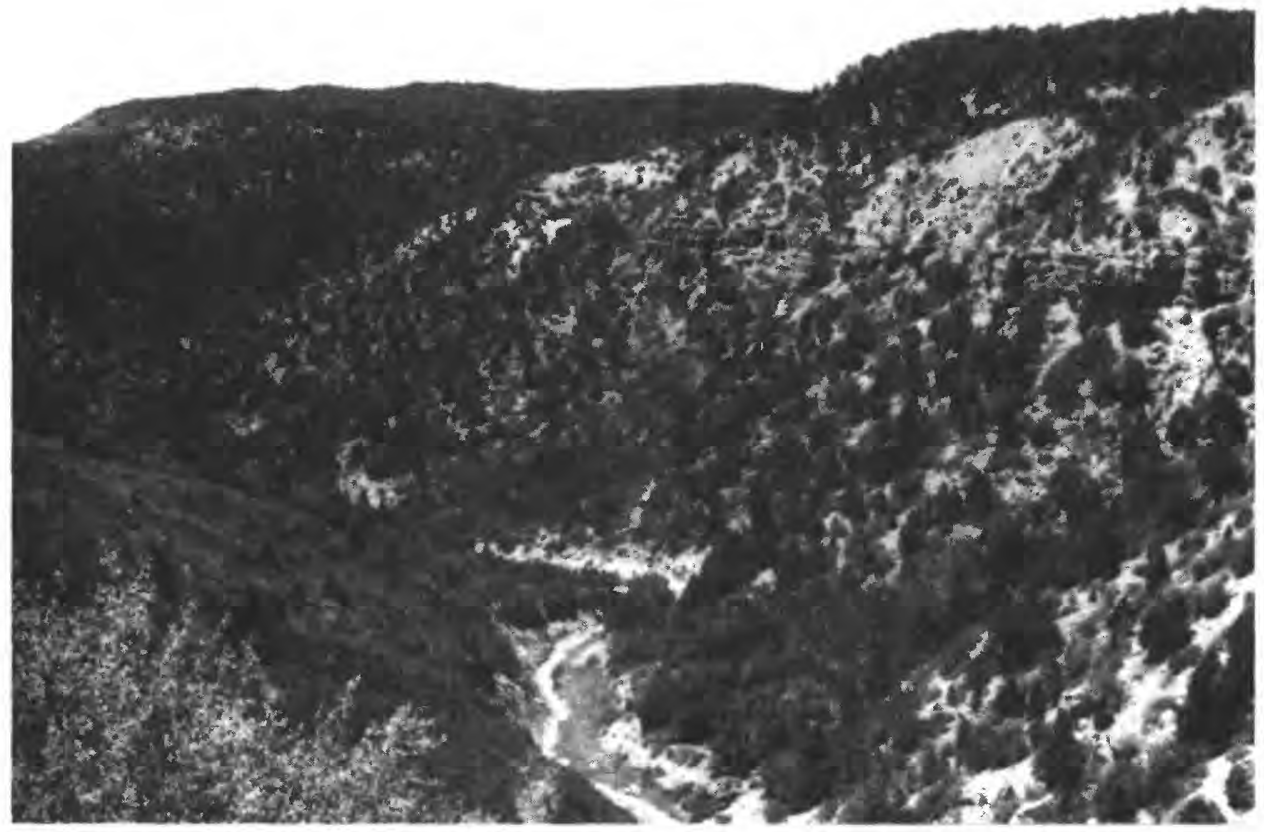

Figure 3.--Vegetation and slopes typical of the L.C. Holding coal-lease tract. View from the North Fork Road in the lease tract looking southwest down Orderville Gulch.

Summer storms commonly cause flashflooding in the narrow canyons through which the streams flow. Large sediment loads are carried by streams during these events, and massive erosion of hillsides and canyon walls also is possible. The intensity of a 1-hour storm with a recurrence interval of 2 years is nearly 1 in./hour. The intensity is 2 in./hour for a 1-hour storm with a 100-year recurrence interval (Miller and others, 1973, table 11 and figs. $19,24,25,30$ ).

The mean monthly air temperatures at higher elevations in the study area are probably similar to those at Alton $(7,040 \mathrm{ft})$; however, mean monthly temperatures at lower elevations in the western part of the study area are probably more like those at zion Canyon $(4,050 \mathrm{ft})$. Thus, the low mean monthly temperature in the area ranges from -3 to $4{ }^{\circ} \mathrm{C}$, and the high mean monthly temperature ranges from 19 to $28^{\circ} \mathrm{C}$ (fig. 4)(National Oceanic and Atmospheric Administration, 1981-86).

Annual free water-surface evaporation, an indicator of potential evaporation from natural surfaces, is about 50 in. in the study area (Farnsworth and others, 1982, maps 2, 3). About 70 to 80 percent of this evaporation ( 35 to 40 in.) occurs between May and October. 

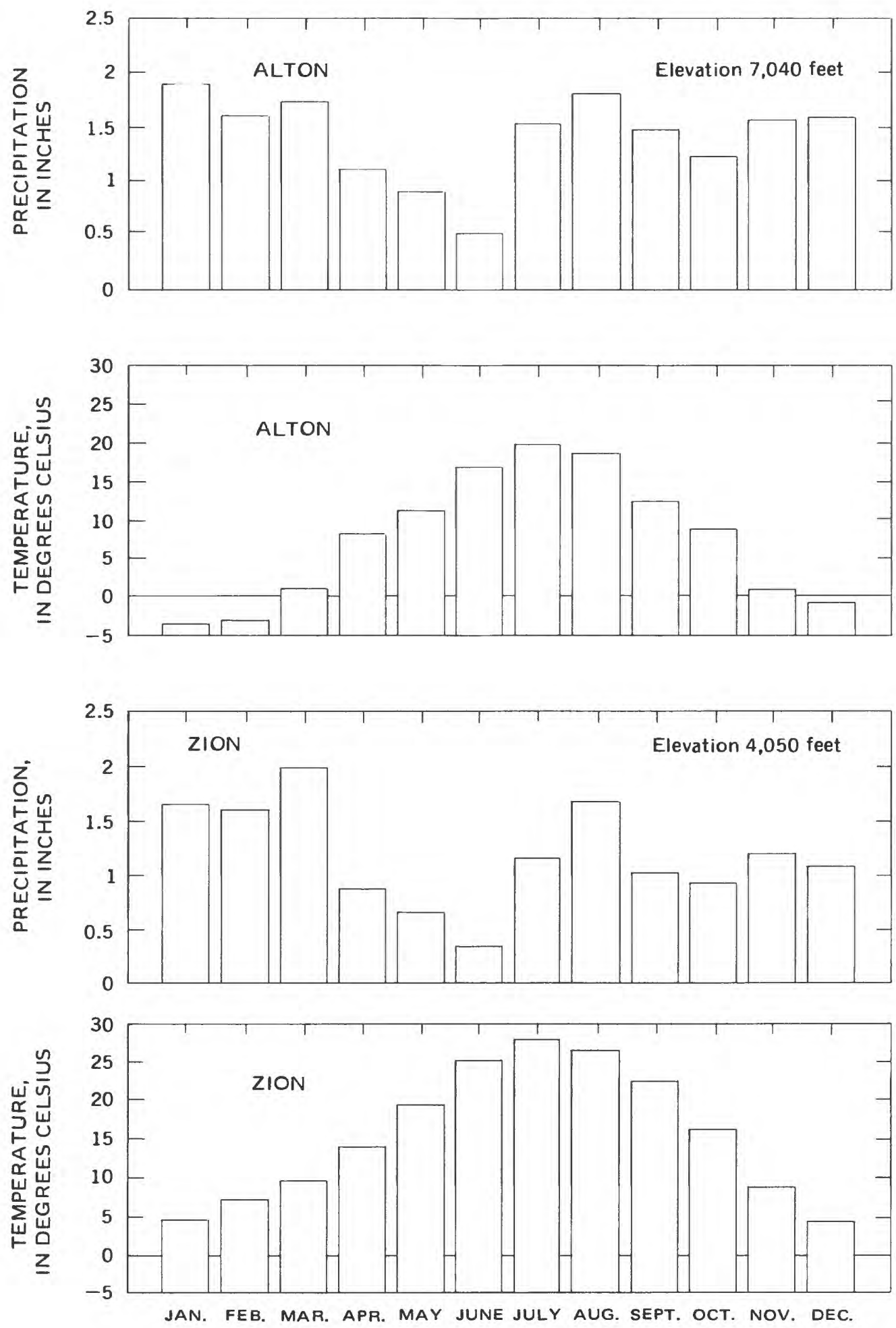

Figure 4.--Mean monthly precipitation and temperature at Alton and Zion, 1951-86. 


\section{Geology}

\section{Stratigraphy}

Geologic units exposed in the L.C. Holding coal-lease tract and the surrounding area range in age from Triassic to Holocene. They include both consolidated rocks and unconsolidated deposits. The geology of the lease tract and adjacent area is shown on plate 1.

The Upper Triassic and Lower Jurassic Navajo Sandstone is the oldest geologic unit exposed in the study area. It is a light-gray, tan, and red, fine-grained quartz sandstone with calcareous cement. The Navajo Sandstone is a massive, homogeneous sandstone that ranges from well consolidated to friable. It forms cliffs and crops out extensively to the west of the lease tract, where it forms the walls of Zion Canyon. The maximum thickness of the Navajo Sandstone is 2,280 ft at the Temple of Sinawava in Zion National Park (Gregory, 1950, p. 83). The Navajo Sandstone thins eastward; however, no information on the total thickness of the formation in the lease tract is available.

Overlying the Navajo Sandstone is the Middle Jurassic Temple Cap Sandstone. It is light-gray to tan, fine-grained, and includes crossbedded sandstone and beds of red shaly siltstone at the base (Cashion, 1967; Peterson and Pipiringos, 1979, p. B9). In the Meadow Creek area in the southeastern part of the study area shown on plate 1, the estimated thickness of the Temple Cap Sandstone is about $150 \mathrm{ft}$ according to geologic sections measured by Peterson and Pipiringos (1979, p. B10). Drilling during this study also indicated a thickness of about $150 \mathrm{ft}$ for the Temple Cap Sandstone at well LC2 (see $\log$ in Supplemental Information).

The Middle Jurassic Carmel Formation overlies the Temple Cap Sandstone and crops out within and west of the lease tract. The Carmel Formation is comprised of four members--in ascending order, they are: the limestone member, banded member, gypsiferous member, and the Winsor Member (Cashion, 1967). The limestone member, which overlies the Temple Cap Sandstone, is blue-gray to tan, silty, dense olitic to thinly bedded limestone with a thin sandy red shale at the base. Geologic sections (Cashion, 1967) show the thickness of the 1 imestone member to be about $340 \mathrm{ft}$ just west of the southwestern lease-tract boundary. Drilling during this study indicated thicknesses of about $143 \mathrm{ft} \mathrm{(well} \mathrm{LC3)} \mathrm{and} 250 \mathrm{ft}$ (well LCl) for the limestone member.

The banded member is named for its alternating beds of light-gray and reddish-brown sandstone and siltstone. This member is gypsiferous and very friable. Thickness of the banded member from geologic sections (Cashion, $1967)$ is $190 \mathrm{ft}$, and as determined from drilling, it is about $205 \mathrm{ft}$ at well LC1 (see log in Supplemental Information).

Ledge-forming, light-gray to white, massive gypsum is the main constituent of the gypsiferous member. This member is as much as $95 \mathrm{ft}$ thick in the study area (Cashion, 1967) and includes fossiliferous limestone at the top, and interbedded gypsiferous limestone, sandstone, and shale below the massive gypsum (LCl; $10 g$ in supplemental Information). Drilling during this study indicated a thickness of about $60 \mathrm{ft}$ at well $\mathrm{LCl}$. 
The Winsor Member is predominantly very fine to fine-grained, friable sandstone with some shale in the lower part (Cashion, 1967). The upper part of the member is generally light gray to yellowish gray, and the lower part is red and pink (Cashion, 1967). Geologic sections by Cashion (1967) showed a thickness of about 200 to $250 \mathrm{ft}$ for the Winsor Member; however, drilling during this study at well LCl indicated that the thickness of the Winsor Member is about $425 \mathrm{ft}$.

A major unconformity separates the Carmel Formation from the overlying Dakota Formation (Hintze, 1988, p. 193). However, Cashion (1967) has mapped the Dakota Formation and the lower coal-bearing member of the Tropic Shale as one unit; therefore, it is not shown as a separate formation on plate 1. The Dakota Formation consists of lenses of poorly consolidated conglomerate and sandstone that grade irregularly upward into sandstones, shales, and coal of the Tropic Shale (Gregory, 1950, p. 102). The average thickness of the Dakota Formation is about $40 \mathrm{ft}$ (Gregory, 1950, p. 102).

The Upper Cretaceous Tropic Shale contains the coal beds or "zones" of potential commercial value in the lease-tract area. A lower coal-bearing member and an upper shaly member form the Tropic Shale in the study area. The coal-bearing member includes brown to gray lenticular sandstone, carbonaceous shale, and coal. The coal zones of interest occur near the top and base of the coal-bearing member and consist of several coal beds each less than $4 \mathrm{ft}$ thick, interstratified with shales, sandstones, and organic material (Gregory, 1950, p. 103). The shaly member is gray, carbonaceous shale with interbedded sandstone and thin coal beds. Total thickness of the Tropic Shale, interpreted from geologic sections of the area (Cashion, 1967), is about 900 ft in the southwestern part of the area shown on plate 1, thickening eastward to about 1,100 ft west of Meadow Creek.

Overlying and intertonguing with the Tropic Shale is the Straight Cliffs Sandstone, a brown to gray, very fine grained, cliff-forming sandstone with some interbedded gray shale (Cashion, 1967). Geologic sections by Cashion (1967) indicate that the total thickness of the Straight Cliffs Sandstone is about $600 \mathrm{ft}$ in the southwestern part of the area shown on plate 1, thinning eastward to about $400 \mathrm{ft}$ west of Meadow Creek.

The Wahweap Sandstone conformably overlies the Straight Cliffs Sandstone. Although an unconformity separates the Wahweap from the overlying Kaiparowits Formation, Cashion (1961, 1967) mapped the Wahweap and Kaiparowits as a single unit because of the similarity in lithologies of the two formations. They consist of gray and brown, fine- to medium-grained sandstone with interbedded gray and red shale beds and pebble and cobble conglomerate. The basal part includes carbonaceous shale and thin noncommercial coal beds (Cashion, 1967). The total thickness of the Wahweap Sandstone and Kaiparowits Formation is variable because broad outcrops of the formations have been eroded in the study area. The total thickness ranges from about $100 \mathrm{ft}$ along the dissected plateau rims in the southern part of the lease-tract area (pl. 1) to as much as 2,000 ft along the North Fork Virgin River in the northern part of the study area. 
Formations of Tertiary age are absent in the lease tract, but crop out east (Pine Knoll) and southwest of the lease tract in the study area (pl. 1). Tertiary strata include the Wasatch Formation, a pink, red, and light-gray limestone and shale, and the remnant gravels. Quaternary units in the study area include terrace gravels along the North Fork Virgin River, extrusive rocks in the northern part of the study area, alluvium in the stream channels and flood plains of the major drainages, and landslide deposits (pl. 1).

\section{Structure}

Two major regional structural features, the Hurricane and Sevier fault zones, trend roughly north and parallel to each other. They bound the northeasterly-dipping block of which the lease tract is a part (fig. 5). The strata of this block are relatively flat-lying with an average regional dip of about $1 \frac{1}{2}$ to 2 degrees to the northeast (Gregory, 1950, p. 141). Despite the northeasterly dip, the large canyons and smaller valleys trend south or southwest as a result of fractures (Gregory, 1950, p. 158).

Surface evidence of faulting is relatively rare in the lease tract. Notable faults in the southern part of the tract trend northwest (pl. 1). Other faults north and west of the lease tract trend northeast as does one fault in the easternmost part of the lease tract. These are all normal faults, and along most faults, the downdropped side is to the north (pl. 1).

Fractures help shape the landscape of the lease tract and the surrounding area. Faults with little offset, groups of closely spaced joints, and single joints have determined the position of canyons, gulches, and valleys between buttes (Gregory, 1950, p. 158). Streams tend to follow joints that are less resistant to erosion than unfractured sandstone. This phenomenon is most obvious in Zion National Park where the dendritic tributaries of the major drainages all trend north-northwest, in opposition to the regional northeasterly dip of the strata.

These drainage patterns reflect a major joint system (fig. 5) in the Navajo Sandstone, which is exposed in Zion National Park and probably continues in the subsurface in the study area. A map of joint systems in the Park by A.J. Eardley (Hamilton, 1984, p. 40) confirms that the predominant trend is north-northwest. Hamilton (1984, p. 101) notes that the principal joint set is aligned with faults that developed in the area during the Pliocene and Pleistocene in response to extensional stresses in the region. Thus, joint formation may have coincided with faulting.

Several joint systems are apparent in exposures of the Navajo Sandstone in the Park (fig. 6); however, the most prominent joints appear to be vertical. Fracturing along bedding planes is also common as are conjugate joint systems at angles of about 60 degrees to the bedding planes (fig. 6 ). The prominent joints in the Navajo are conduits for recharge as discussed later in this report. 


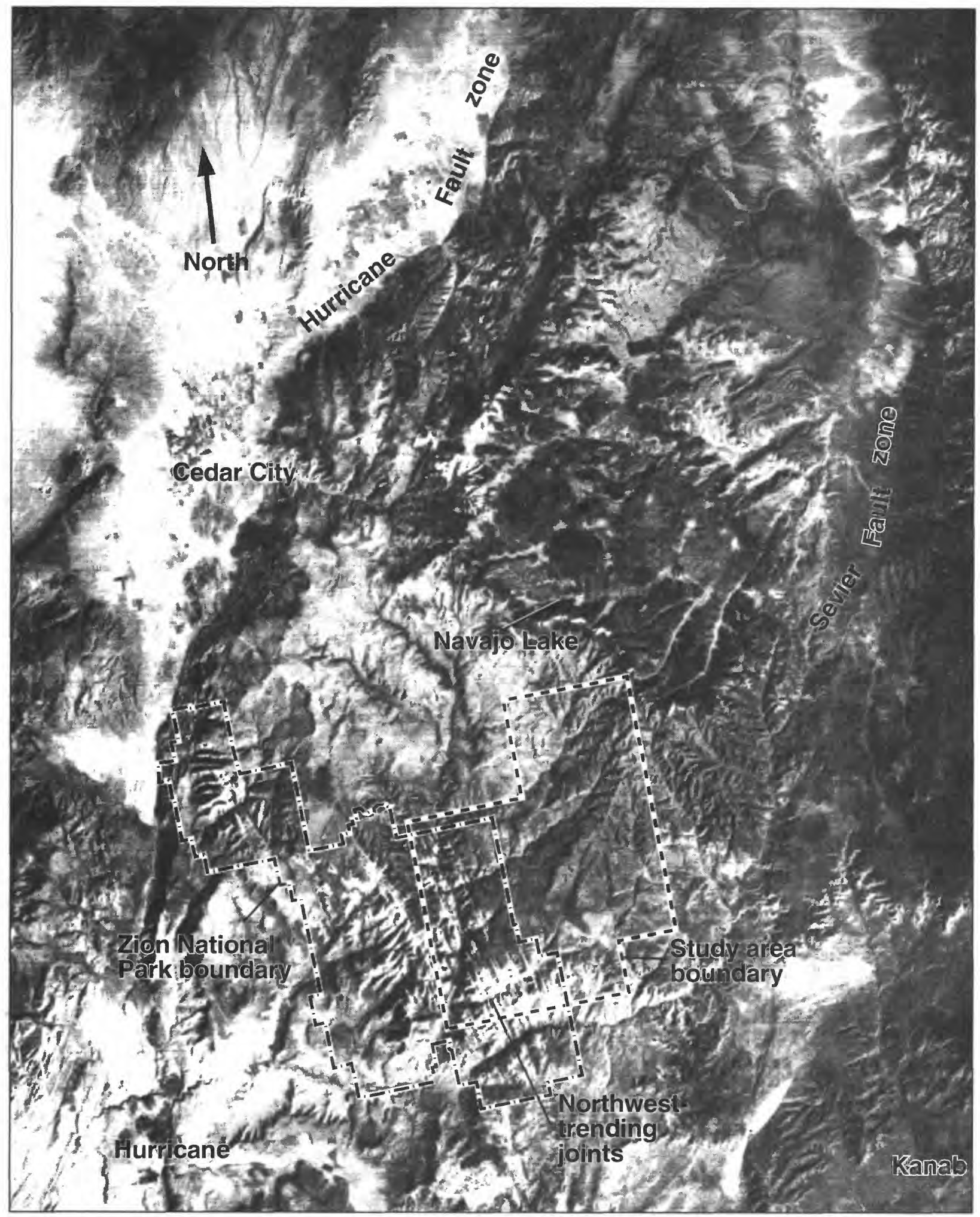

NASA ERTS E-5545-16480-5

Scale: 1 inch equals approximately 19 miles

October 15, 1976

Figure 5.--Satellite imagery showing selected features near the L.C. Holding coal-lease tract. 


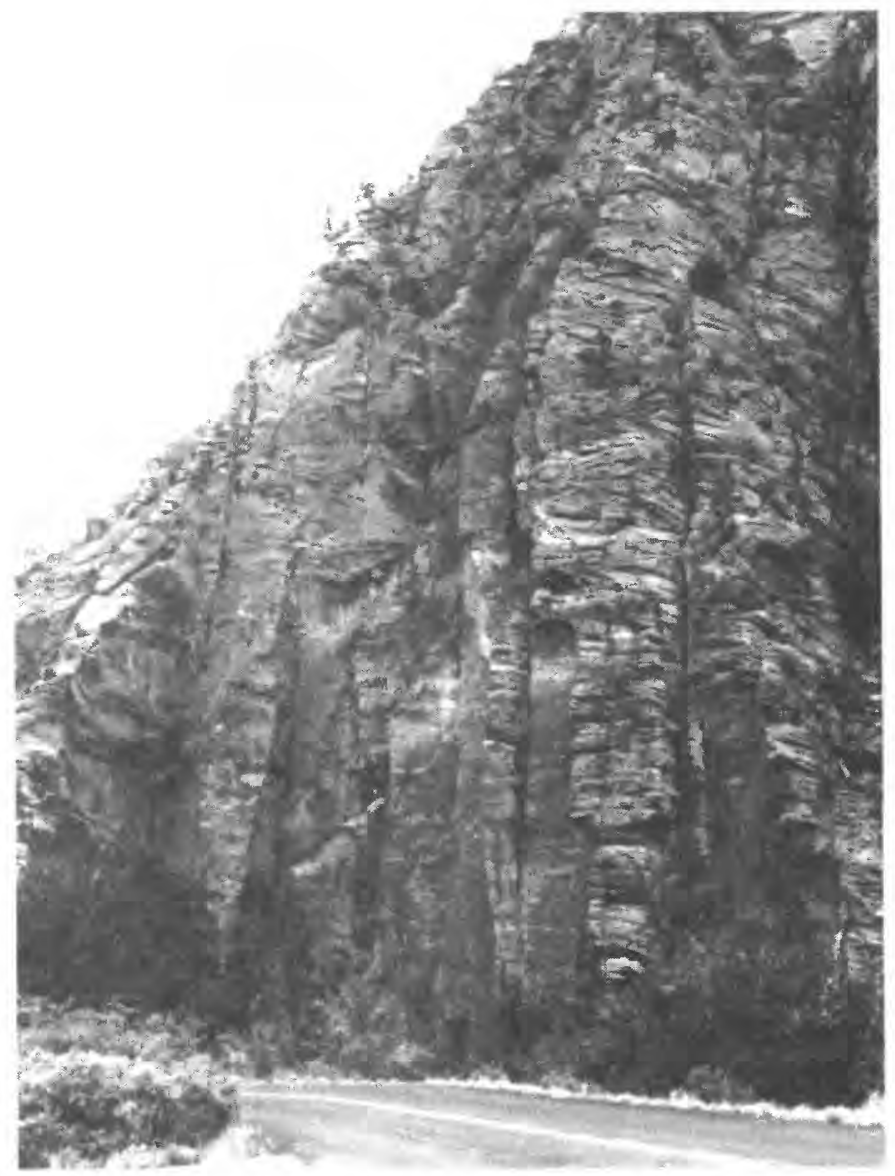

A--Major, vertical joint system. Preferred weathering along bedding planes indicated by nearly horizontal lines.

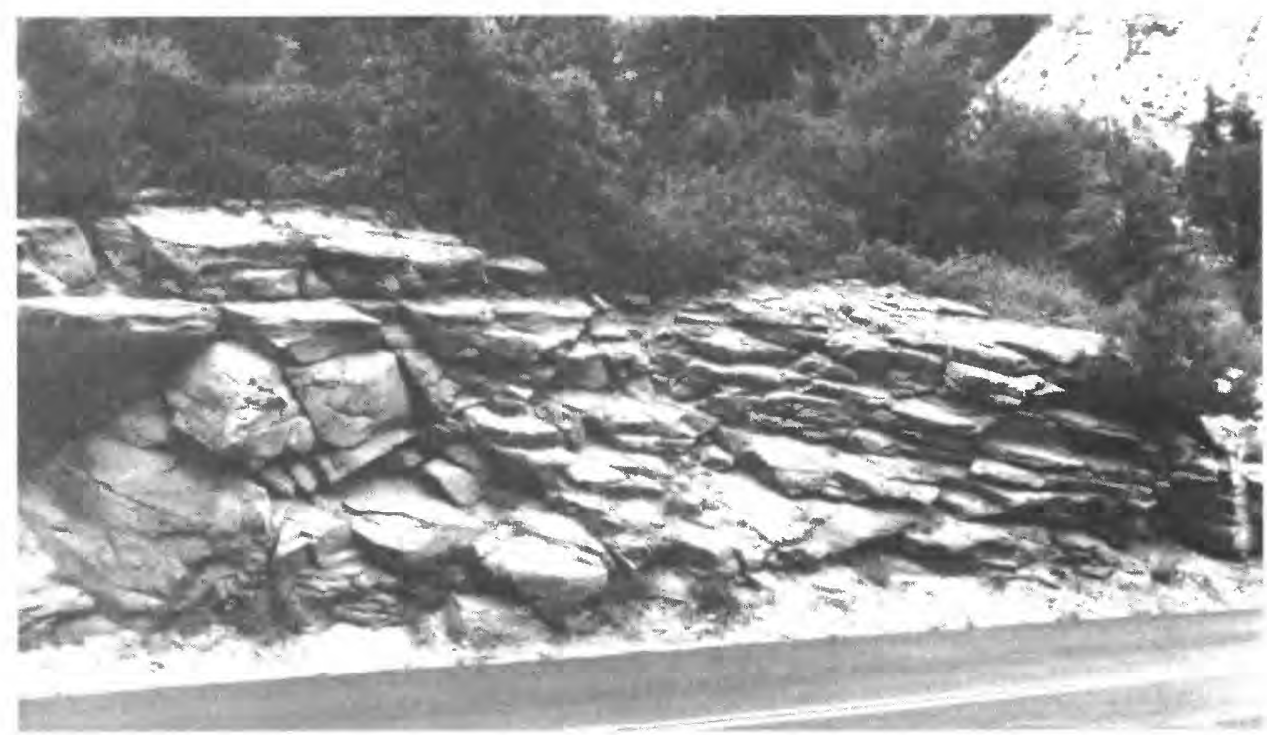

B--Bedding-plane fractures and conjugate joints at about $60^{\circ}$ to bedding.

Figure 6.--Joint structure in the Navajo Sandstone in Zion National Park: A--Major vertical joint system; and B--Bedding-plane fractures and conjugate joints at about $60^{\circ}$ to bedding. 
In most of the lease tract, the Navajo Sandstone is overlain by several hundred to several thousand feet of overburden, and the extent of joint systems is unknown. Although the overlying rocks also may be fractured, they are softer and more easily eroded than the Navajo Sandstone and thus, they are not preferentially eroded along joints (Hamilton, 1984, p. 40). However, the drainages generally trend north-south, particularly those south of the lease tract, suggesting that fracturing in these formations parallels the northnorthwest trend seen in the Navajo Sandstone.

\section{Landslide Hazards}

Erosion and sediment yield in the lease tract are particularly affected by the abundance of material from landslides and by conditions that either cause or result from other types of ground instability. Peter M. Kilbourne of the USBLM, Kanab (written cammun., 1987), mapped landslides, debris flows and slides, and slump features in the L.C. Holding coal-lease tract. These areas of ground instability are shown on plate 2. In addition, the large landslide at the southern edge of the study area has been studied in detail by Stouffer (1964).

Most of the slope failures in the study area occur in clayey strata of the Tropic Shale, particularly the basal section of the coal-bearing member (fig. 7). A bentonitic clay (shale) below the basal coal zone is probably responsible for the unstable conditions in much of the area (Stouffer, 1964, p. 1). Bentonitic clays also occur in the shaley member of the Tropic Shale. These clays swell when wet and shrink upon drying. Kilbourne (U.S. Bureau of Land Management, written camun., 1987) reports that when wet, these swelling clays are susceptible to slumping on relatively gentle slopes of as little as 8 degrees.

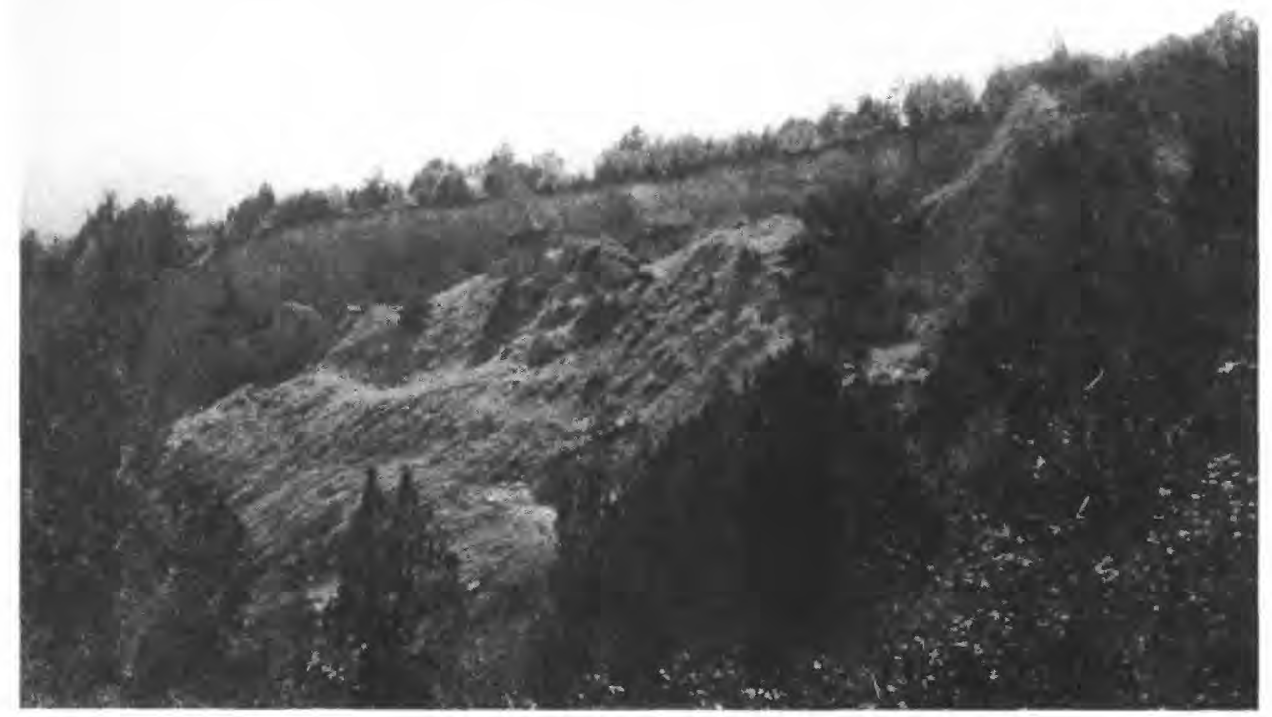

Figure 7.--Slump in coal-bearing member of the Tropic Formation near site SW 16. 
Zones of perched water in the coal-bearing member of the Tropic Shale, noted by Stouffer (1964, p. 55) in the southern part of the study area, contribute to the slope instability. Ground water reduces the shearing resistance of the bentonitic shales to the point where failure occurs (Stouffer, 1964).

Areas of unstable ground commonly occur along the steep-sided canyons adjacent to ephemeral and perennial streams as shown on plate 2. The action of the streams undercuts the steep slopes and slumping oocurs. Once disturbed by downslope movement, these unstable masses of material are easily eroded and can add large quantities of sediment to local drainages.

Steep stream banks in unconsolidated alluvial fill material are easily eroded and prone to slope failure resulting from periodic undercutting by streams. The road along Meadow Creek, which crosses alluvial fill (pl. 1), is commonly washed out when the alluvium supporting the road slumps into Meadow Creek.

Road building can cause unstable oonditions and ground movement that, in turn, can cause greater sediment yields. A road cut into material such as the Tropic Shale undercuts the slope and results in unstable conditions at the toe of the slope. In the study area, landslides and slumps are common where roads cross the Tropic Shale as in the Orderville Gulch area (pl. 2).

A landslide complex resulting from failure of bentonitic shales in the coal-bearing member of the Tropic Shale has caused problems on State Highway 9 in the Coal Hill area (pl. 2) since 1928 (Stouffer, 1964, p. 1). The landslide block, between Meadow Creek and Little Meadow Creek, is moving south. The highway was relocated to an "inactive" part of the slide in 196364, but movement has continued to cause cracking and displacement of the asphalt (fig. 8). Doelling and others (1986, p. 8) noted $0.85 \mathrm{ft}$ of horizontal offset and $0.4 \mathrm{ft}$ of vertical offset on the pavement at the western edge of the slide from the spring 1985 to May 1986.

Stouffer (1964, p. 22) attributed some slump features and collapse of overlying beds to the burning of coal beds at outcrops. In addition, he noted that in areas where the poorly consolidated beds of the banded member of the Carmel Formation crop out along stream channels, they are easily eroded and undercut, causing blocks of the overlying gypsum member to slump. This situation occurs at spring Sl4 (pl. 1), which issues from beneath a slumped block of gypsum.

Other causes of slope instability include improperly compacted construction fill noted at Birch Hollow along the North Fork road (pl. 2), oversteepened slopes in side-cast waste material noted at a contour strip mine east of the study area, and fracturing in the Straight cliffs Sandstone causing rockfalls along cliff faces (Peter M. Kilbourne, U.S. Bureau of Land Management, written commun., 1987). 


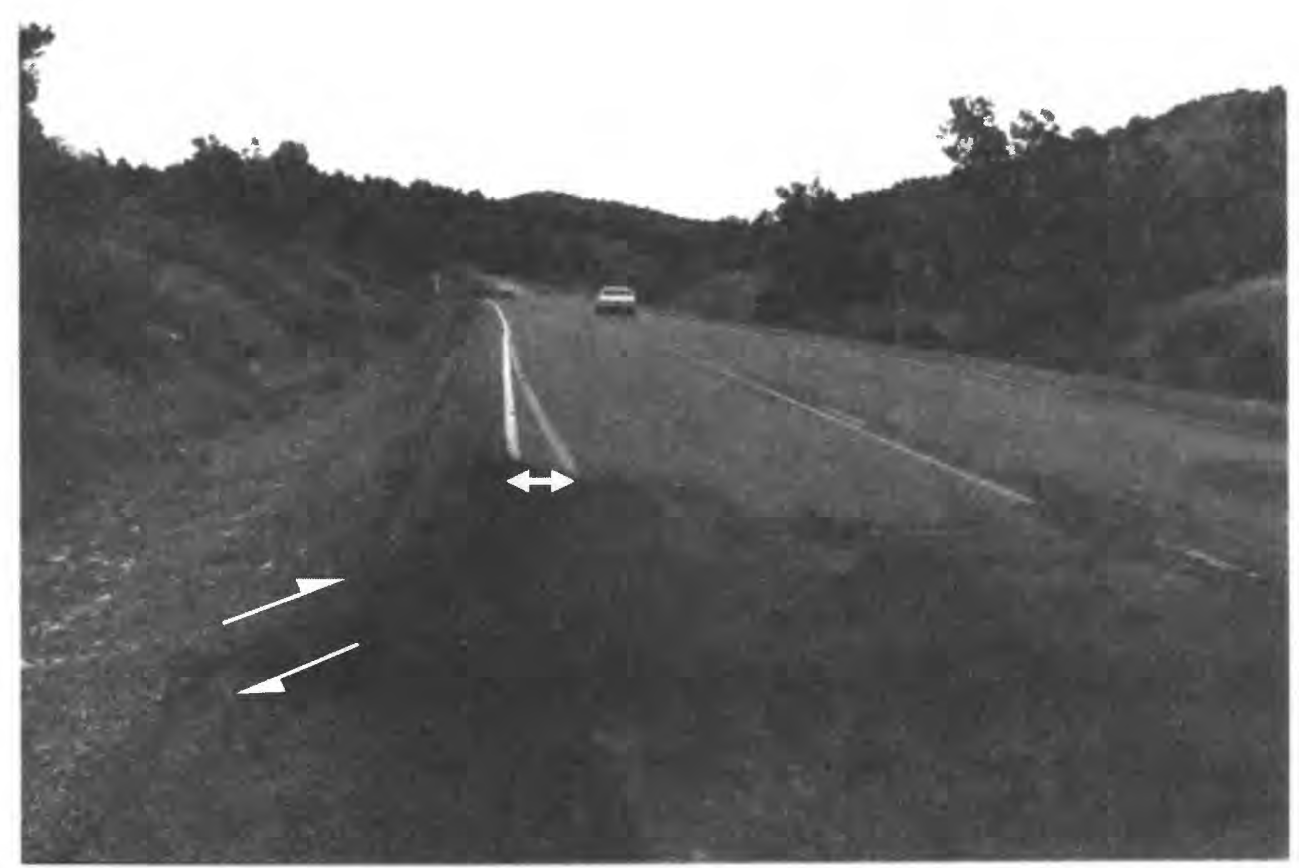

Figure 8.--Displacement of pavement by Coal Hill landslide. View looking east from western margin of slide.

\section{Land Use}

The lease tract and surrounding area are used mainly for recreation, hunting, and grazing. Summer cabins and homes are scattered throughout the area. Each year, hundreds of hikers begin the trek into Zion Canyon and "Zion Narrows" (a narrow, sheer-walled part of Zion Canyon) along the North Fork Virgin River at the northwestern margin of the lease tract. Hikers also reach zion Narrows from the western boundary of the lease tract by Orderville Gulch.

Game in the area include mule deer, turkey, mourning dove, and pigeon, and fish include trout. Hunting and fishing are popular as evidenced by the hunting club facility near Cave Canyon, in the southwestern corner of the area shown on plate 1. Local land owners graze horses, cattle, and sheep in the grassy valleys, particularly along the North Fork Virgin River.

Small-scale coal prospects have been mined during this century in the study area (Gregory, 1950, pl. 2). Small, noncomercial uranium deposits are known to occur in the Dakota Formation, 30 to $60 \mathrm{ft}$ below the lower coal zone in the Tropic Shale (U.S. Bureau of Land Management, 1986). 


\section{SURFACE-WATER HYDROLOGY}

The North Fork Virgin River drains the northern part of the lease tract, 39.5 percent of the total area. It flows southwest through the lease tract and into Zion Canyon in Zion National Park (fig. 1). The North Fork Virgin River then flows into the Virgin River about $3 \mathrm{mi}$ south of the Park's southern border. The stream in Orderville Gulch drains most of the central part of the lease tract, 36.7 percent of the total area. The headwaters of the stream in Orderville Gulch lie almost entirely within the boundaries of the lease tract (pl. 1). Clear Creek and Meadow Creek drain the southern part of the lease tract, 23.7 percent of the total area. The headwaters of clear Creek lie within the lease boundaries (pl. 1). About 0.1 percent of the lease area is drained by Corral Creek, a small tributary of Deep Creek, at the northwestern border of the study area (pl. 1). Deep Creek flows south into the North Fork Virgin River (fig. 1). Data on streamflow, temperature, specific conductance, and suspended-sediment concentration for selected streamflow sites are presented in table 1 .

\section{Streamflow Characteristics}

Runoff from snow packs and sumer thunderstorms contribute most of the flow to perennial streams (Sandberg and Sultz, 1985, p. 13). Base flow in these streams is sustained by spring discharge and diffuse seepage along the stream courses. Flow in the North Fork Virgin River originates at Cascade Spring, north of the lease tract. This spring is recharged, in part, by water from Navajo Lake and emerges from the Tertiary Wasatch Formation about 6,400 ft south of the lake (Wilson and Thomas, 1964, p. C22).

Some of the flow in the North Fork Virgin River upstream from Zion Canyon is diverted for irrigation of meadows adjacent to the river that supply grass for grazing livestock. Most of the diverted flow returns to the river as surface runoff or seepage (Sandberg and Sultz, 1985, p. 13). Perennial flows in the other drainages and tributaries are small and are not diverted. Intermittent or ephemeral streams flow into the major drainages during periods of snowmelt runoff and at times during thunderstorms, but their flows are not sustained by springs and seeps.

Perennial flow in tributaries to the North Fork Virgin River such as the streams in Hay, Rosy, Stevens, and Ashdown Canyons as well as Muddy Creek, east of the study area, begins as spring discharge from the Wasatch Formation. Other tributaries of the North Fork Virgin River such as the streams in Bulloch and Straight Canyons and Orderville Gulch (pl. 2) begin as seepage from the Wahweap Sandstone and Kaiparowits Formation.

Two U.S. Geological Survey continuous-flow stream-gaging stations were operated in the study area, but have been discontinued. A station (09405420) on the North Fork Virgin River downstream fram Bulloch Canyon (SW2, pl . 2) provided continuous records from October 1974 through September 1984. About 3 mi downstream, a second station (09405450), located in the lease tract on the North Fork Virgin River above Zion Narrows (SW4, pl. 2), provided continuous records from October 1978 through September 1984. Long-term continuous records are available for the North Fork Virgin River at an active U.S. Geological Survey gaging station (09405500) near the Visitor Center in zion National Park (ZSwlo, fig. 9). 
Table 1.--Streamflow, temperature, specific conductance, and suspended-sediment concentration for selected streamflow sites

$\left[\mathrm{ft}^{3} / \mathrm{s}\right.$, aubic feet per second; ${ }^{\circ} \mathrm{C}$, degrees Celsius; $\mu \delta / \mathrm{cm}$, microsiemens per cent imeter at 25 degrees Celsius; $\mathrm{mg} / \mathrm{h}$, milligrams per liter]

Site identifier: Locations shown on plate 2 or figure 9.

Streamflow: E, est imated.

Suspended-sediment concentration: (4), Value is average of number of values shown in parentheses.

\begin{tabular}{|c|c|c|c|c|c|c|}
\hline $\begin{array}{c}\text { Site } \\
\text { ident ifier }\end{array}$ & Stream & Date & $\begin{array}{l}\text { Stream- } \\
\text { flow, } \\
\text { instan- } \\
\text { taneous } \\
\left(\mathrm{ft}^{3} / \mathrm{s}\right)\end{array}$ & $\begin{array}{l}\text { Temper- } \\
\text { ature } \\
\left({ }^{\circ} \mathrm{C}\right)\end{array}$ & $\begin{array}{l}\text { Spe- } \\
\text { cific } \\
\text { con- } \\
\text { duct- } \\
\text { ance } \\
(\mu S / \mathrm{cm})\end{array}$ & $\begin{array}{l}\text { Sus- } \\
\text { pended- } \\
\text { sedi- } \\
\text { ment } \\
\text { concen- } \\
\text { tration } \\
\text { (mg } / \mathrm{L})\end{array}$ \\
\hline
\end{tabular}

\section{VIRGIN RIVER DRAINAGE}

SWI North Fork Virgin River below Rosy Canyon

SWR North Fork Virgin River below Bulloch Canyon

\begin{tabular}{|c|c|c|c|c|}
\hline $\begin{array}{l}06-11-86 \\
07-16-86 \\
11-05-86 \\
10-26-74 \\
12-04-74\end{array}$ & $\begin{array}{l}13 \\
13 \\
6.8 \\
8.7 \\
8.6\end{array}$ & $\begin{array}{r}11.0 \\
14.0 \\
5.5 \\
-\overline{2.0}\end{array}$ & $\begin{array}{l}345 \\
390 \\
425 \\
-- \\
435\end{array}$ & $\begin{array}{c}18 \\
36 \\
2,160 \\
--\end{array}$ \\
\hline $\begin{array}{l}03-20-75 \\
04-23-75 \\
05-09-75 \\
05-19-75\end{array}$ & $\begin{array}{l}14 \\
20 \\
10 \\
18\end{array}$ & $\begin{array}{r}7.5 \\
12.5 \\
12.5\end{array}$ & $\begin{array}{l}-- \\
440 \\
480\end{array}$ & $\begin{array}{r}221 \\
579 \\
3 \overline{3} 9\end{array}$ \\
\hline $\begin{array}{l}06-16-75 \\
07-22-75 \\
08-21-75 \\
10-08-75 \\
11-10-75\end{array}$ & $\begin{array}{l}20 \\
14 \\
14 \\
7.3 \\
6.0\end{array}$ & $\begin{array}{r}16.0 \\
17.0 \\
-- \\
7.0 \\
6.0\end{array}$ & $\begin{array}{l}360 \\
375 \\
-- \\
395 \\
420\end{array}$ & $\begin{array}{r}- \\
31,300 \\
- \\
-\end{array}$ \\
\hline $\begin{array}{l}02-03-76 \\
03-18-76 \\
05-04-76 \\
06-09-76 \\
07-13-76\end{array}$ & $\begin{array}{l}8.3 \\
16 \\
11 \\
15 \\
11\end{array}$ & $\begin{array}{r}2.0 \\
8.0 \\
7.0 \\
14.0 \\
15.0\end{array}$ & $\begin{array}{l}420 \\
430 \\
440 \\
400 \\
390\end{array}$ & $\begin{array}{l}- \\
-- \\
- \\
-\end{array}$ \\
\hline $\begin{array}{l}07-13-76 \\
08-03-76 \\
09-21-76 \\
10-02-76 \\
10-18-77\end{array}$ & $\begin{array}{l}30 \\
9.0 \\
11 \\
28 \\
5.1\end{array}$ & $\begin{array}{r}14 . \overline{5} \\
-- \\
11 . \overline{0}\end{array}$ & $\begin{array}{l}380 \\
-- \\
440\end{array}$ & $\begin{array}{r}18,100 \\
-- \\
1,160 \\
33,800 \\
--\end{array}$ \\
\hline $\begin{array}{l}02-22-78 \\
04-24-78 \\
06-08-78 \\
06-28-78 \\
08-08-78\end{array}$ & $\begin{array}{l}12 \\
46 \\
29 \\
23 \\
12\end{array}$ & $\begin{array}{l}-\overline{0} \\
12.0 \\
12.0 \\
15.0 \\
16.5\end{array}$ & $\begin{array}{l}430 \\
430 \\
350 \\
300 \\
390\end{array}$ & $\begin{array}{l}- \\
- \\
- \\
-\end{array}$ \\
\hline $\begin{array}{l}09-07-78 \\
10-13-78 \\
12-07-78 \\
04-26-79 \\
05-17-79\end{array}$ & $\begin{array}{l}13 \\
14 \\
6.6 \\
68 \\
58\end{array}$ & $\begin{array}{r}15.0 \\
-- \\
0.0 \\
9.0 \\
10.5\end{array}$ & $\begin{array}{l}360 \\
335 \\
450 \\
550 \\
430\end{array}$ & $\begin{array}{l}- \\
-- \\
- \\
-\end{array}$ \\
\hline $\begin{array}{l}06-14-79 \\
07-16-79 \\
08-21-79 \\
10-01-79 \\
10-31-79\end{array}$ & $\begin{array}{l}34 \\
16 \\
21 \\
16 \\
18\end{array}$ & $\begin{array}{r}15.0 \\
-. \\
16.0 \\
15.5 \\
8.0\end{array}$ & $\begin{array}{l}390 \\
370 \\
385 \\
350 \\
360\end{array}$ & $\begin{array}{l}- \\
- \\
- \\
-\end{array}$ \\
\hline
\end{tabular}


Table 1.--Streamflow, temperature, specific conductance, and suspendedsediment concentration for selected streamflow sites--Continued

\begin{tabular}{|c|c|c|c|c|c|c|}
\hline $\begin{array}{c}\text { Site } \\
\text { identifier }\end{array}$ & Stream & Date & $\begin{array}{l}\text { Stream- } \\
\text { flow, } \\
\text { instan- } \\
\text { taneous } \\
\left(\mathrm{ft}^{3} / \mathrm{s}\right)\end{array}$ & $\begin{array}{l}\text { Temper- } \\
\text { ature } \\
\left({ }^{\circ} \mathrm{C}\right)\end{array}$ & $\begin{array}{l}\text { Spe- } \\
\text { cific } \\
\text { con- } \\
\text { duct- } \\
\text { ance } \\
(\mu S / \mathrm{cm})\end{array}$ & $\begin{array}{l}\text { Sus- } \\
\text { pended- } \\
\text { sedi- } \\
\text { ment } \\
\text { concen- } \\
\text { tration } \\
\text { (mg/L) }\end{array}$ \\
\hline \multirow[t]{9}{*}{ SWR } & North Fork Virgin River below Bulloch Canyon & $\begin{array}{l}12-04-79 \\
04-16-80 \\
05-28-80 \\
07-08-80 \\
10-29-80\end{array}$ & $\begin{array}{l}18 \\
54 \\
70 \\
34 \\
19\end{array}$ & $\begin{array}{r}3.5 \\
3.0 \\
8.5 \\
-- \\
4.0\end{array}$ & $\begin{array}{l}390 \\
480 \\
420 \\
430 \\
395\end{array}$ & $\begin{array}{l}- \\
\overline{-} \\
\overline{-}\end{array}$ \\
\hline & & $\begin{array}{l}12-01-80 \\
01-13-81 \\
03-19-81 \\
04-09-81 \\
06-02-81\end{array}$ & $\begin{array}{l}20 \\
18 \\
16 \\
18 \\
21\end{array}$ & $\begin{array}{l}5.0 \\
2.5 \\
3.0 \\
5.0 \\
9.0\end{array}$ & $\begin{array}{l}370 \\
375 \\
460 \\
450 \\
400\end{array}$ & $\begin{array}{l}- \\
-- \\
-- \\
--\end{array}$ \\
\hline & & $\begin{array}{l}07-08-81 \\
08-05-81 \\
08-20-81 \\
09-10-81 \\
10-07-81\end{array}$ & $\begin{array}{l}12 \\
9.2 \\
9.2 \\
9.3 \\
9.7\end{array}$ & $\begin{array}{r}19.0 \\
9.0 \\
14.5 \\
-\overline{8.0}\end{array}$ & $\begin{array}{l}365 \\
405 \\
385 \\
405 \\
430\end{array}$ & $\begin{array}{l}- \\
-- \\
- \\
-\end{array}$ \\
\hline & & $\begin{array}{l}11-10-81 \\
12-15-81 \\
02-19-82 \\
04-15-82 \\
05-03-82\end{array}$ & $\begin{array}{l}11 \\
12 \\
11 \\
31 \\
42\end{array}$ & $\begin{array}{r}9.0 \\
5.0 \\
1.0 \\
8.0 \\
12.0\end{array}$ & $\begin{array}{l}410 \\
485 \\
455 \\
480 \\
435\end{array}$ & $\begin{array}{l}-- \\
-- \\
-- \\
--\end{array}$ \\
\hline & & $\begin{array}{l}05-14-82 \\
06-17-82 \\
07-29-82 \\
09-08-82 \\
10-04-82\end{array}$ & $\begin{array}{l}24 \\
20 \\
16 \\
15 \\
19\end{array}$ & $\begin{array}{r}9.0 \\
13.0 \\
12.5 \\
11.5 \\
5.5\end{array}$ & $\begin{array}{l}420 \\
450 \\
430 \\
420 \\
410\end{array}$ & $\begin{array}{l}- \\
-- \\
- \\
-\end{array}$ \\
\hline & & $\begin{array}{l}11-16-82 \\
02-01-83 \\
04-26-83 \\
05-19-83 \\
06-15-83\end{array}$ & $\begin{array}{l}13 \\
14 \\
67 \\
85 \\
52\end{array}$ & $\begin{array}{r}3.0 \\
0.5 \\
5.5 \\
6.0 \\
15.0\end{array}$ & $\begin{array}{l}455 \\
475 \\
490 \\
450 \\
400\end{array}$ & $\begin{array}{l}- \\
- \\
- \\
-\end{array}$ \\
\hline & & $\begin{array}{l}07-18-83 \\
08-23-83 \\
10-13-83 \\
02-09-84 \\
03-22-84\end{array}$ & $\begin{array}{l}21 \\
27 \\
22 \\
25 \\
22\end{array}$ & $\begin{array}{r}12.0 \\
11.0 \\
9.0 \\
3.0 \\
3.0\end{array}$ & $\begin{array}{l}-- \\
400 \\
395 \\
395 \\
375\end{array}$ & $\begin{array}{l}-- \\
-- \\
- \\
-\end{array}$ \\
\hline & & $\begin{array}{l}04-16-84 \\
05-23-84 \\
06-14-84 \\
07-16-84 \\
08-16-84\end{array}$ & $\begin{array}{l}20 \\
20 \\
17 \\
16 \\
22\end{array}$ & $\begin{array}{r}9.0 \\
15.5 \\
15.0 \\
13.0 \\
18.0\end{array}$ & $\begin{array}{l}405 \\
400 \\
390 \\
400 \\
380\end{array}$ & $\begin{array}{l}-- \\
- \\
-- \\
-\end{array}$ \\
\hline & & $\begin{array}{l}09-13-84 \\
10-03-84 \\
06-12-86 \\
11-05-86\end{array}$ & $\begin{array}{l}9.6 \\
12 \\
19 \\
11\end{array}$ & $\begin{array}{r}10.0 \\
10.0 \\
16.5 \\
8.5\end{array}$ & $\begin{array}{l}430 \\
435 \\
360 \\
410\end{array}$ & $\overline{-}$ \\
\hline SWB & North Fork Virgin River near Willow Canyon & $\begin{array}{l}06-18-86 \\
11-05-86\end{array}$ & $\begin{array}{l}17 \\
12\end{array}$ & $\begin{array}{r}20.0 \\
9.5\end{array}$ & $\begin{array}{l}385 \\
470\end{array}$ & $13(4)$ \\
\hline SW4 & North Fork Virgin River above Zion Narrows & $\begin{array}{l}04-26-79 \\
05-17-79 \\
06-14-79 \\
07-16-79 \\
0\end{array}$ & $\begin{array}{l}59 \\
56 \\
38 \\
18\end{array}$ & $\begin{array}{r}9.5 \\
11.0 \\
15.5 \\
20.0\end{array}$ & $\begin{array}{l}560 \\
440 \\
400 \\
365\end{array}$ & $\begin{array}{l}- \\
-- \\
-\end{array}$ \\
\hline
\end{tabular}


Table 1.--Streamflow, temperature, specific conductance, and suspendedsediment concentration for selected streamflow sites--Continued

\begin{tabular}{|c|c|c|c|c|c|c|}
\hline $\begin{array}{c}\text { Site } \\
\text { ident ifier }\end{array}$ & Stream & Date & $\begin{array}{l}\text { Stream- } \\
\text { flow, } \\
\text { instan- } \\
\text { taneous } \\
\left(\mathrm{ft}^{3} / \mathrm{s}\right)\end{array}$ & $\begin{array}{l}\text { Temper- } \\
\text { ature } \\
\left({ }^{\circ} \mathrm{C}\right)\end{array}$ & $\begin{array}{l}\text { Spe- } \\
\text { cific } \\
\text { con- } \\
\text { duct- } \\
\text { ance } \\
(\mu S / \mathrm{cm})\end{array}$ & $\begin{array}{l}\text { Sus- } \\
\text { pended- } \\
\text { sedi- } \\
\text { ment } \\
\text { concen- } \\
\text { tration } \\
\text { (mg/L) }\end{array}$ \\
\hline \multirow[t]{9}{*}{ SWA } & North Fork Virgin River above Zion Narrows & $\begin{array}{l}08-21-79 \\
10-01-79 \\
10-31-79 \\
12-04-79 \\
04-16-80\end{array}$ & $\begin{array}{l}23 \\
16 \\
17 \\
17 \\
66\end{array}$ & $\begin{array}{r}15.5 \\
13.0 \\
8.0 \\
4.0 \\
--\end{array}$ & $\begin{array}{l}405 \\
375 \\
365 \\
390 \\
560\end{array}$ & $\begin{array}{l}- \\
- \\
- \\
-\end{array}$ \\
\hline & & $\begin{array}{l}05-28-80 \\
07-08-80 \\
08-22-80 \\
10-\not 2-80 \\
12-01-80\end{array}$ & $\begin{array}{l}66 \\
24 \\
19 \\
19 \\
19\end{array}$ & $\begin{array}{r}11.0 \\
-- \\
14.0 \\
4.0 \\
1.5\end{array}$ & $\begin{array}{r}465 \\
165 \\
420 \\
445 \\
--\end{array}$ & $\begin{array}{l}-- \\
\overline{-} \\
-\end{array}$ \\
\hline & & $\begin{array}{l}01-13-81 \\
03-19-81 \\
04-09-81 \\
04-22-81 \\
05-05-81\end{array}$ & $\begin{array}{l}18 \\
17 \\
17 \\
27 \\
15\end{array}$ & $\begin{array}{r}3.5 \\
4.0 \\
7.0 \\
11.0 \\
9.5\end{array}$ & $\begin{array}{l}440 \\
550 \\
520 \\
520 \\
500\end{array}$ & $\overline{-}$ \\
\hline & & $\begin{array}{l}06-02-81 \\
07-08-81 \\
08-05-81 \\
08-20-81 \\
08-26-81\end{array}$ & $\begin{array}{r}18 \\
12 \\
6.3 \\
7.5 \\
6.9\end{array}$ & $\begin{array}{r}9.5 \\
16.5 \\
9.0 \\
16.0 \\
16.5\end{array}$ & $\begin{array}{l}445 \\
420 \\
430 \\
420 \\
415\end{array}$ & $\begin{array}{l}-- \\
-- \\
-- \\
66\end{array}$ \\
\hline & & $\begin{array}{l}09-10-81 \\
10-07-81 \\
10-08-81 \\
11-10-81 \\
12-15-81\end{array}$ & $\begin{array}{c}7.7 \\
8.6 \\
7.3 \\
10 \\
9.8\end{array}$ & $\begin{array}{r}-- \\
10.0 \\
14.5 \\
8.0 \\
4.0\end{array}$ & $\begin{array}{l}430 \\
465 \\
435 \\
465 \\
540\end{array}$ & $\begin{array}{l}-- \\
-- \\
-- \\
-\end{array}$ \\
\hline & & $\begin{array}{l}02-19-82 \\
04-15-82 \\
04-21-82 \\
05-03-82 \\
05-14-82\end{array}$ & $\begin{array}{l}12 \\
29 \\
22 \\
34 \\
23\end{array}$ & $\begin{array}{r}1.0 \\
10.5 \\
6.5 \\
14.5 \\
13.0\end{array}$ & $\begin{array}{l}540 \\
530 \\
560 \\
455 \\
465\end{array}$ & $\begin{array}{l}-- \\
- \\
- \\
-\end{array}$ \\
\hline & & $\begin{array}{l}06-17-82 \\
07-29-82 \\
08-23-82 \\
09-08-82 \\
10-04-82\end{array}$ & $\begin{array}{c}18 \\
12 \\
13 \\
9.9 \\
16\end{array}$ & $\begin{array}{r}13.0 \\
18.0 \\
22.0 \\
12.5 \\
8.0\end{array}$ & $\begin{array}{l}500 \\
425 \\
360 \\
450 \\
430\end{array}$ & $\begin{array}{l}- \\
-- \\
- \\
-\end{array}$ \\
\hline & & $\begin{array}{l}11-16-82 \\
02-01-83 \\
04-26-83 \\
06-15-83\end{array}$ & $\begin{array}{c}13 \\
6.3 \\
74 \\
58\end{array}$ & $\begin{array}{r}3.0 \\
0.5 \\
6.5 \\
15.0\end{array}$ & $\begin{array}{l}540 \\
620 \\
550 \\
445\end{array}$ & $\begin{array}{l}-- \\
- \\
--\end{array}$ \\
\hline & & $\begin{array}{l}07-18-83 \\
08-23-83 \\
06-18-86 \\
07-16-86 \\
11-05-86\end{array}$ & $\begin{array}{l}24 \\
26 \\
18 \\
17 \\
11\end{array}$ & $\begin{array}{r}12.0 \\
15.0 \\
14.5 \\
20.0 \\
9.0\end{array}$ & $\begin{array}{l}415 \\
430 \\
430 \\
410 \\
455\end{array}$ & $\begin{array}{l}\overline{-} \\
\overline{13} \\
246 \\
--\end{array}$ \\
\hline
\end{tabular}


Table 1.--Streamflow, temperature, specific conductance, and suspendedsediment concentration for selected streamflow sites--Continued

\begin{tabular}{|c|c|c|c|c|c|c|}
\hline $\begin{array}{c}\text { Site } \\
\text { identifier }\end{array}$ & Stream & Date & $\begin{array}{l}\text { Stream- } \\
\text { flow, } \\
\text { instan- } \\
\text { taneous } \\
\left(\mathrm{ft}^{3} / \mathrm{s}\right)\end{array}$ & $\begin{array}{l}\text { Temper- } \\
\text { ature } \\
\left({ }^{\circ} \mathrm{C}\right)\end{array}$ & $\begin{array}{l}\text { Spe- } \\
\text { cific } \\
\text { con- } \\
\text { duct- } \\
\text { ance } \\
(\mu \mathrm{S} / \mathrm{cm})\end{array}$ & $\begin{array}{l}\text { Sus- } \\
\text { pended- } \\
\text { sedi- } \\
\text { ment } \\
\text { concen- } \\
\text { tration } \\
\text { (mg/L) }\end{array}$ \\
\hline $\begin{array}{l}\text { SW5 } \\
\text { SW6 } \\
\text { SW7 } \\
\text { SW8 } \\
\text { SW9 } \\
\text { SW10 }\end{array}$ & $\begin{array}{l}\text { North Fork Virgin River above Bulloch's cabin } \\
\text { North Fork Virgin River below Bulloch's cabin } \\
\text { Little Hopp Canyon at mouth } \\
\text { Bulloch Canyon at mouth } \\
\text { Hay Canyon (site 1) } \\
\text { Hay Canyon (site 2) }\end{array}$ & $\begin{array}{l}07-29-87 \\
09-18-87 \\
11-05-86 \\
11-05-86 \\
06-13-86 \\
06-13-86\end{array}$ & $\begin{array}{r}- \\
6.2 \\
0.05 \\
3.5 \\
0.16 \\
0.09\end{array}$ & $\begin{array}{r}20.0 \\
4.5 \\
0.5 \\
16.5 \\
23.0\end{array}$ & $\begin{array}{l}395 \\
425 \\
590 \\
420 \\
500 \\
460\end{array}$ & $\begin{array}{l}-- \\
- \\
= \\
- \\
-\end{array}$ \\
\hline $\begin{array}{l}\operatorname{SW11} \\
\operatorname{SW12} \\
\operatorname{SW13}\end{array}$ & $\begin{array}{l}\text { Hay Canyon (site } 3 \text { ) } \\
\text { Hay Canyon above Millet Canyon } \\
\text { Hay Canyon below Millet Canyon }\end{array}$ & $\begin{array}{l}06-13-86 \\
08-25-86 \\
06-13-86 \\
07-16-86 \\
11-05-86\end{array}$ & $\begin{array}{l}0.03 \\
0.04 \\
0.06 \\
0.14 \\
0.22\end{array}$ & $\begin{array}{r}23.0 \\
14.0 \\
28.5 \\
24.0 \\
8.0\end{array}$ & $\begin{array}{r}450 \\
1,140 \\
1,390 \\
1,360 \\
1,160\end{array}$ & $\begin{array}{r}-\overline{5} \\
5 \\
18 \\
--\end{array}$ \\
\hline $\begin{array}{l}\text { SW14 } \\
\text { SW15 } \\
\text { SW16 }\end{array}$ & $\begin{array}{l}\text { Millet Canyon at mouth } \\
\text { Tributary to Willow Canyon } \\
\text { Unnamed tributary to North Fork Virgin River }\end{array}$ & $\begin{array}{l}08-24-86 \\
07-17-86 \\
09-12-86 \\
09-18-87\end{array}$ & $\begin{array}{l}0.008 \\
0.016 \\
0.07\end{array}$ & $\begin{array}{l}16.0 \\
17.0 \\
17.5 \\
19.0\end{array}$ & $\begin{array}{r}1,450 \\
1,250 \\
980 \\
910\end{array}$ & $\frac{18}{2}$ \\
\hline \multirow[t]{2}{*}{$\sin 7$} & $\begin{array}{l}\text { Right fork unnamed tributary to North Fork } \\
\text { Virgin River }\end{array}$ & $09-11-86$ & 0.09 & 14.5 & 520 & - \\
\hline & \multicolumn{6}{|c|}{ ORDERVILLE GULCH DRAINAGE } \\
\hline $\begin{array}{l}\text { SW18 } \\
\text { SW19 }\end{array}$ & $\begin{array}{l}\text { Orderville Gulch above Right Fork } \\
\text { Orderville Gulch below Twins Hollow } \\
\text { Orderville Gulch above roadcrossing }\end{array}$ & $\begin{array}{l}06-23-86 \\
06-22-86 \\
09-09-86 \\
11-06-86 \\
06-22-86\end{array}$ & $\begin{array}{l}0.010 \\
0.04 \\
0.05 \\
0.09 \\
0.25\end{array}$ & $\begin{array}{l}18.0 \\
22.5 \\
16.0 \\
3.5 \\
26.5\end{array}$ & $\begin{array}{l}720 \\
990 \\
990 \\
910 \\
650\end{array}$ & $\begin{array}{r}\overline{20} \\
767 \\
- \\
-\end{array}$ \\
\hline SW21 & Orderville Gulch at roadcrossing & $\begin{array}{l}09-09-86 \\
11-06-86 \\
08-20-81 \\
05-03-82 \\
06-21-86\end{array}$ & $\begin{array}{l}0.20 \\
0.39 \\
0.7 \\
0.5 \\
0.24\end{array}$ & $\begin{array}{r}19.0 \\
2.5 \\
16.0 \\
16.5 \\
26.5\end{array}$ & $\begin{array}{l}540 \\
810 \\
680 \\
850 \\
660\end{array}$ & $\begin{array}{r}204 \\
\overline{-} \\
\overline{109}\end{array}$ \\
\hline SW22 & Orderville Gulch below $\$$ Sw19 & $\begin{array}{l}07-17-86 \\
09-09-86 \\
11-06-86 \\
09-18-87\end{array}$ & $\begin{array}{l}0.27 \\
0.13 \\
0.41 \\
0.07 \mathrm{E}\end{array}$ & $\begin{array}{r}23.0 \\
19.0 \\
0.5 \\
18.0\end{array}$ & $\begin{array}{l}780 \\
610 \\
800 \\
605\end{array}$ & $\begin{array}{r}1,840 \\
885 \\
- \\
-\end{array}$ \\
\hline SW23 & North fork of tributary to Orderville Gulch & $\begin{array}{l}07-17-86 \\
09-09-86 \\
11-06-86 \\
07-13-86\end{array}$ & $\begin{array}{l}0.23 \\
0.20 \\
0.32 \\
0.012\end{array}$ & $\begin{array}{r}17.0 \\
17.5 \\
6.0 \\
27.5\end{array}$ & $\begin{array}{r}610 \\
560 \\
580 \\
--\end{array}$ & $\begin{array}{r}581 \\
487 \\
-- \\
--\end{array}$ \\
\hline SWR4 & \multicolumn{6}{|c|}{ QLEAR CREEK DRAINAGE } \\
\hline \multirow[t]{2}{*}{$\begin{array}{l}\text { SW25 } \\
\text { SWR6 }\end{array}$} & $\begin{array}{l}\text { Clear Creek below Trough Hollow } \\
\text { Clear Creek }\end{array}$ & $\begin{array}{l}09-14-86 \\
09-14-86\end{array}$ & $\begin{array}{l}0.17 \\
0.14\end{array}$ & $\begin{array}{l}17.5 \\
13.0\end{array}$ & $\begin{array}{l}470 \\
430\end{array}$ & $\begin{array}{r}73 \\
3\end{array}$ \\
\hline & \multicolumn{6}{|c|}{ MEADOW CREEK DRAINAGE } \\
\hline $\begin{array}{l}\text { SWR7 } \\
\text { SW28 } \\
\text { SW29 }\end{array}$ & $\begin{array}{l}\text { Upper Little Meadow Creek } \\
\text { Meadow Creek below Burnt Flat } \\
\text { Meadow Creek near Highway } 9\end{array}$ & $\begin{array}{l}08-20-86 \\
08-19-86 \\
04-22-81 \\
08-26-81 \\
08-19-86\end{array}$ & $\begin{array}{l}0 .-- \\
0.021 \\
0.29 \\
0.09 \\
0.017\end{array}$ & $\begin{array}{l}18.0 \\
28.0 \\
13.0 \\
26.0 \\
25.0\end{array}$ & $\begin{array}{r}540 \\
2,100 \\
2,120 \\
2,340 \\
2,960\end{array}$ & $\begin{array}{r}\overline{46} \\
2,900 \\
5 \\
223\end{array}$ \\
\hline
\end{tabular}


Table 1.--Streamflow, temperature, specific conductance, and suspendedsediment concentration for selected streamflow sites-continued

\begin{tabular}{|c|c|c|c|c|c|c|}
\hline $\begin{array}{l}\text { Site } \\
\text { identifier }\end{array}$ & Stream & Date & $\begin{array}{l}\text { Stream- } \\
\text { flow, } \\
\text { instan- } \\
\text { taneous } \\
\left(\mathrm{ft}^{3} / \mathrm{s}\right)\end{array}$ & $\begin{array}{l}\text { Temper- } \\
\text { ature } \\
\left({ }^{\circ} \mathrm{C}\right)\end{array}$ & $\begin{array}{l}\text { Spe- } \\
\text { cific } \\
\text { con- } \\
\text { duct- } \\
\text { ance } \\
(\mu S / \mathrm{cm})\end{array}$ & $\begin{array}{l}\text { Sus- } \\
\text { pended- } \\
\text { sedi- } \\
\text { ment } \\
\text { concen- } \\
\text { tration } \\
\text { (mg/L) }\end{array}$ \\
\hline \multicolumn{7}{|c|}{ INSIDE ZION NARROWS } \\
\hline $\begin{array}{l}\text { ZSW1 } \\
\text { ZSWR }\end{array}$ & $\begin{array}{l}\text { North Fork Virgin River above Deep Creek } \\
\text { North Fork Virgin River at Deep Creek }\end{array}$ & $\begin{array}{l}09-16-86 \\
09-17-86 \\
09-18-86\end{array}$ & $\begin{array}{l}6.6 \\
6.7 \\
--\end{array}$ & $\begin{array}{c}16.0 \\
19.0\end{array}$ & $\begin{array}{r}515 \\
--\end{array}$ & $\overline{-}$ \\
\hline ZSWB & Deep Creek at mouth & $\begin{array}{l}09-17-86 \\
09-18-86\end{array}$ & $\begin{array}{l}9.1 \\
--\end{array}$ & ${ }^{1} 10.0$ & $41 \overline{0}$ & -- \\
\hline $\begin{array}{l}\text { ZSW4 } \\
\text { ZSW5 }\end{array}$ & $\begin{array}{l}\text { North Fork Virgin River below Kolob Creek } \\
\text { Goose Creek at mouth }\end{array}$ & $\begin{array}{l}06-09-87 \\
09-22-87 \\
09-18-86 \\
06-09-87 \\
09-22-87\end{array}$ & $\begin{array}{l}55 \\
28.0 \\
0.21 \\
0.3 \\
0.2\end{array}$ & $\begin{array}{l}18.0 \\
12.0 \\
13.5 \\
15.0 \\
15.0\end{array}$ & $\begin{array}{l}335 \\
305 \\
440 \\
375 \\
390\end{array}$ & $\begin{array}{l}- \\
- \\
-- \\
-\end{array}$ \\
\hline \multirow{3}{*}{$\begin{array}{l}\text { ZSW6 } \\
\text { ZSW7 } \\
\text { ZSW8 } \\
\text { ZSW9 }\end{array}$} & $\begin{array}{l}\text { North Fork Virgin River near Big Spring } \\
\text { North Fork Virgin River above Orderville Gulch }\end{array}$ & $\begin{array}{l}09-18-86 \\
06-10-87 \\
09-23-87\end{array}$ & $\begin{array}{l}27 \\
65.5 \\
40.9\end{array}$ & $\begin{array}{l}13.5 \\
16.5 \\
13.0\end{array}$ & $\begin{array}{l}540 \\
400 \\
450\end{array}$ & $\overline{-}$ \\
\hline & $\begin{array}{l}\text { Orderville Gulch at mouth } \\
\text { North Fork Virgin River at Temple of Sinawava }\end{array}$ & $\begin{array}{l}09-19-86 \\
08-19-81\end{array}$ & 1.5 & $\begin{array}{l}12.5 \\
20.5\end{array}$ & $\begin{array}{r}1,420 \\
580\end{array}$ & -- \\
\hline & & $\begin{array}{l}10-17-81 \\
02-11-82 \\
05-04-82 \\
09-19-86 \\
01-27-87\end{array}$ & $\begin{array}{r}54 \\
45 \\
700 \\
36 \\
--\end{array}$ & $\begin{array}{r}8.5 \\
6.0 \\
6.0 \\
13.0 \\
6.5\end{array}$ & $\begin{array}{l}540 \\
550 \\
325 \\
625 \\
580\end{array}$ & $\begin{array}{l}- \\
- \\
- \\
-\end{array}$ \\
\hline
\end{tabular}

- Measurements taken in early morning. All other temperature measurements at this station for September 16 to 19,1986 , were taken in late afternoon or early evening. 
The mean monthly streamflow at the two gaging stations in the study area were compared with the mean monthly precipitation at Alton for the periods of record indicated in figure 10. Although the Alton climatologic station is not in the Virgin River drainage basin, the Alton precipitation records were used because this climatologic station is closer in elevation to the elevations of the gaging stations than the climatologic station at $\mathrm{Zion}$, and precipitation at this station is assumed to be more representative of values for the lease tract. These comparisons show that melting of snow that fell during January through March provides the water for sustained high flows through April and May into June.

\section{Runoff and Evapotranspiration}

When mean monthly precipitation at Alton, in inches, is compared with runoff, in inches (fig. 11), as measured at SW2 on the North Fork Virgin River below Bulloch Canyon, the delay between snowpack accumulation and snowmelt runoff is even more apparent. If precipitation at Alton is assumed to be similar in type and quantity to that in the drainage area above SW2, an important condition is revealed during the period of thunderstorm runoff, July through september. During these months, though mean monthly precipitation values approach those of snow accumulation, less than half of the precipitation might become runoff.

Some of the precipitation may be recharging those formations that are permeable or fractured at the surface; however, most of the runoff probably is being lost by evapotranspiration. Annual potential evapotranspiration is estimated to be about 18 to 21 in./year in the northern part of the study area, increasing to 27 to $30 \mathrm{in} /$ year at lower elevations to the south (Jeppson and others, 1968, fig. 25). Plantz (1985, fig. 1) showed May through October pan evaporation in the study area to be 45 to 55 in. Both estimates at the lower elevations exceed the normal annual precipitation of 20 to 25 in.

\section{Peak and Average Annual Streamflow}

On the basis of streamflow records at SW2, SW4, and ZSW10, peak instantaneous streamflow usually is the result of summer thunderstorms in July, August, or September. From 1974 to 1984 , the maximum instantaneous streamflow recorded at SW2 was $272 \mathrm{ft}^{3} / \mathrm{s}$ on May 31, 1983; however, before the station was installed in 1974, a maximum streamflow of $1,740 \mathrm{ft}^{3} / \mathrm{s}$ on September 9, 1974, was determined on the basis of slope-area measurements (ReMillard and others, 1984, p. 233). During the period of record, about 45 percent of the annual peak instantaneous streamflow values at SW2 occurred from July through September and 36 percent occurred from March through May. Similarly, at ZSWl0, 44 percent of annual peak streamflow values from 1933 to 1974 are the result of summer (July, August, and September) thunderstorms (Maddox and others, 1977, p. 20).

Peak and average annual streamflows for ungaged drainages in the study area can be estimated using regression equations developed by Thamas and Lindskov (1983, tables 5, 6) and Christensen and others (1986, table 4). Selected equations are shown in table 2. If the mean basin elevation is greater than $8,000 \mathrm{ft}$ and the study site datum is greater than $7,000 \mathrm{ft}$, the 


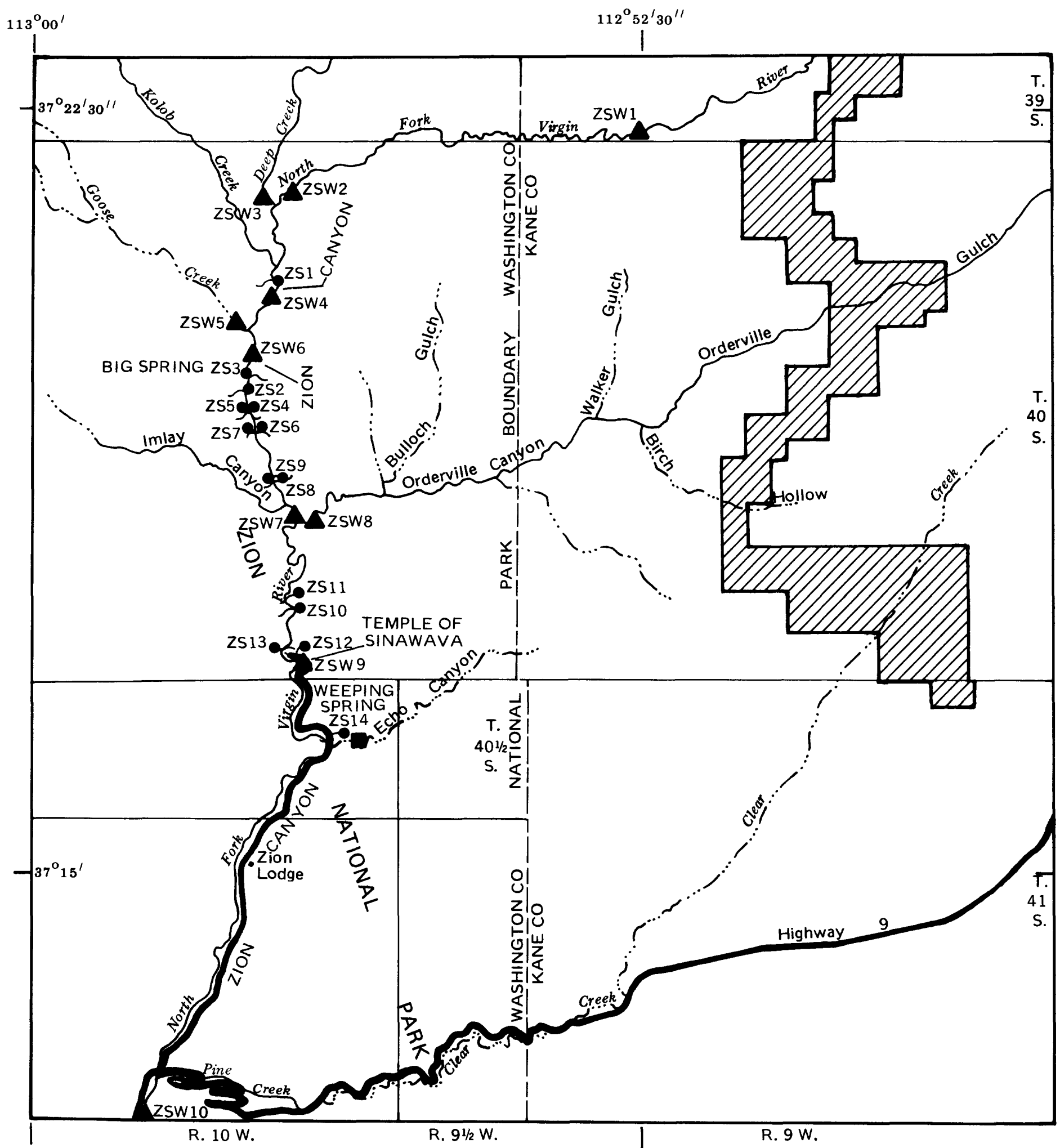

Base from U.S. Geological Survey $1: 100,000$

quadrangle: Kanab, Utah, 1980

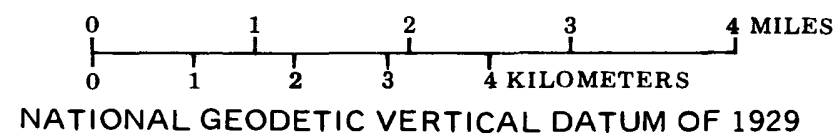

Figure 9.--Location of and selected water-quality diagrams for springs, streamflow sites, and a stable isotope sample site in Zion Canyon. 

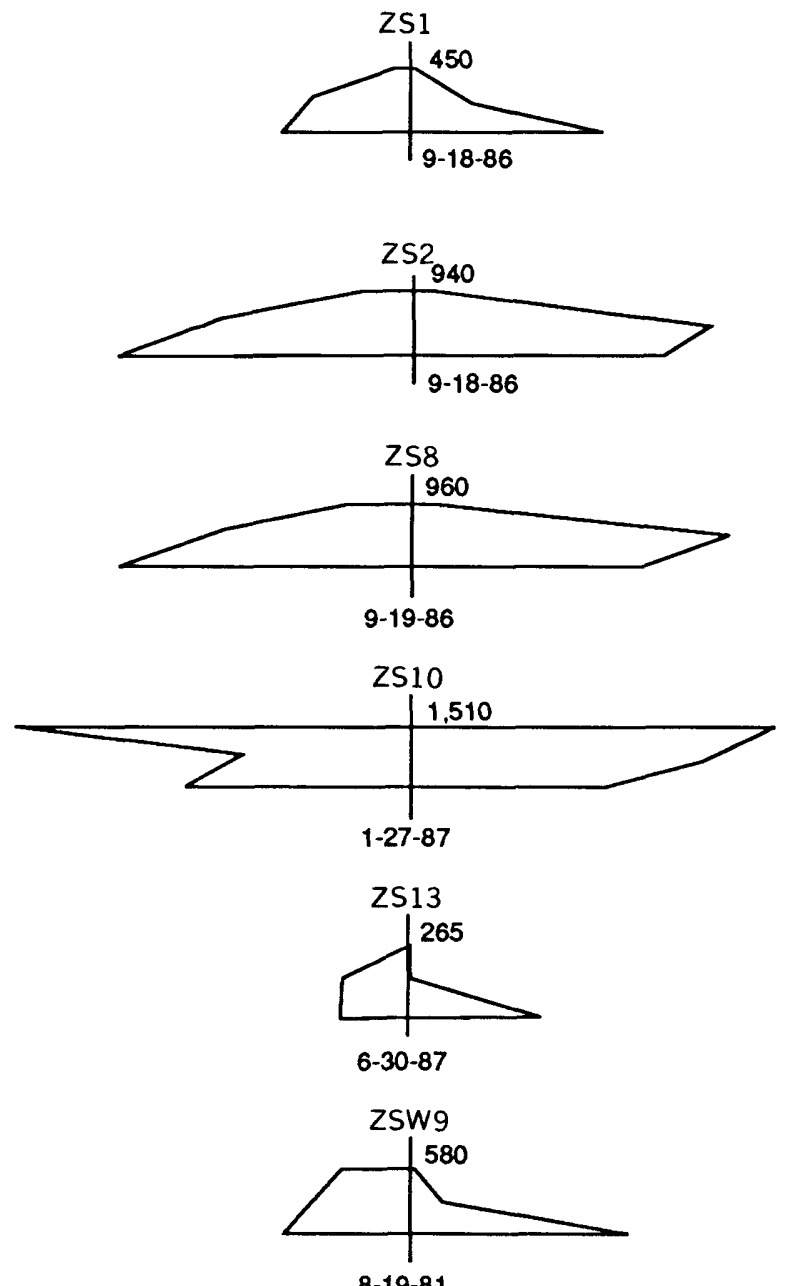

8-19-81

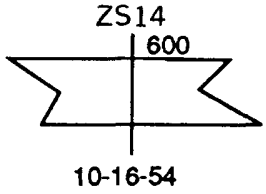

EXPLANATION

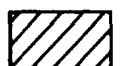

L.C. HOLDING COAL-LEASE TRACT

ZS14 ZSW9

SPRING AND IDENTIFIER

STREAMFLOW SITE AND IDENTIFIER

- STABLE ISOTOPE SAMPLE SITE

WATER-QUAUTY DIAGRAM

(Modified from Stiff, 1951)

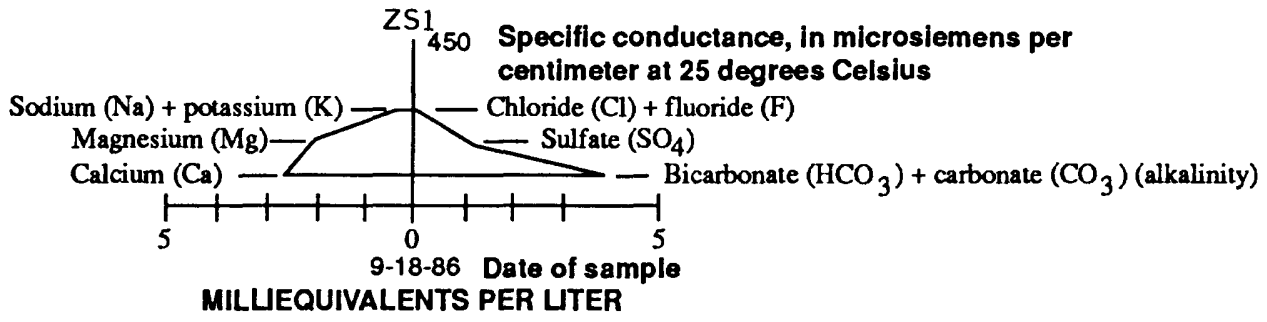

Figure 9.--Location of and selected water-quality diagrams for springs, streamflow sites, and a stable isotope sample site in Zion Canyon--Continued. 

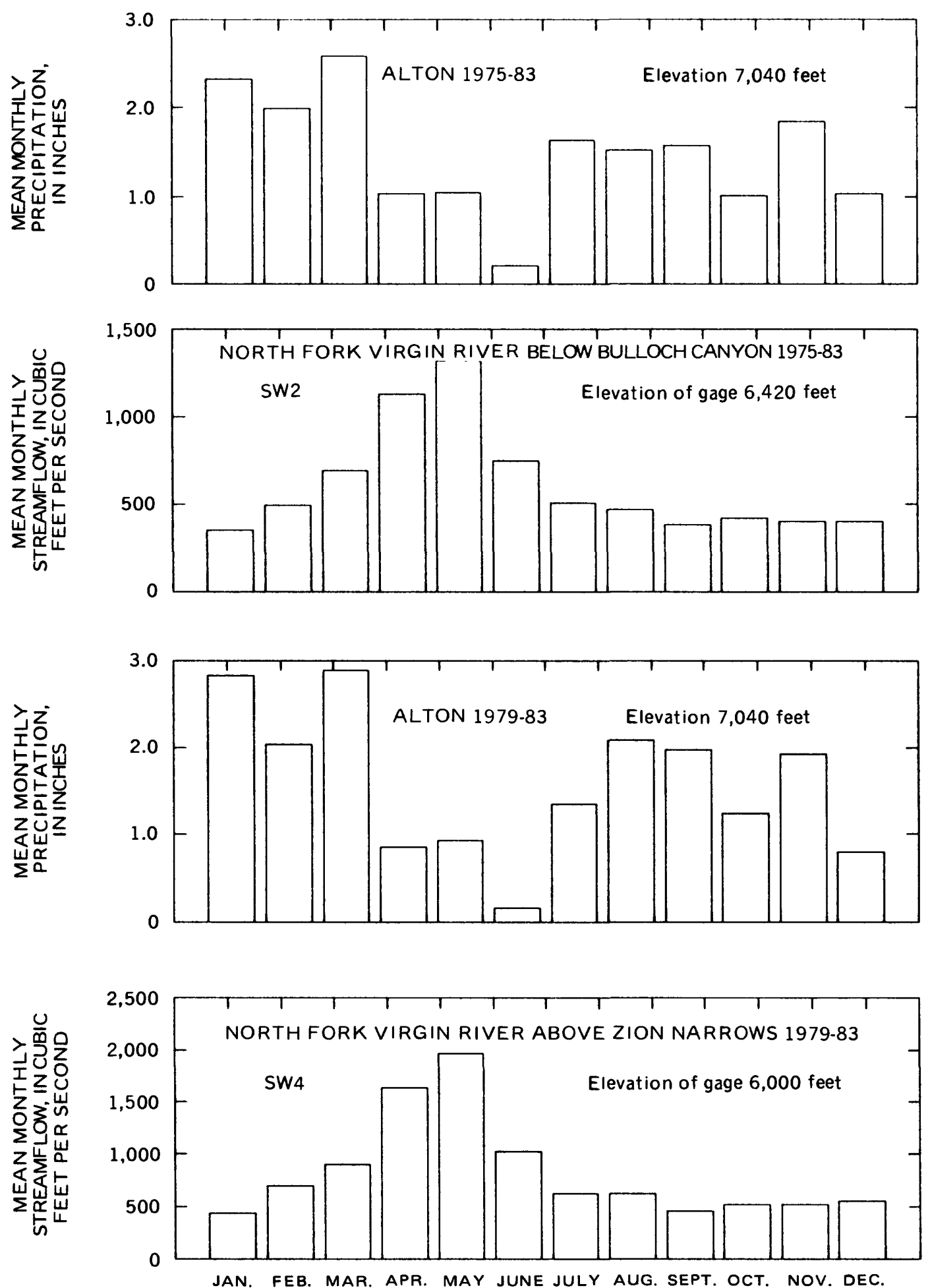

Figure 10.--Mean monthly precipitation at Alton and mean monthly streamflow at two gaging stations on the North Fork Virgin River. 


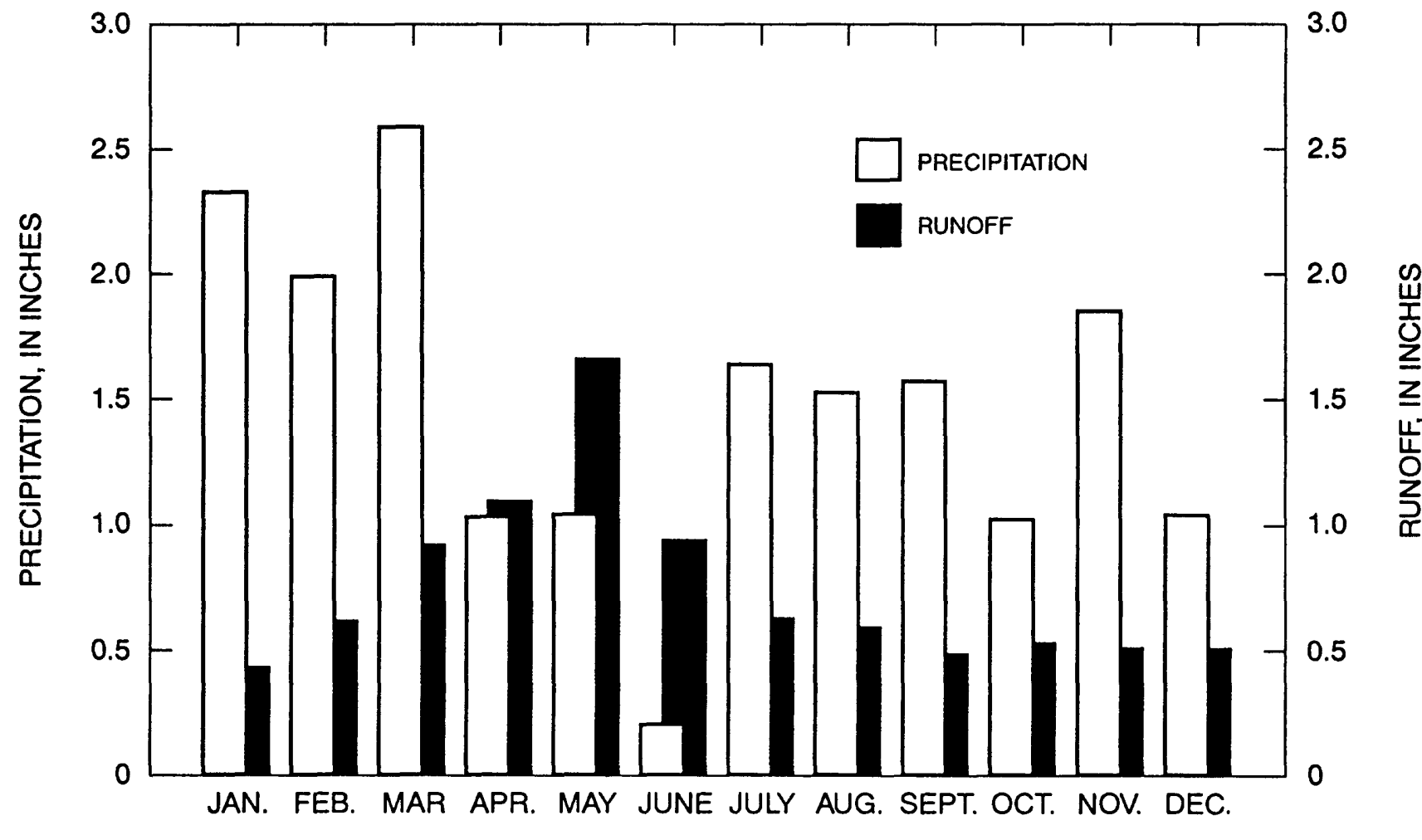

Figure 11.--Mean monthly precipitation at Alton and mean manthly runoff at North Fork Virgin River below Bulloch Canyon,SW2, 1975-83.

equations of set $A$ are used. The equations in set $B$ are used when the mean basin elevation is less than $8,000 \mathrm{ft}$ or the site datum is less than 7,000 $\mathrm{ft}$.

In order to calculate the peak and average annual streamflows at a particular site with these equations, the contributing drainage basin area and the mean basin elevation were determined. The contributing drainage basin area in square miles, $A$, was determined by digitizing or planimetering the drainage area from a topographic map. The mean basin elevation in thousands of feet, E, was determined by placing a transparent grid over the topographic map of the drainage area. The elevations of a minimum of 20 equally spaced points were tabulated, and the average elevation of the points was calculated.

The peak streamflows with 10- and 100-year recurrence intervals and the average streamflow at SW21 for the creek in Orderville Gulch were computed to demonstrate the use of the equations in table 2. The contributing drainage basin area above SW21 is $14.9 \mathrm{mi}^{2}$, and the mean basin elevation is 7.31 thousand $\mathrm{ft}$; therefore, the equations for a mean basin elevation less than $8,000 \mathrm{ft}$ (set B) were used. The estimated 10-year peak streamflow at SW21 is $904 \mathrm{ft}^{3} / \mathrm{s}$, and the 100 -year peak is $2,900 \mathrm{ft}^{3} / \mathrm{s}$. The average annual streamflow at this site is estimated to be $7.92 \mathrm{ft}^{3} / \mathrm{s}$. 
Table 2.--Regression equations used to compute peak and average annual streamflows from ungaged tributaries in the L.C. Holding coal-lease tract area

Equation: Q, streamflow, in cubic feet per second; A, drainage area, in square miles; E, mean basin elevation, in thousands of feet

- SET A -

FOR AREAS WITH MEAN BASIN ELLVVATION GREATER THAN 8,000 FEET AND A STUDY SITE DATUM GREATER THAN 7,000 FEET

Recurrence

interval,

in years

Equation

Peak Streamflow

(Thamas and Lindskov, 1983, table 5)

10

$Q=680\left(A^{0.706}\right)\left(E^{-1.30}\right)$

50

$Q=64,200\left(A^{0.651}\right)\left(E^{-3.03}\right)$

100

$Q=347,000\left(A^{0.631}\right)\left(E^{-3.68}\right)$

Average Annual Streamflow

(Christensen and others, 1986, table 4)

$\mathrm{Q}=3.54+0.294 \mathrm{~A}$

-- SET B -

FOR AREAS WITH MEAN BASIN ELEVATION LESS THAN 8,000 FEET OR A STUDY SITE DATUM LESS THAN 7,000 FEET

Recur rence

interval,

in years

Equation

Peak Streamflow

(Thomas and Lindskov, 1983, table 6)

$10 \quad Q=23,700\left(A^{0.433}\right)\left(E^{-2.23}\right)$

$50 \quad Q=61,000\left(A^{0.375}\right)\left(E^{-2.19}\right)$

100

$Q=83,100\left(A^{0.356}\right)\left(E^{-2.17}\right)$

Average Annual Streamflow

(Christensen and others, 1986, table 4)

- $\quad Q=3.54+0.294 \mathrm{~A}$ 
Thomas and Lindskov (1983, table 12) compared station flood-frequency values (peak streamflow determined from station records) with weighted floodfrequency values (average of station flood-frequency and peak streamflow computed from the regression equations such as those in table 2) for the North Fork Virgin River near Springdale (ZSW10). The difference between the station flood-frequency values and the weighted values for the 2-, 5-, 10-, 25-, 50-, and 100-year floods was 6 percent or less, suggesting that the regression equations provide reasonable estimates for the study area. Similarly, estimates of weighted average annual streamflow by Christensen and others (1986, table 6) for sites ZSW10 and SW2 were within 3 percent of the values determined from station records.

\section{Water Quality}

Surface-water samples were collected from several streams in the study area. The results of analyses for chemical quality are shown in table 3 and suspended-sediment concentrations are shown in table 4. Data from previous studies are also included in tables 3 and 4.

\section{Chemical Quality}

Diagrams showing chemical composition of surface water in the L.C. Holding coal-lease tract (pl. 2) indicated that the chemical composition of the North Fork Virgin River was about the same from upstream to downstream sample sites despite the addition of water with larger dissolved-solids concentrations from some tributaries. In addition, the composition did not appreciably change during the 15-month sampling period. Calcium, magnesium, and bicarbonate plus carbonate are the major ions in the river. Dissolvedsolids concentrations (sum of constituents) range from 190 to $280 \mathrm{mg} / \mathrm{L}$ for the sites shown on plate 2 (table 3 ).

Streamflow entering the North Fork Virgin River from tributaries such as the stream in Hay Canyon (SWI3) and an unnamed tributary (SW16), has larger dissolved-solids concentrations, from 640 to $1,000 \mathrm{mg} / \mathrm{L}$, and magnesium, calcium, sulfate, and bicarbonate plus carbonate are the major constituents (pl. 2). Inflow from these tributaries is small compared to the total streamflow of the North Fork Virgin River, thus, the chemical composition of the river remains relatively unchanged by their contributions.

Streamflow in the upstream reaches of the North Fork Virgin River has relatively small dissolved-solids concentrations because the flow is largely derived from rainfall and snowmelt that have been in contact with rock and soil for a relatively short time (Sandberg and Sultz, 1985, p. 10). Small dissolved-solids concentrations; the prominence of calcium, magnesium, and bicarbonate plus carbonate; and the similarity in composition to spring water in the Wasatch Formation (Cordova, 1981, fig. 13, p. 44) confirm that base flow to the North Fork Virgin River is largely from springs and seeps (Sandberg and Sultz, 1985, p. 6). Larger dissolved-solids concentrations and larger magnesium and sulfate concentrations in some tributary waters indicate that their base-flow sources are waters that have longer travel times in formations and may come in contact with magnesium- and sulfate-bearing strata. For example, the chemical composition of streamflow at SW13, the stream in Hay 
Table 3.-Streamflow and chemical quality of $\left[\mathrm{ft}^{3} / \mathrm{s}\right.$, cubic feet per second; ${ }^{\circ} \mathrm{C}$, degrees Celsius;

Site identifier: Locations shown on plate 2 or figure 9.

Streamf low: Current meter or flume measurement except E, estimated.

Alkal inity: Field values except $L$, laboratory value.

$\mathrm{mg} / \mathrm{L}$, milligrams per liter:

\begin{tabular}{|c|c|c|c|c|c|c|c|c|}
\hline $\begin{array}{l}\text { Site } \\
\text { identi- } \\
\text { fier }\end{array}$ & Stream & Date & $\begin{array}{l}\text { Stream- } \\
\text { flow } \\
\left(\mathrm{ft}^{3} / \mathrm{s}\right)\end{array}$ & $\begin{array}{l}\text { Temper- } \\
\text { ature } \\
\left({ }^{\circ} \mathrm{C}\right)\end{array}$ & $\begin{array}{l}\text { Spe- } \\
\text { cific } \\
\text { con- } \\
\text { duct- } \\
\text { ance } \\
(\mu \delta / \mathrm{cm})\end{array}$ & $\begin{array}{c}\text { pH } \\
\text { (stand- } \\
\text { and } \\
\text { units) }\end{array}$ & $\begin{array}{l}\text { Calcium, } \\
\text { dis- } \\
\text { solved } \\
\text { (mg/L } \\
\text { as Ca) }\end{array}$ & $\begin{array}{l}\text { Magne- } \\
\text { sium, } \\
\text { dis- } \\
\text { solved } \\
\text { (mg/L } \\
\text { as } \mathrm{Mg})\end{array}$ \\
\hline
\end{tabular}

\section{VIRGIN RIVER DRAINAGE}

SWI North Fork Virgin River below Rosy Canyon

SWR North Fork Virgin River below Bulloch Canyon

SWB North Fork Virgin River near Willow Canyon

SH4 North Fork Virgin River above Zion Narrows

SW5 North Fork Virgin River above Bulloch's cabin

SW6 North Fork Virgin River below Bulloch's cabin

ZSW9 North Fork Virgin River at Temple of Sinawava

SW13 Hay Canyon below Millet Canyon

SW16 Unnamed tributary to North Fork Virgin River

$06-11-86$
$07-16-86$
$06-12-86$
$06-18-86$
$04-22-81$

13

13

19

17

11.0

14.0

16.5

20.0

08-20-81

08-26-81

$10-08-81$

$05-03-82$

08-23-82

06-18-86

$07-16-86$

07-29-87

09-18-87

7.5

11.0

7.5
6.9
7.3
34
13

16.0

16.5

14.5

14.5

22.0

$18 \quad 14.5$

14.5
20.0

-- -

08-19-81

10-17-81

02-11-82

05-04-82

44
54
45
700

06-13-86

07-16-86

09-12-86

$\begin{array}{llr}0.06 & 28.5 & 1,390 \\ 0.14 & 24.0 & 1,360 \\ 0.07 & 17.5 & 980\end{array}$

345
390
360
385
520
420
415
435
455
360
430
410
395
425

580
540
550
325
1,390
1,360
980

\section{4}

8.4

8.5

8.6

8.6
8.5

$\begin{array}{ll}52 & 17 \\ 55 & 18 \\ 54 & 20 \\ 47 & 20 \\ 59 & 28\end{array}$

\section{4}

8.4

8.5

8.0

8.4

54

50

\section{5}

8.6

8.5

8.3

55

48

41

7.0

8.6

8.3

8.5

$$
54
$$

54
59

60
46

$\begin{array}{rrr}8.4 & 110 & 110 \\ 8.4 & 140 & 110 \\ 8.4 & 79 & 61\end{array}$

\section{ORDERVILLF GLLCH DRAINAGE}

Sw19 Orderville Eulch below Twins Hollow

SWRO Orderville Eulch above roadcrossing

SWR1 Orderville Eulch at roadcrossing

SW22 Orderville Gulch below SW19 SW25 Clear Creek below Trough Hollow
SW26 Clear Creek

SW29 Meadow Creek near Highway 9

$06-22-86$
$06-22-86$
$06-21-86$
$09-18-87$

$\begin{array}{ll}0.04 & 22.5 \\ 0.25 & 26.5 \\ 0.24 & 26.5\end{array}$

$0.07 \mathrm{E} \quad 18.0$

\section{CLEAR CREEK DRAINAGE}

$\begin{array}{lllllll}09-14-86 & 0.17 & 17.5 & 470 & 8.2 & 69 & 21 \\ 09-14-86 & 0.14 & 13.0 & 430 & 8.4 & 54 & 22\end{array}$

MEADOW CREEK DRAINAGE
08-19-86
0.01725 .0
2,960
8.5
97
150

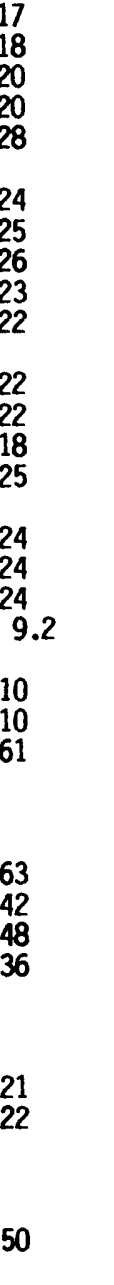


water from selected streamflow sites

$\mu \mathrm{S} / \mathrm{cm}$, microsiemens per centimeter at 25 degrees Celsius;

$\mu \mathrm{g} / \mathrm{L}$, micrograms per liter]

\begin{tabular}{|c|c|c|c|c|c|c|c|c|c|c|c|}
\hline $\begin{array}{l}\text { Sodium, } \\
\text { dis- } \\
\text { solved } \\
\text { (mg/L } \\
\text { as } \mathrm{Na} \text { ) }\end{array}$ & $\begin{array}{l}\text { Potas- } \\
\text { sium, } \\
\text { dis- } \\
\text { solved } \\
(\mathrm{mg} / \mathrm{L} \\
\text { as K) }\end{array}$ & $\begin{array}{l}\text { Alka- } \\
\text { linity, } \\
\text { total } \\
\text { field } \\
(\mathrm{mg} / \mathrm{L} \text { as } \\
\left.\mathrm{CaCO}_{3}\right)\end{array}$ & $\begin{array}{c}\text { Sulfate, } \\
\text { dis- } \\
\text { solved } \\
\text { (mg/L } \\
\text { as } \mathrm{SO}_{4} \text { ) }\end{array}$ & $\begin{array}{l}\text { Chlo- } \\
\text { ride, } \\
\text { dis- } \\
\text { solved } \\
\text { (mg/L } \\
\text { as Cl) }\end{array}$ & $\begin{array}{l}\text { Fluo- } \\
\text { ride, } \\
\text { dis- } \\
\text { solved } \\
\text { (mg/L } \\
\text { as F) }\end{array}$ & $\begin{array}{l}\text { Silica, } \\
\text { dis- } \\
\text { solved } \\
(\mathrm{mg} / \mathrm{h} \\
\text { as } \\
\left.\mathrm{SiO}_{2}\right)\end{array}$ & $\begin{array}{l}\text { Solids, } \\
\text { residue } \\
\text { at } 180 \\
{ }^{\circ} \mathrm{C}, \\
\text { dis- } \\
\text { solved } \\
\text { (mg/L) }\end{array}$ & $\begin{array}{l}\text { Solids, } \\
\text { sum of } \\
\text { consti- } \\
\text { tuents, } \\
\text { dis- } \\
\text { solved } \\
\text { (mg/L) }\end{array}$ & $\begin{array}{l}\text { Hard- } \\
\text { ness } \\
(\mathrm{mg} / \mathrm{L} \\
\text { as } \\
\left.\mathrm{CaCO}_{3}\right)\end{array}$ & $\begin{array}{l}\text { Manga- } \\
\text { nese, } \\
\text { dis- } \\
\text { solved } \\
(\mu \mathrm{g} / \mathrm{L} \\
\text { as } \mathrm{Mn})\end{array}$ & $\begin{array}{l}\text { Iron, } \\
\text { dis- } \\
\text { solved } \\
\text { (ug/ } / \\
\text { as Fe) }\end{array}$ \\
\hline \multicolumn{12}{|c|}{ VIRGIN RIVER DRAINAGE } \\
\hline $\begin{array}{l}1.4 \\
1.7 \\
2.6 \\
3.8 \\
7.5\end{array}$ & $\begin{array}{l}1.0 \\
1.2 \\
1.1 \\
1.2 \\
1.4\end{array}$ & $\begin{array}{l}205 \\
219 \\
209 \\
194 \\
140 \mathrm{~L}\end{array}$ & $\begin{array}{l}9.5 \\
8.3 \\
20 \\
23 \\
59\end{array}$ & $\begin{array}{l}1.1 \\
1.2 \\
1.6 \\
1.8 \\
2.3\end{array}$ & $\begin{array}{l}0.2 \\
0.2 \\
0.2 \\
0.2 \\
0.3\end{array}$ & $\begin{array}{l}9.9 \\
11 \\
11 \\
10 \\
9.1\end{array}$ & $\begin{array}{c}\overline{214} \\
\overline{--} \\
300\end{array}$ & $\begin{array}{l}220 \\
230 \\
240 \\
220 \\
250\end{array}$ & $\begin{array}{l}200 \\
210 \\
220 \\
200 \\
260\end{array}$ & $\begin{array}{l}3 \\
3 \\
8 \\
3 \\
6\end{array}$ & $\begin{array}{r}3 \\
9 \\
17 \\
13 \\
40\end{array}$ \\
\hline $\begin{array}{l}8.1 \\
7.7 \\
8.6 \\
4.8 \\
5.4\end{array}$ & $\begin{array}{l}1.5 \\
1.6 \\
1.4 \\
1.2 \\
1.5\end{array}$ & $\begin{array}{l}180 \mathrm{~L} \\
180 \mathrm{~L} \\
210 \mathrm{~L} \\
190 \mathrm{~L} \\
177 \mathrm{~L}\end{array}$ & $\begin{array}{l}39 \\
40 \\
44 \\
39 \\
27\end{array}$ & $\begin{array}{l}2.1 \\
8.0 \\
3.5 \\
2.3 \\
2.0\end{array}$ & $\begin{array}{l}0.1 \\
0.1 \\
0.2 \\
0.2 \\
0.2\end{array}$ & $\begin{array}{l}12 \\
13 \\
12 \\
8.5 \\
12\end{array}$ & $\begin{array}{l}234 \\
-- \\
-- \\
--\end{array}$ & $\begin{array}{l}250 \\
250 \\
280 \\
250 \\
220\end{array}$ & $\begin{array}{l}230 \\
230 \\
240 \\
230 \\
200\end{array}$ & $\begin{array}{l}-- \\
4 \\
-- \\
-- \\
--\end{array}$ & $\begin{array}{c}-- \\
<10 \\
-- \\
--\end{array}$ \\
\hline $\begin{array}{l}5.7 \\
5.4 \\
4.9 \\
8.6\end{array}$ & $\begin{array}{l}1.2 \\
1.5 \\
1.5 \\
1.7\end{array}$ & $\begin{array}{l}212 \\
218 \\
153 L \\
164 L\end{array}$ & $\begin{array}{l}34 \\
29 \\
23 \\
42\end{array}$ & $\begin{array}{l}1.8 \\
1.9 \\
1.5 \\
1.5\end{array}$ & $\begin{array}{l}0.2 \\
0.2 \\
0.2 \\
0.2\end{array}$ & $\begin{array}{l}11 \\
11 \\
10 \\
12\end{array}$ & $\begin{array}{l}-- \\
201 \\
206 \\
243\end{array}$ & $\begin{array}{l}260 \\
250 \\
190 \\
--\end{array}$ & $\begin{array}{l}230 \\
210 \\
180 \\
--\end{array}$ & $\begin{array}{l}3 \\
3 \\
6 \\
2\end{array}$ & $\begin{array}{r}16 \\
46 \\
28 \\
4\end{array}$ \\
\hline $\begin{array}{l}30 \\
27 \\
27 \\
4.5\end{array}$ & $\begin{array}{l}2.8 \\
2.7 \\
2.7 \\
1.4\end{array}$ & $\begin{array}{l}150 \mathrm{~L} \\
160 \mathrm{~L} \\
170 \mathrm{~L} \\
130 \mathrm{~L}\end{array}$ & $\begin{array}{l}85 \\
83 \\
83 \\
11\end{array}$ & $\begin{array}{c}35 \\
33 \\
31 \\
6.4\end{array}$ & $\begin{array}{l}0.1 \\
0.2 \\
0.2 \\
0.1\end{array}$ & $\begin{array}{c}11 \\
11 \\
9.9 \\
6.7\end{array}$ & $\begin{array}{l}-- \\
-- \\
--\end{array}$ & $\begin{array}{l}330 \\
340 \\
340 \\
160\end{array}$ & $\begin{array}{l}230 \\
250 \\
250 \\
150\end{array}$ & $\begin{array}{l}-- \\
-- \\
--\end{array}$ & $\begin{array}{l}-- \\
-- \\
--\end{array}$ \\
\hline $\begin{array}{l}24 \\
20 \\
29\end{array}$ & $\begin{array}{l}5.6 \\
9.2 \\
4.9\end{array}$ & $\begin{array}{l}258 \\
306 \\
194\end{array}$ & $\begin{array}{l}510 \\
550 \\
330\end{array}$ & $\begin{array}{l}10 \\
17 \\
12\end{array}$ & $\begin{array}{l}0.4 \\
0.4 \\
0.2\end{array}$ & $\begin{array}{l}9.4 \\
9.7 \\
9.4\end{array}$ & $\begin{array}{r}1,080 \\
680\end{array}$ & $\begin{array}{r}930 \\
1,000 \\
640\end{array}$ & $\begin{array}{l}730 \\
800 \\
450\end{array}$ & $\begin{array}{l}7 \\
24 \\
12\end{array}$ & $\begin{array}{r}22 \\
19 \\
9\end{array}$ \\
\hline \multicolumn{12}{|c|}{ ORDERVILLE GULCH DRAINAGE } \\
\hline $\begin{array}{l}15 \\
12 \\
26 \\
19\end{array}$ & $\begin{array}{l}3.7 \\
3.0 \\
3.4 \\
2.7\end{array}$ & $\begin{array}{l}411 \\
210 \\
192 \\
165 L\end{array}$ & $\begin{array}{l}220 \\
150 \\
200 \\
130\end{array}$ & $\begin{array}{l}6.1 \\
4.9 \\
6.2 \\
3.1\end{array}$ & $\begin{array}{l}0.3 \\
0.2 \\
0.2 \\
0.2\end{array}$ & $\begin{array}{l}10 \\
8.8 \\
9.1 \\
7.5\end{array}$ & $\begin{array}{l}-- \\
-- \\
--\end{array}$ & $\begin{array}{l}670 \\
410 \\
470 \\
350\end{array}$ & $\begin{array}{l}530 \\
330 \\
340 \\
270\end{array}$ & $\begin{array}{r}65 \\
4 \\
3 \\
4\end{array}$ & $\begin{array}{r}38 \\
7 \\
9 \\
8\end{array}$ \\
\hline \multicolumn{12}{|c|}{ CLEAR CREEK DRAINAGE } \\
\hline $\begin{array}{l}2.6 \\
2.8\end{array}$ & $\begin{array}{l}1.9 \\
1.8\end{array}$ & $\begin{array}{l}238 \\
232\end{array}$ & $\begin{array}{l}23 \\
25\end{array}$ & $\begin{array}{l}2.6 \\
2.7\end{array}$ & $\begin{array}{l}0.1 \\
0.1\end{array}$ & $\begin{array}{l}7.5 \\
7.6\end{array}$ & $\begin{array}{l}263 \\
259\end{array}$ & $\begin{array}{l}269 \\
259\end{array}$ & $\begin{array}{l}260 \\
230\end{array}$ & $\begin{array}{l}7 \\
6\end{array}$ & $\begin{array}{l}16 \\
14\end{array}$ \\
\hline \multicolumn{12}{|c|}{ MEADOW CREEK DRAINAGE } \\
\hline 410 & 15 & 326 & 1,400 & 36 & 0.3 & 4.1 & 2,400 & 2,300 & 860 & 20 & 20 \\
\hline
\end{tabular}




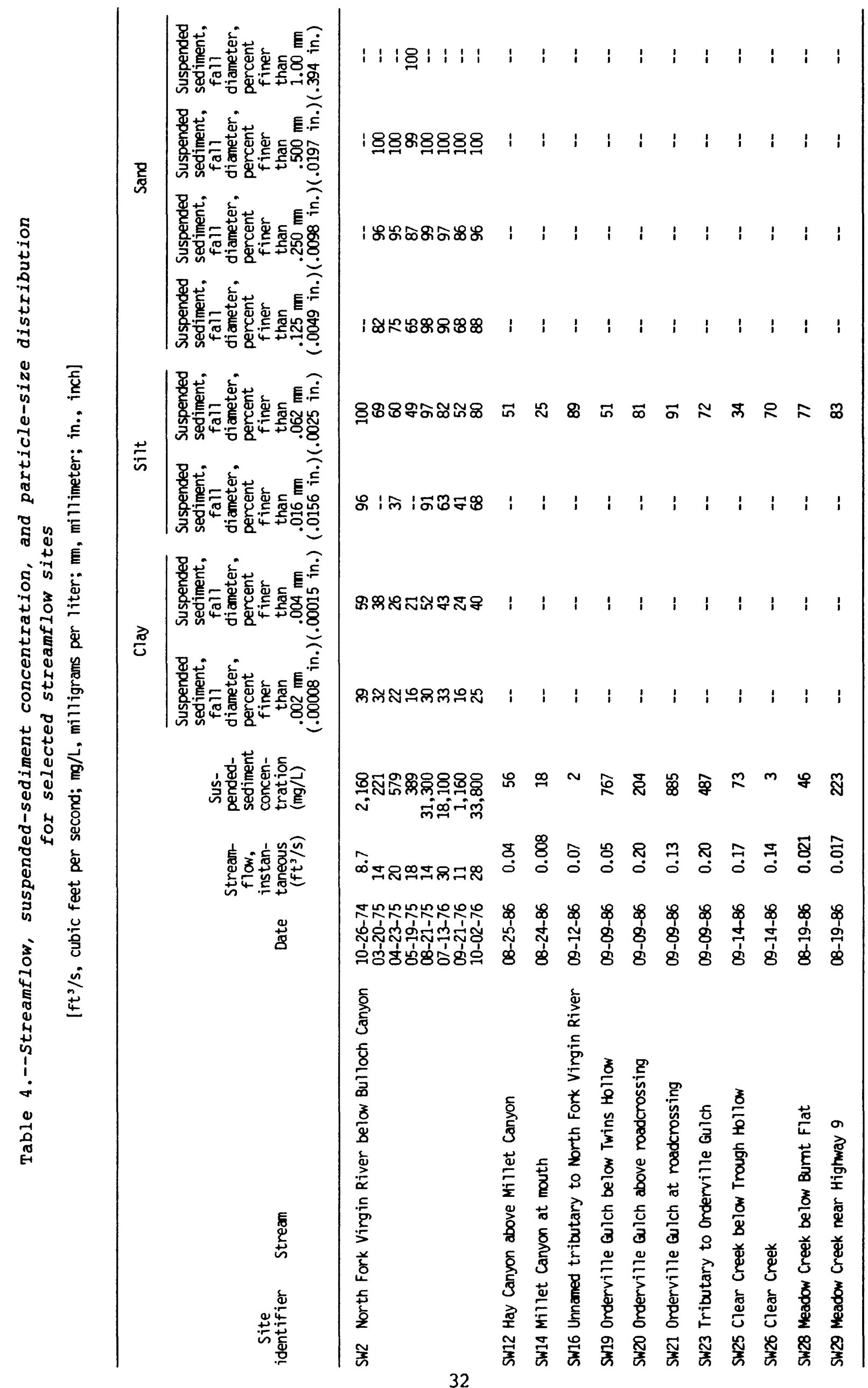


Canyon (table 8), is very similar to water from springs S4 and S5 (see "Ground-Water Hydrology" section of this report) in the drainage upstream from SN13.

Specific-conductance values, which are a measure of the ability of a specific volume of a solution to conduct electrical current at a specific temperature, are also indicative of dissolved-solids concentrations. A linear relation exists between specific conductance and dissolved-solids concentration under most conditions such that dissolved solids, in mg/L, is generally 0.55 to 0.75 of specific conductance, in $\mu \mathrm{S} / \mathrm{am}$ at $25^{\circ} \mathrm{C}$ (Hem, 1985 , p. 67).

A comparison of specific-conductance values measured in 1986 during low streamflow (November) and moderately high streamflow (June) for the North Fork Virgin River (table 1) indicates that specific conductance generally is larger during periods of low streamflow. Conductance decreases during periods of moderate to high streamflow as a result of dilution by rain or snowmelt runoff.

Dissolved-solids concentrations of 4 water samples from Orderville Gulch range from 350 to $670 \mathrm{mg} / \mathrm{L}$ (table 3 ), and specific-conductance values in 14 samples range from 540 to $990 \mu \mathrm{S} / \mathrm{cm}$ (table 1). Predominant ions are magnesium, calcium, bicarbonate, and sulfate. On June 22, 1986, the dissolved-solids concentration decreased between sites SW19 and SW20 (table $3)$, probably because of the seepage and tributary inflow of water with smaller dissolved-solids concentration.

On the basis of two samples collected on September 14, 1986, water quality in Clear Creek is similar to that of the North Fork Virgin River; calcium, magnesium, and bicarbonate were the predominant ions. Dissolvedsolids concentrations were 260 and $230 \mathrm{mg} / \mathrm{L}$, and specific-conductance values were 470 and $430 \mu \mathrm{S} / \mathrm{Cm}$ at sites SW25 and SW26, respectively.

A water sample ollected August 19, 1986, from Meadow Creek (site SW29) had a dissolved-solids concentration of $2,300 \mathrm{mg} / \mathrm{L}$ and specific conductance of $2,960 \mu \mathrm{S} / \mathrm{cm}$ (table 3 ). The predominant constituents were sodium and sulfate. At the time of sampling, the streamflow was relatively warm, $25^{\circ} \mathrm{C}$, and the discharge was very small, $0.017 \mathrm{ft}^{3} / \mathrm{s}$ (table 3). The major ions in water from a spring in the Meadow Creek drainage (S22, pl. 1) were sodium, calcium, sulfate, and bicarbonate. Evaporation of spring water of this composition could cause calcite to precipitate resulting in water of similar composition to that of Meadow Creek.

Discharge, temperature, and specific conductance were measured at eight streamflow sites (ZSW2-ZSW9) in Zion Canyon and one site upstream from the canyon (ZSWI)(fig. 9). Data are shown in table 1. Complete chemical analyses of streamflow at ZSW9 are shown in table 3.

Specific-conductance values measured during September 1986 for the North Fork Virgin River generally increased downstream through $\mathrm{Z}$ ion Canyon from $480 \mu \mathrm{S} / \mathrm{cm}$ at ZSW2 to $625 \mu \mathrm{S} / \mathrm{cm}$ at the Temple of Sinawava (ZSW9)(table 1). The specific-conductance values for surface water from Deep and Goose Creeks, averaging about $425 \mu \mathrm{s} / \mathrm{cm}$, were slightly less than that of flow in the North Fork Virgin River. In contrast, the stream in Orderville Gulch had a specific 
conductance of $1,420 \mu \mathrm{S} / \mathrm{cm}$ (ZSW8), but it accounted for a very minor part of the streamflow into zion Canyon. The increase in conductance values downstream can be attributed primarily to inflow of ground-water with large dissolved-solids concentrations from the east side of zion Canyon as discussed in the section of this report on ground-water quality.

\section{Suspended Sediment}

Streamflow, suspended-sediment concentration, and particle-size distribution were determined for 11 streamflow samples collected during this study from several drainages in the study area (table 4). The same types of data are also presented for site SW2 from October 1974 through October 1976 (table 4). Suspended-sediment concentrations for samples without associated particle-size distributions are shown in table 1.

Data collected during this study showed suspended-sediment concentrations ranging from $2 \mathrm{mg} / \mathrm{L}$ (SW16) to $1,840 \mathrm{mg} / \mathrm{L}$ (SW21) (table 1). Previous data for SW2 on the North Fork Virgin River included concentrations ranging from 221 $\mathrm{mg} / \mathrm{L}$ to $33,800 \mathrm{mg} / \mathrm{L}$ (table 4 ).

Based on limited data from site SW2, the largest suspended-sediment concentrations are associated with runoff from intense thunderstorms in the summer and fall (fig. 12). Although the streamflow resulting from thunderstorm runoff may be less than streamflow associated with snowmelt runoff, the sediment concentrations are several times larger. For example, the streamflow on April 23, 1975, was $20 \mathrm{ft}^{3} / \mathrm{s}$ with a suspended-sediment concentration of $579 \mathrm{mg} / \mathrm{L}$. There was no precipitation at Alton or Zion on April 23 or for several preceding days (National Oceanic and Atmospheric Administration, 1975a) and thus, the streamflow is considered snowmelt runoff. In contrast, streamflow of $14 \mathrm{ft}^{3} / \mathrm{s}$ on August 21, 1975, carried a suspendedsediment concentration of $31,300 \mathrm{mg} / \mathrm{L}$. Precipitation records (National Oceanic and Atmospheric Administration, 1975b) showed that 0.61 and 0.11 in. of precipitation fell at Alton and Zion, respectively, on that date, and the streamflow is considered to be thunderstorm runoff.

The large suspended-sediment concentrations associated with storm runoff can be explained by considering the intense erosional forces of falling rain. In the study area, thunderstorms are characterized by large quantities of rain falling in a short time over a relatively small area. The raindrops loosen soil and rock particles and carry them in sheet wash or rills downslope into tributary channels that quickly drain into the major streams.

The principal author of this report witnessed the runoff in the North Fork Virgin River during a summer thunderstorm. The flow in this river is normally clear enough to see the river bottom (fig. 13A). On July 20, 1987, following several hours of rain in the upper reaches of the North Fork Virgin River, the discharge in the river downstream from SW4 had increased, causing overbank flooding. The bottam of the river was not visible through the brown, sediment-laden water (fig. 13B). 


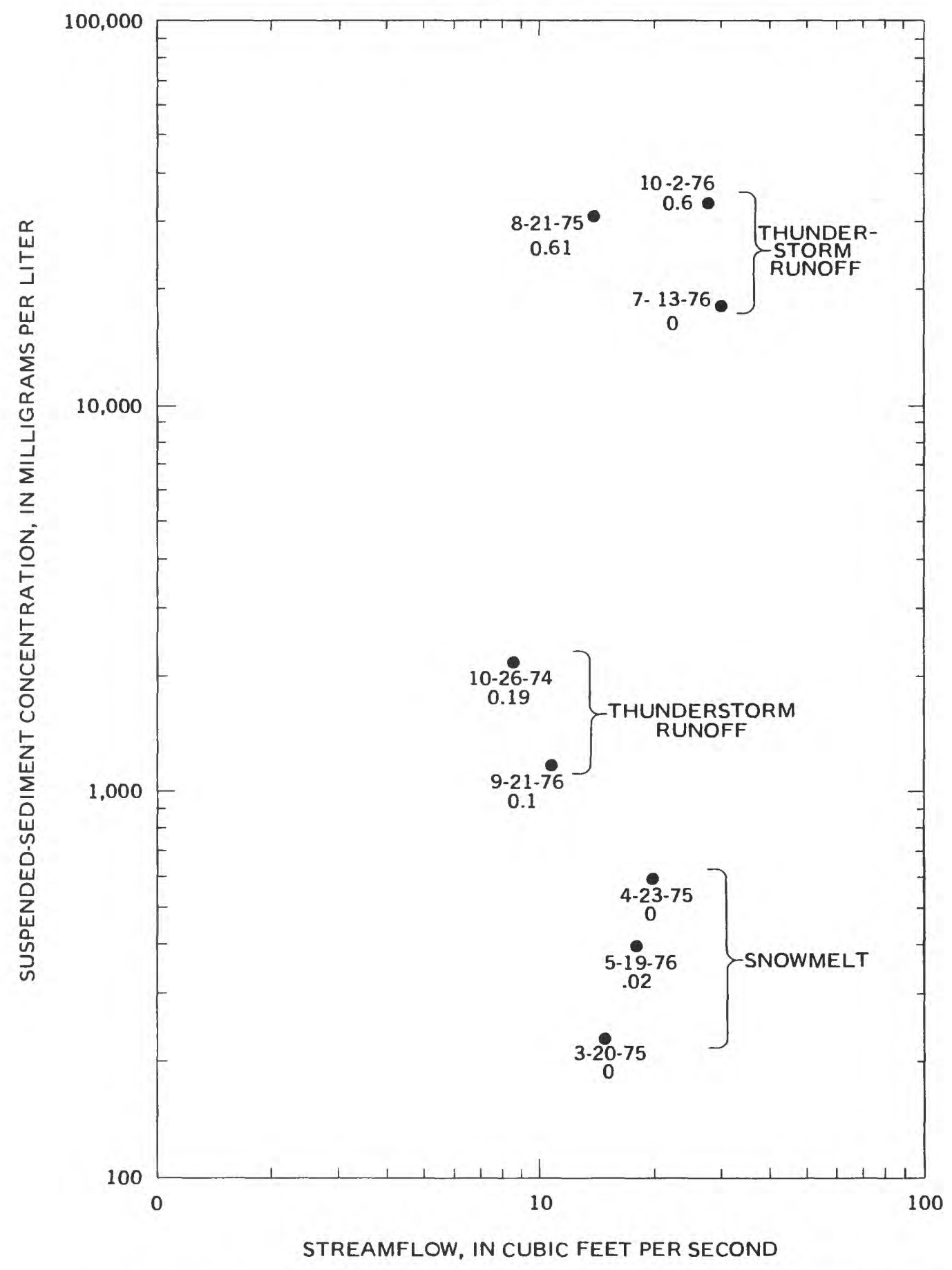

Figure 12.--Relation of streamflow to suspended-sediment concentration at site SW2, North Fork Virgin River below Bulloch Canyon, October 1974 through October 1976. Date of sample and precipitation at Alton, in inches, are shown. 


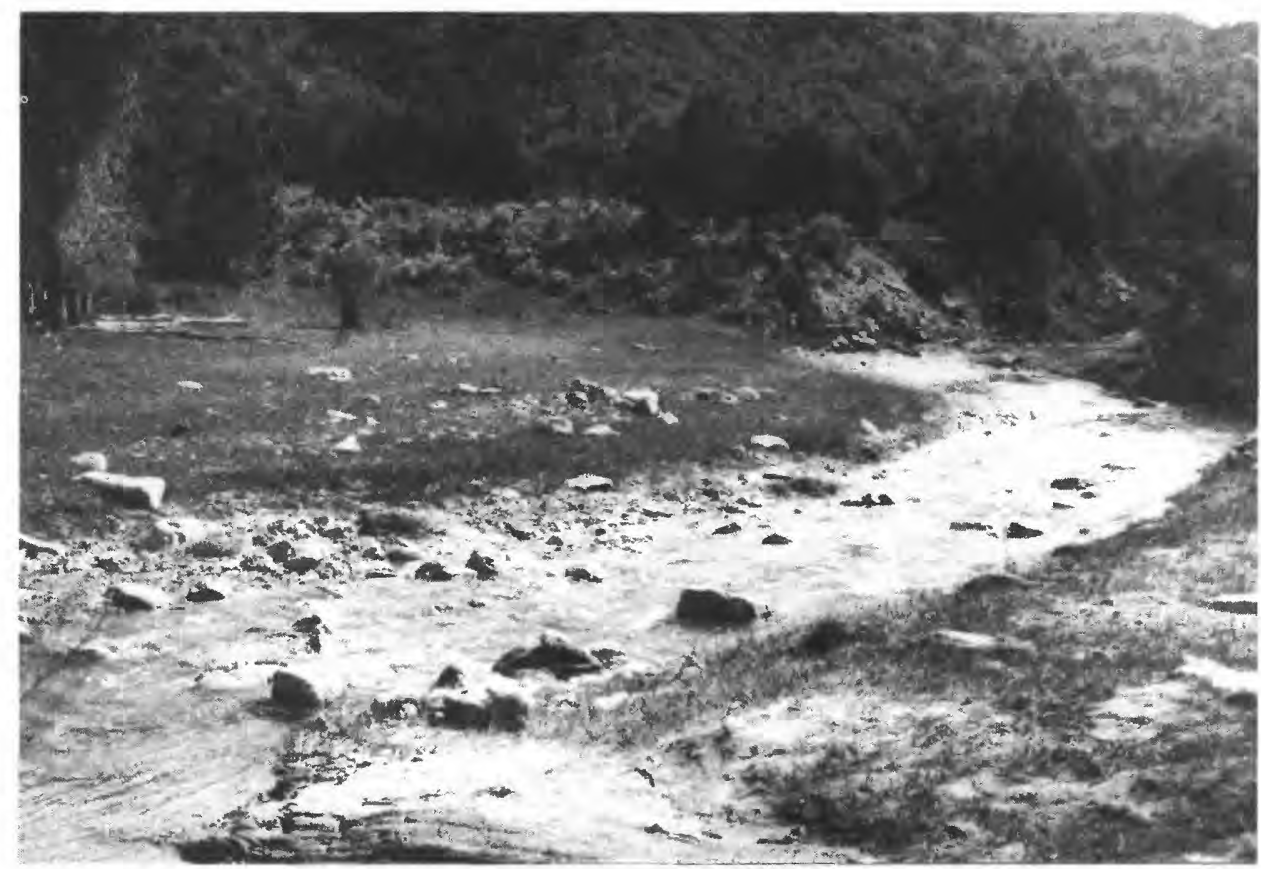

A--Low flow is clear; rocky stream bottom visible.

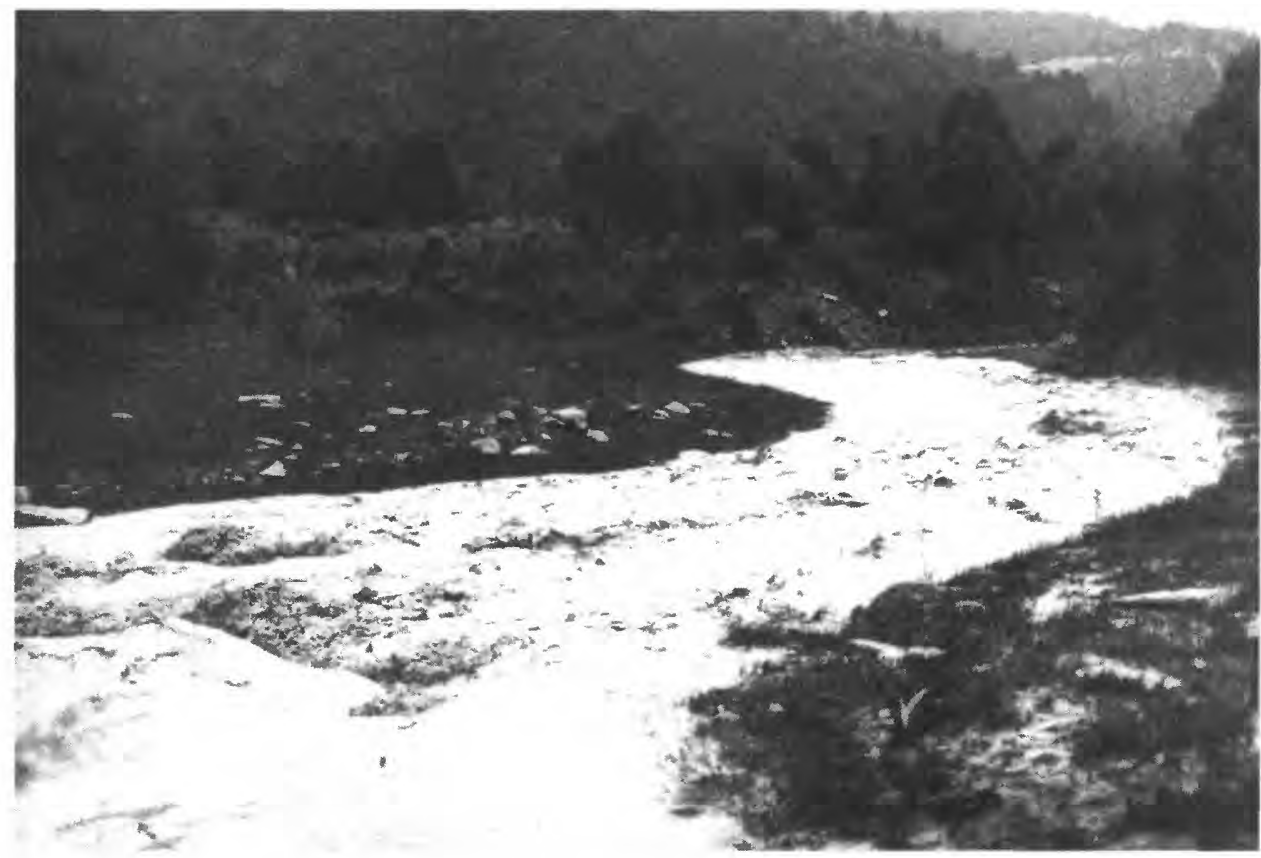

B--Storm runoff is sediment-laden.

Figure 13.--Sediment load in the North Fork Virgin River before (A) and during (B) thunderstorm runoff. (Photograph taken about one mile downstream from SW4.) 
The percentage of sand to silt and clay in suspended sediment was determined for 11 samples collected during this investigation. The data in table 4 indicate that more than 50 percent of the sediment in nine of the samples consisted of silt- and clay-size particles. In seven of the samples, 70 to 91 percent of the sediment consisted of silt and clay. Particle-size distributions for eight samples taken at site SW2 on the North Fork Virgin River (table 4), indicate that even during high flows the suspended sediment is predominantly silt and clay.

The abundance of silt and clay in the suspended-sediment reflects the textural character of rocks in the study area. Particles forming fine-grained sandstone, siltstone, and shale are common in formations such as the Tropic Shale and Carmel Formation. Erosion and sediment yield are discussed in the following section.

\section{Erosion and Sediment Yield}

The volume of material that is removed from the surface of the land by erosion is defined as sediment yield. Sediment yield is directly related to the rate of erosion. Factors that influence the erosion rate include soil type, climate, extent and type of vegetation, and topographic factors such as stream gradient and slope.

In the lease tract, land use in the form of grazing livestock has also affected the rate of erosion. Grazing in this area began in the 1800's and possibly before that time, and by the turn of the century, most of the rangelands were deteriorating, and the most palatable plants were scarce (Turner, 1949). The result was accelerated erosion in the form of headward cutting, gullying, and drainage of naturally irrigated meadowlands (Turner, 1949 , p. 11). The lease tract now is used as summer range for small numbers of sheep and cattle.

A large-scale map of sediment yield by Price (1987, p. 39), based largely on the geology of the area, shows that, with the exception of the landslide areas in the southern part of the study area (pl. 2), annual sediment yields are generally in the moderate range of 0.2 to $0.5 \mathrm{acre}-\mathrm{ft} / \mathrm{mi}^{2}$. The annual sediment yield in areas of landslide activity is in the range of 0.5 to 1.0 acre-ft/mi $\mathrm{mi}^{2}$ (Price, 1987, p. 39).

Estimates of annual sediment yield can be made on a smaller scale using the PSIAC method (Pacific Southwest Inter-Agency Comittee, 1968). Using this method, the various factors that affect erosion and sediment yield such as geology, climate, and ground cover are given numerical ratings (table 5). The sum of these ratings is representative of a sediment-yield class with a given range of yield as follows (Pacific Southwest Inter-Agency Committee, 1968, p. $(-2)$ : 
Estimated average annual sediment yield,

Rating

Class in acre- $\mathrm{ft} / \mathrm{mi}^{2}$

$\begin{array}{lll}\text { Greater than } 100 & 1 & \text { Greater than } 3.0 \\ 75-100 & 2 & 1.0-3.0 \\ 50-75 & 3 & 0.5-1.0 \\ 25-50 & 4 & 0.2-0.5 \\ 0-25 & 5 & \text { Less than } 0.2\end{array}$

Thus, a summary rating of 60 for the lease tract (table 5) indicates an annual sediment yield in the range of 0.5 to $1.0 \mathrm{acre}-\mathrm{ft} / \mathrm{mi}^{2}$.

From the PSIAC rating ranges and sediment-yield values, Shown (1970, p. B246) prepared a graph, plotting the upper values of the rating ranges and those of the corresponding estimated sediment yields, from which a specific value for the yield estimate could be determined. By this method, the estimated annual source-area sediment yield for the coal-lease tract is about 0.7 acre-ft/mi ${ }^{2}$.

Data on suspended-sediment loads, in tons, for gaging stations at SW2 and SW4 are shown in table 6. These data reflect the wide variation possible in annual sediment load. If the density of the sediment is assumed to be 1,525 tons/acre-ft (value taken from an area draining similar types of formations) (Utah Division of Water Resources, 1979, p. 41), then the annual suspendedsediment yields are $0.08 \mathrm{acre}-\mathrm{ft} / \mathrm{mi}^{2}$ at SW2 and $0.12 \mathrm{acre}-\mathrm{ft} / \mathrm{mi}^{2}$ at SW4. These yields are smaller than the values estimated by the PSIAC method because the yields from gaging stations only reflect the suspended-sediment, whereas the PSIAC estimates include all material, both in the bedload and in suspension, that is removed from the land surface. In addition, use of a density value determined specifically for the study area could result in different values of suspended-sediment yields.

\section{GROUND-WATER HYDROLOGY}

\section{Occurrence of Ground Water}

In order to obtain data on ground-water occurrence, water levels, and water quality, particularly for the formations underlying the coal, holes ranging from 158 to $985 \mathrm{ft}$ were drilled at four locations in the northwestern part of the study area along the North Fork Virgin River. Piezameters were installed in each drillhole to monitor water levels. Three of the drillholes contain dual completions--two piezometers monitoring saturated zones at different depths. Locations of all drillholes are shown on plate 2 . Pertinent data for the monitoring wells are given in table 7, and lithologic logs are shown in the "Supplemental Information" section at the end of this report. 
Table 5.--Rating factors for estimating source-area sediment yield for the L.C. Holding coal-lease tract

(Pacific Southwest Inter-Agency Committee, 1968)

\begin{tabular}{|c|c|c|c|c|c|}
\hline \multirow[t]{2}{*}{ Parameter } & \multirow[t]{2}{*}{ Description } & \multirow[t]{2}{*}{ Rating } & \multicolumn{3}{|c|}{ Range } \\
\hline & & & Low & Moderate & High \\
\hline Surface geology & $\begin{array}{l}\text { Predominantly easily eroded shale and weakly } \\
\text { to moderately cemented sandstone; some interbedded } \\
\text { conglomerate, siltstone, and limestone. }\end{array}$ & 7 & 0 & 5 & 10 \\
\hline Soils & $\begin{array}{l}\text { Mainly rock outcrop; little or no soil development } \\
\text { particularly on shale. Percolation and infiltration } \\
\text { minimal in shale and siltstone. High shrink-swell } \\
\text { potential and easy dispersal of fine-textured soils } \\
\text { overlying Tropic Shale. }\end{array}$ & 6 & 0 & 5 & 10 \\
\hline Climate & $\begin{array}{l}\text { Based on precipitation at Alton, } 40 \text { to } 50 \text { percent } \\
\text { falls as snow; about } 30 \text { percent falls as rain during } \\
\text { short, intense thunderstorms. }\end{array}$ & 7 & 0 & 5 & 10 \\
\hline Runoff & $\begin{array}{l}\text { Large runoff during spring snowmelt; peak flaws } \\
\text { from thunderstorms. }\end{array}$ & 5 & 0 & 5 & 10 \\
\hline Topography & $\begin{array}{l}\text { Plateau-like upland areas cut by steep-sided canyons; } \\
\text { high relief; little flood-plain development. }\end{array}$ & 15 & 0 & 10 & 20 \\
\hline Ground cover & $\begin{array}{l}\text { Mostly trees and shrubs with little understory; good } \\
\text { grass cover in meadows. }\end{array}$ & 0 & -10 & 0 & 10 \\
\hline Land use & $\begin{array}{l}\text { Livestock grazing in meadows; hikers in lower canyons. } \\
\text { One main road used frequently during summer. }\end{array}$ & -10 & -10 & 0 & 10 \\
\hline
\end{tabular}


Table 5.--Rating factors for estimating source-area sediment yield for the L.C. Holding coal-lease tract-Continued

Parameter Description

Rating

Range

Low Moderate High

$\begin{array}{llll}\text { Upland erosion } & \text { Landslides and gully erosion common in Tropic } & 20 & 0 \\ & \text { Shale; gully and rill erosion cormon in weakly } & 10 \\ & \text { cemented sandstone. } & 10 & 0 \\ \text { Channel erosion } & \text { Ephemeral streams in soft bedrock; narrow channels } & 10 \\ \text { and sediment } & \text { with steep gradients. Occasional erosion of alluvial } & 25 \\ \text { transportation } & \text { stream banks; lange flows of medium (snowmelt runoff) } & & \\ & \text { and short (thunderstorm) duration; moderate flow depths. }\end{array}$


Table 6.--Estimates ${ }^{1}$ of suspended-sediment load for gaging stations along the North Fork Virgin River below Bulloch Camyon (SW2) and above Zion Narrows (SW4)

$\left[\mathrm{mi}^{2}\right.$, square miles; $\mathrm{ft}^{3} / \mathrm{sec}-$ days, cubic feet per second-days; acre-ft, acre-foot]

Site SW2--North Fork Virgin River below Bulloch Canyon (09405420)

Drainage area: $35.7 \mathrm{mi}^{2}$ for $1975-77$; revised to $29.6 \mathrm{mi}^{2}$ in 1978

\begin{tabular}{lcc}
\hline Water year & $\begin{array}{c}\text { Annual } \\
\text { suspended-sediment } \\
\text { load (tons) }\end{array}$ & $\begin{array}{c}\text { Annual } \\
\text { water discharge } \\
\text { (ft }{ }^{3} / \text { sec-days) }^{-}\end{array}$ \\
\hline 1975 & 2,755 & 4,593 \\
1976 & 2,454 & 3,716 \\
1977 & 2,842 & 2,349 \\
1978 & 8,427 & 6,690 \\
TOTAL & 16,478 & 17,348 \\
MEAN & 4,120 & 4,337
\end{tabular}

Average annual suspended-sediment load: 128 tons $/ \mathrm{mi}^{2}-0.08 \mathrm{acre}-\mathrm{ft} / \mathrm{mi}^{2}$

Site SW4-North Fork Virgin River above Zion Narrows (09405450)

Drainage area: $45.5 \mathrm{mi}^{2}$

\begin{tabular}{lcr}
\hline Water year & $\begin{array}{c}\text { Annual } \\
\text { suspended-sediment } \\
\text { load (tons) }\end{array}$ & $\begin{array}{c}\text { Annual } \\
\text { water discharge } \\
\text { (ft } \text { (sec-days) }^{3} \text { sec-das }\end{array}$ \\
\hline 1979 & 16,076 & 10,553 \\
1980 & 5,790 & 14,011 \\
1981 & 959 & 5,709 \\
1982 & 2,231 & 5,536 \\
1983 & 23,463 & 12,779 \\
1984 & 2,440 & 7,531 \\
TOTAL & 50,959 & 56,119 \\
MEAN & 8,493 & 9,353
\end{tabular}

Average annual suspended-sediment load: 186.7 tons $/ \mathrm{mi}^{2}-0.12 \mathrm{acre}-\mathrm{ft} / \mathrm{mi}^{2}$

1 Estimates of annual suspended-sediment load are from U.S. Geological Survey Water-Data Reports for Utah, water years 1975-84, except the 1979 value, which was recalculated due to an error in the original published value. 
Site identifier: Locations shown on plate 2 and figure 14.

Omer or user: Refers to last known owner or user.

Use of water: $H$, domestic or household; $S$, stock; U, unused.

Casing: Diameter: Reported from driller's log or measured onsite; Finish: 0, open end; P, perforated; S, Dashes (--) indicate no data available.

Geologic unit: Zone of perforation; Ktc, coal-bearing menber of Tropic Shale; Carmel Formation: Jcw, Winsor Elevation of land surface: Above National Geodetic Vertical Datum of 1929.

Yield: gal/min, gallons per minute.

Other data available: C, chemical analysis (table 8); L, lithologic log in "Supplemental Information" at end

\begin{tabular}{|c|c|c|c|c|c|c|c|c|}
\hline \multirow{2}{*}{$\begin{array}{l}\text { Site } \\
\text { ident- } \\
\text { ifier }\end{array}$} & \multirow[b]{2}{*}{ Location } & \multirow[b]{2}{*}{ Omer or user } & \multirow[b]{2}{*}{$\begin{array}{c}\text { Year } \\
\text { drilled }\end{array}$} & \multirow{2}{*}{$\begin{array}{l}\text { Use } \\
\text { of } \\
\text { water }\end{array}$} & \multirow{2}{*}{$\begin{array}{l}\text { Depth } \\
\text { of well } \\
\text { (feet) }\end{array}$} & \multicolumn{3}{|c|}{ Casing } \\
\hline & & & & & & $\begin{array}{l}\text { Diameter } \\
\text { (inches) }\end{array}$ & $\begin{array}{l}\text { Depth } \\
\text { (feet) }\end{array}$ & Finish \\
\hline \multirow[t]{2}{*}{ LCI } & (C-39-9)27daa-1 & U.S.G.S. & 1987 & u & 985 & 2 & 580 & P 548-580 \\
\hline & 27 daa-2 & do. & do. & do. & do. & 1 & 66 & P 41-62 \\
\hline \multirow[t]{2}{*}{ LQ2 } & $32 \mathrm{dcb}-1$ & do. & do. & do. & 359 & 2 & 356 & S $344-354$ \\
\hline & $32 \mathrm{dcb}-2$ & do. & do. & do. & do. & 1 & 147 & $00-172$ \\
\hline \multirow[t]{2}{*}{ LC3 } & 33 cba-1 & do. & do. & do. & 158 & 2 & 157 & P 124-157 \\
\hline & $33 c b a-2$ & do. & to. & do. & do. & 1 & 5 & $0 \quad 0-115$ \\
\hline LCA & $35 b c c-1$ & do. & do. & do. & 420 & 2 & 385 & P 324-344 \\
\hline $\begin{array}{l}\text { W1 } \\
\text { W2 } \\
\text { W3 }\end{array}$ & $\begin{array}{r}(C-41-9) 10 c d d-1 \\
13 b b c-1 \\
14 c a b-1\end{array}$ & $\begin{array}{l}\text { H. Drews } \\
\text { R. Hall } \\
\text { C. Wright }\end{array}$ & $\begin{array}{l}1966 \\
1970 \\
1966\end{array}$ & $\begin{array}{l}H \\
U \\
H\end{array}$ & $\begin{array}{l}186 \\
350 \\
380\end{array}$ & $\begin{array}{l}6 \\
6 \\
6\end{array}$ & $\begin{array}{r}186 \\
350 \\
47\end{array}$ & $\begin{array}{l}\text { P. } 150-180 \\
\text { P. } 280-348 \\
0\end{array}$ \\
\hline W4 & $15 \mathrm{aad}-1$ & H. Drews & 1966 & H & 147 & 6 & 147 & P $120-145$ \\
\hline $\begin{array}{l}\text { W5 } \\
\text { W6 }\end{array}$ & $\begin{array}{l}15 c d a-1 \\
15 d c d-1\end{array}$ & $\begin{array}{l}\text { Thurston } \\
\text { H. Drews }\end{array}$ & $\overline{1966}$ & $\begin{array}{l}u \\
\text { S }\end{array}$ & $\overline{245}$ & $-\overline{6}$ & $\overline{119}$ & $\overline{0}$ \\
\hline w7 & $15 d d b-1$ & T. Baca & 1961 & $H$ & 231 & 6 & 73 & 0 \\
\hline W8 & $20 b d b-1$ & $\begin{array}{l}\text { U.S. National } \\
\text { Park Service }\end{array}$ & 1962 & H & 925 & 6 & 925 & P 881-921 \\
\hline
\end{tabular}


selected wells

screened. Upper and lower limits of perforations or screen given in feet below the land surface, if known. Member; Jcb, banded member; Jcl, limestone member; Jt, Temple Cap Sandstone; JTrn, Navajo Sandstone. of report.

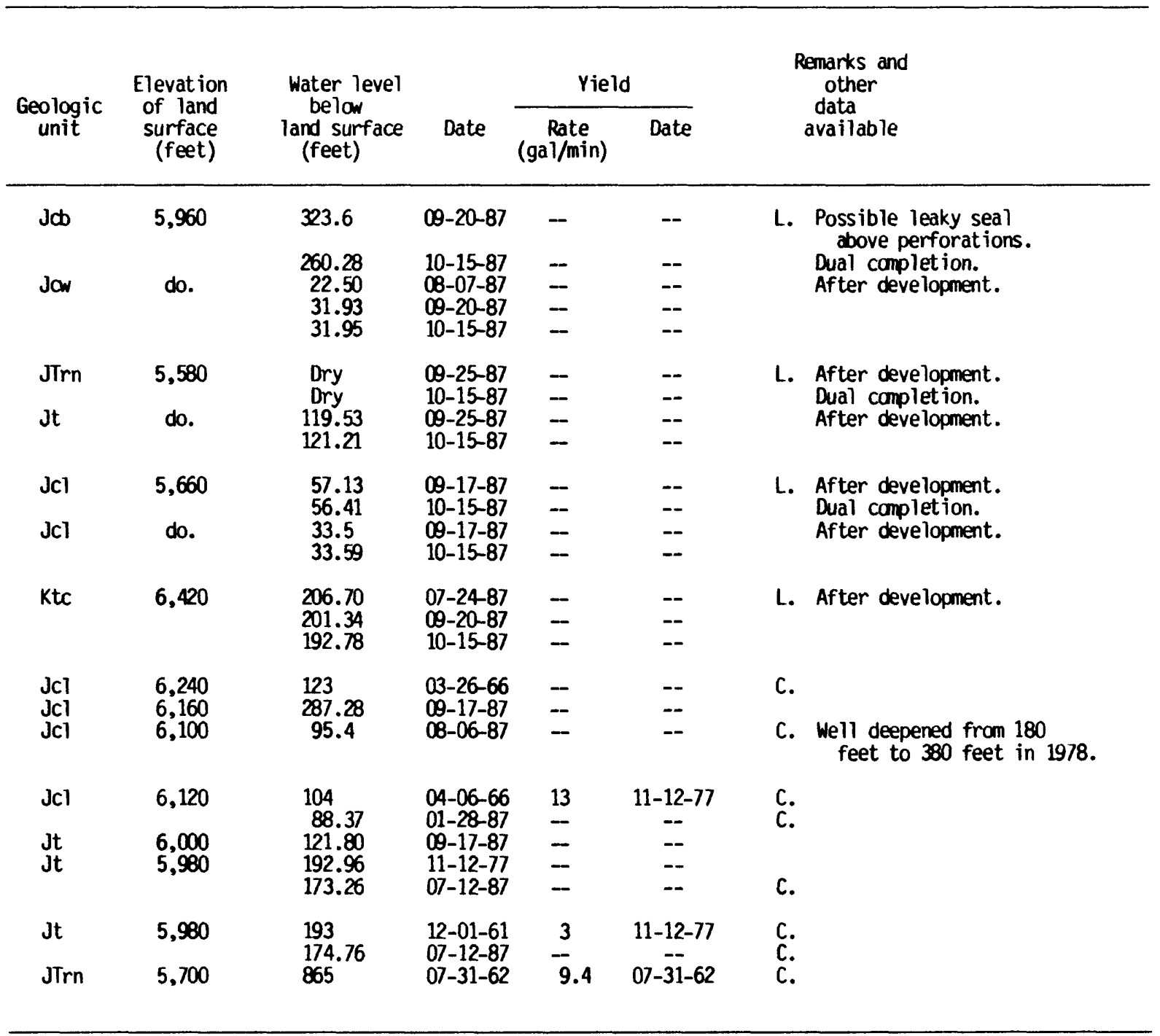


Ground water in the study area occurs in both unconsolidated and consolidated rocks. Saturated zones have been documented in alluvium (gal, pl. 1) along the North Fork Virgin River and its tributaries, and in parts of nearly all formations from the Wasatch Formation to the Navajo Sandstone.

The thickness of the alluvium varies considerably over short distances but thicknesses are generally 30 to $100 \mathrm{ft}$ (Cordova, 1981, p. 19). During this investigation, a shallow drillhole (later abandoned) in T. 39 S., R. 9 W. , sec. 34, SW $\frac{1}{4} S W^{\frac{1}{4}} \mathrm{NW} \frac{1}{4}$, penetrated about $42 \mathrm{ft}$ of alluvium along the North Fork Virgin River. No ground water was noted to a depth of $15 \mathrm{ft}$. At this point, the use of drilling fluid precluded the determination of the first show of ground water and the measurement of the water level. Unless the volume of ground water entering the drillhole is substantially larger than the volume of drilling fluid being used, the first occurrence or depth to ground water in the hole cannot be determined during drilling. Shallow holes drilled in 1979 for a dam-site investigation along the North Fork Virgin River near T. 39 S.,

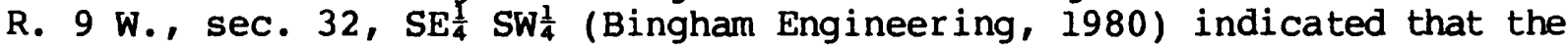
thickness of alluvium ranged from $23 \mathrm{ft}$ near the side of the river valley to as much as $47 \mathrm{ft}$ near the center of the valley. The use of drilling fluid also precluded the determination of ground-water levels in these holes during drilling.

The drillhole data indicate that the alluvium probably is of minor importance as an aquifer. It is relatively thin, limited in areal extent, and probably not fully saturated.

Consolidated rocks contain ground water in much of the study area as indicated by the presence of springs and by data from wells and drilling during this study; however, these rocks may not be fully saturated near incised canyons or on terraces. Most of the ground water in consolidated rocks is in joints and fractures or in the intergranular space in weakly cemented sandstone (Cordova, 1981, p. 16).

The ground-water system in consolidated rocks in the study area includes a regional water-table aquifer at depth in the Navajo Sandstone (Gates, 1965) overlain by saturated zones in many of the formations that overlie the Navajo Sandstone. The extent of these saturated zones, both laterally and vertically, is relatively unknown because of limited data. Some of the saturated zones may be perched where they are unoonfined and separated from an underlying body of ground water by an unsaturated zone. For example, ground water in the Termle Cap Sandstone is perched because several hundred feet of unsaturated rock separates this saturated zone from the underlying regional water table in the Navajo Sandstone. Saturated zones in the Tropic Shale and Carmel Formation may be of limited extent and thus, could be oonsidered local aquifers. The abundance of springs in the Straight Cliffs Sandstone indicates that this formation may have a substantial saturated zone and be an aquifer of regional extent.

The basal saturated part of the Navajo Sandstone constitutes the Navajo aquifer, the regional water-table aquifer. It is a principal aquifer in the area because of the large number of springs and several wells to which it provides water. The water table in the Navajo aquifer is at a depth of about $870 \mathrm{ft}$ (elevation 4,830 ft) at well w8 (Gates, 1965, p. 157) (fig. 14). The zone of springs at the base of the Navajo Sandstone, 2 mi north of Springdale 
(fig. 1), which supply Zion National Park Headquarters, probably mark the intersection of the regional water table in the Navajo aquifer with the walls of Zion Canyon (Gates, 1965, p. 156).

Very little information is available on the Navajo aquifer in this area because of the lack of wells and its depth. In the coal-lease tract, the top of the Navajo Sandstone is more than $985 \mathrm{ft}$ deep at well LCl, and the depth increases to the east-northeast as the overburden thickness increases downdip.

An aquifer at the base of the Temple Cap Sandstone was penetrated by a drillhole at site LC2 (pl. 2). The hole was drilled to a depth of $359 \mathrm{ft}$ (table 7), and ground water was first encountered at a depth of about $130 \mathrm{ft}$ in the Temple Cap Sandstone. Of two piezometers installed in the drillhole, the first was screened from 344 to $354 \mathrm{ft}$ in the Navajo Sandstone, but was dry. The open-ended casing of the second piezometer extended to a depth of $147 \mathrm{ft}$ and reflected the water level in the open hole from 0 to $172 \mathrm{ft}$. The water level was about $120 \mathrm{ft}$ below land surface in the shallower piezometer. During drilling, this shallow aquifer, the Temple Cap, produced about 30 $\mathrm{gal} / \mathrm{min}$ from a depth of about $150 \mathrm{ft}$. The Temple Cap aquifer is above a 14-ft thick, brick-red siltstone that marks its base at this location. Below the siltstone, to a depth of at least $359 \mathrm{ft}$, the underlying Navajo Sandstone appears to be unsaturated.

West of the lease tract, subsurface information on the occurrence of ground water in the Temple Cap aquifer is available from investigations in 1976 and 1980 for a proposed dam on the North Fork Virgin River (Bingham Engineering, 1980). Twenty-two drillholes, both angled and vertical, were drilled in the vicinity of well LC2 and about $0.5 \mathrm{mi}$ downstream (T. $39 \mathrm{~S}$., R. 9 W. , sec. 32, SW $\left.\frac{1}{4} S W_{\frac{1}{4}} S W^{\frac{1}{4}}\right)$. The 14 drillholes near well LC2 ranged in depth from 9 to $142.3 \mathrm{ft}$, and the 8 drillholes downstream ranged in depth from 22.5 to $121 \mathrm{ft}$. Ground water was present in only one of these drillholes penetrating the Temple Cap aquifer, about $900 \mathrm{ft}$ west of LC2 on the north side of the river. The water level in this 70-ft deep drillhole, following drilling in May 1980, was at a depth of $54 \mathrm{ft}$; however, by October 1980, the depth to water was more than $63 \mathrm{ft}$. The May water level, taken 4 days after drilling, may actually represent drilling fluid, which infiltrated into the surrounding formation by the time the october measurement was made.

The Temple Cap aquifer also is penetrated by domestic wells W5, W6, and W7 (fig. 14). The spring at site S39 (fig. 14) also issues from a saturated zone, probably the Temple Cap aquifer.

Three water-bearing zones are present in the Carmel Formation, coinciding with parts of the Winsor Member and the banded and limestone members, as evidenced by spring locations and drilling during this investigation. Four monitoring wells were completed in aquifers of the Carmel Formation (table 7). During air rotary drilling at site $\mathrm{LCl}$, ground water was detected about $35 \mathrm{ft}$ below land surface at the base of the coal-bearing member of the Tropic Shale. Flow into the drillhole increased noticeably at a depth of about $65 \mathrm{ft}$ during drilling in the Winsor aquifer. The flow increased from about $2.7 \mathrm{gal} / \mathrm{min}$ at a depth of $70 \mathrm{ft}$ to $4.3 \mathrm{gal} / \mathrm{min}$ at $75 \mathrm{ft}$, decreasing again to about $3 \mathrm{gal} / \mathrm{min}$ at a depth of $115 \mathrm{ft}$. On October 15, 1987, a piezometer installed at a depth of about $580 \mathrm{ft}$ in the banded aquifer in the Carmel Formation (LCl, table 7), 


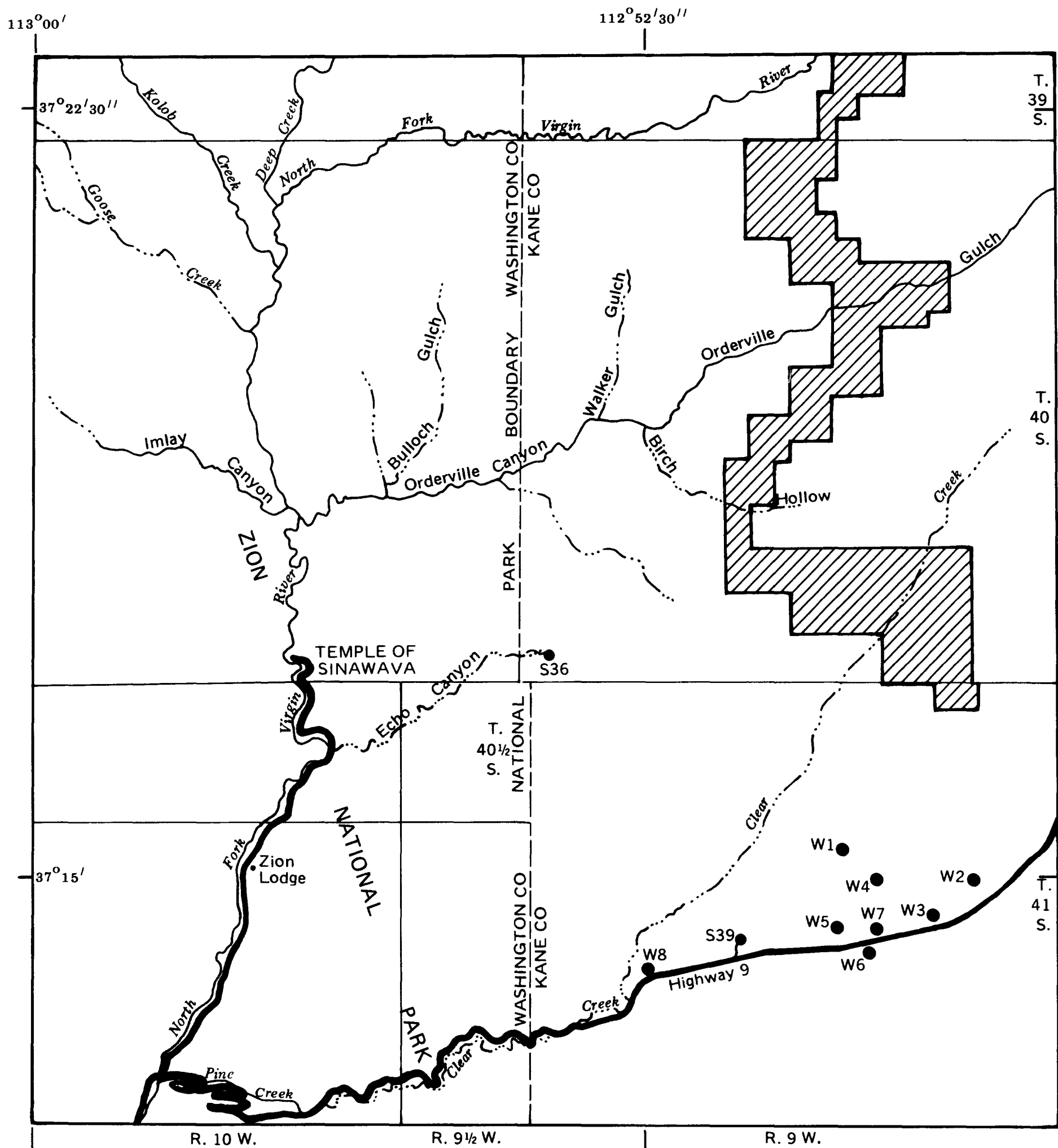

Base from U.S. Geological Survey $1: 100,000$ quadrangle: Kanab, Utah, 1980

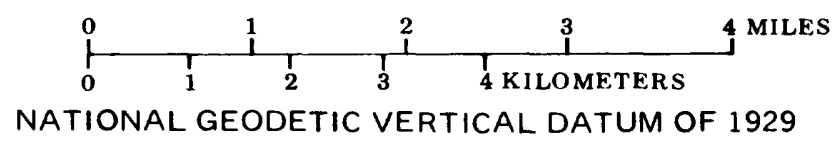

Figure 14.--Location of and water-quality diagrams for wells and springs outside the L.C. Holding coal-lease tract. 

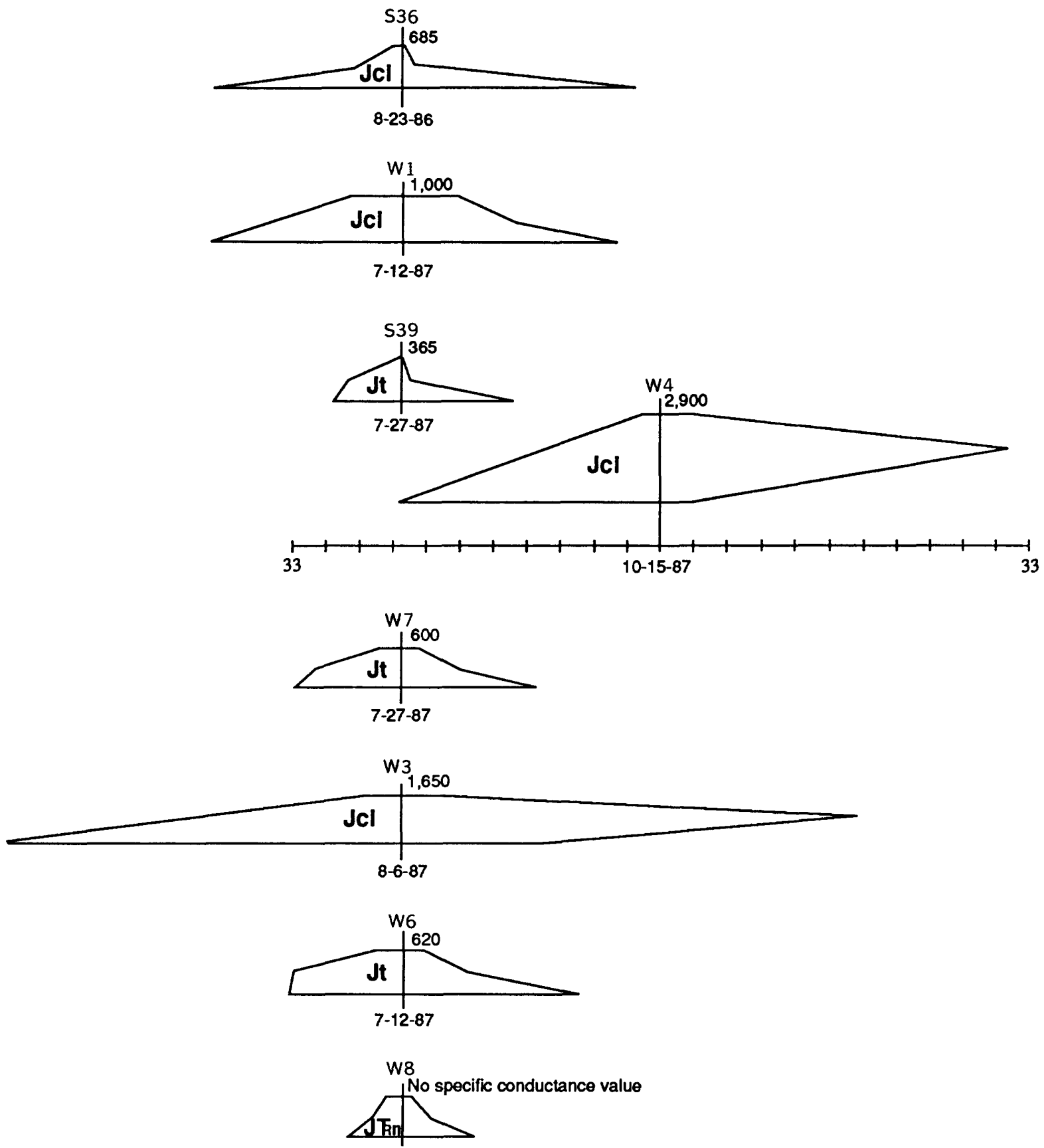

7-6-62

EXPLANATION

L.C. HOLDING COAL-LEASE TRACT

S36 SPRINGAND IDENTIFIER

W6 WELLAND IDENTIFIER

Geologic formations: JCl, limestone member of Carmel Formation;

It, Temple Cap Sandatone; Jhn, Navajo Sandistone

\section{WATER-QUALITY DIAGRAM}

(Modified from StIff, 1951)

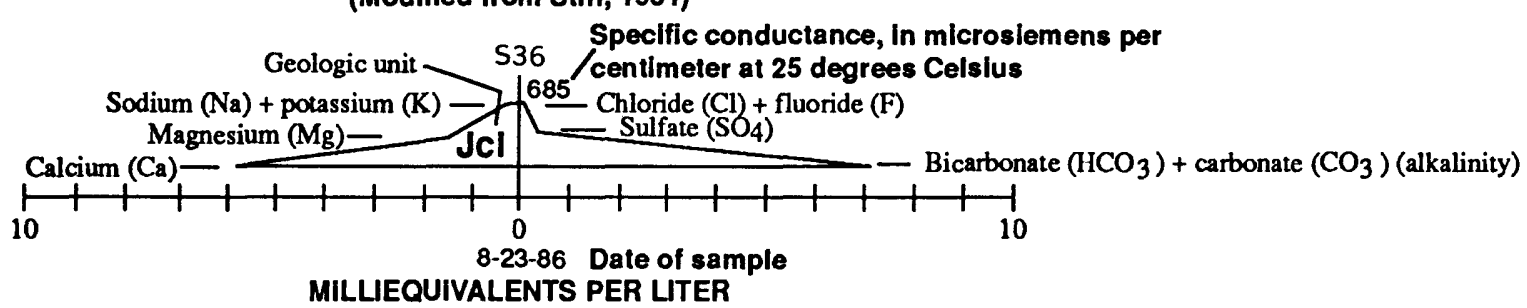

MILLIEQUIVALENTS PER LITER

Figure 14.-- Location of and water-quality diagrams for wells and springs outside the L.C. Holding coal-lease tract--Continued. 
indicated a hydraulic head $228 \mathrm{ft}$ below that of the head measured in the Winsor aquifer at $\mathrm{LCl}$. This head difference indicates a downward vertical head gradient in this area.

A spring, S14 (pl. 1), which flows through a solution cavity in a fallen block of the gypsiferous member of the Carmel Formation, issues from the banded aquifer. Discharge from this spring is about $20 \mathrm{gal} / \mathrm{min}$, a relatively large flow for springs in the study area. During this study, no other springs were observed discharging from the banded aquifer.

Two wells at LC3 were completed in the limestone aquifer in the Carmel Formation. During drilling, ground water entered the drillhole at $35 \mathrm{ft}$ at a rate of less than $1 \mathrm{gal} / \mathrm{min}$. The rate of inflow increased to $25 \mathrm{gal} / \mathrm{min}$ at 75 $\mathrm{ft}$ and about $200 \mathrm{gal} / \mathrm{min}$ at $136 \mathrm{ft}$. A loss of circulation and increased water production indicated that open fractures or solution cavities were intercepted at a depth of $136 \mathrm{ft}$. Water levels in the two wells at LC3 show a difference in hydraulic head of about $23 \mathrm{ft}$. The higher head is in the well that is open to strata from 0 to $115 \mathrm{ft}$ below land surface, and the lower head is in the well perforated from 124 to $157 \mathrm{ft}$. This indicates a downward vertical head gradient.

The limestone aquifer in the Carmel Formation is the source of water for four domestic wells ( $W 1, W 2, W 3$, and $W 4$ ) south of the lease tract (fig. 14). Well $W 3$ is no longer in use. Springs discharging fram the limestone aquifer include S36 (fig. 14) and several seeps from the limestone cliff adjacent to well LC3 (pl. 2).

Well LC4 (pl. 2) was completed in the basal part of the coal-bearing member of the Tropic Shale and penetrated the basal coal that could be mined by surface methods. The water level for the water-bearing coal and overlying sandstone (see log in "Supplemental Information" at end of report) is more that $100 \mathrm{ft}$ above the perforated zone.

Only five springs were found to discharge from aquifers in the Tropic Shale, and all but one of these springs issued from the upper shaley member. However, numerous seeps were noted in the area of S13 issuing fram the aquifer in the coal zone near the base of the coal-bearing member of the Tropic Shale.

The Straight $\mathrm{Cliffs}$ Sandstone contains ground water as indicated by the 25 springs that discharge from this formation within the study area (pl. 1). Three springs in the Straight $\mathrm{Cliffs}$ aquifer have discharges greater than 50 $\mathrm{gal} / \mathrm{min}$ (table 8 ; S12, S23, S32). Seepage from canyon walls takes place at the top of shale lenses in the straight Cliffs aquifer. These areas of seepage were noted in Little Meadow Creek canyon (S21), Willow Canyon (S3), and in alluvium overlying the Straight Cliffs Sandstone in Orderville Gulch and Hay Canyon (pl. 1).

Five springs discharge from the Wahweap Sandstone and Kaiparowits Formation (S1, S4, S7, S8, and S9). The interbedded sandstone and shale typical of the Wahweap and Kaiparowits units form aquifers (sandstone) and confining layers (shale). 
The hydrologic characteristics of the basic extrusive rocks (Qb), which crop out in the upper canyon of the North Fork Virgin River and in the northwest corner of the study area (pl. 1), are unknown. They probably yield little, if any, water and may be a barrier to ground-water movement in places; however, they are highly permeable where they are fractured or contain lava tubes or vesicular surfaces along the boundaries between lava flows (Wilson and Thomas, 1964, p. C19).

\section{Aquifer Characteristics}

Data on aquifer characteristics are lacking for most of the aquifers in the study area because of the lack of wells and aquifer tests; however, more data are available for the Temple Cap and Navajo aquifers than for other aquifers. The specific capacity of well W7, completed in the Temple Cap aquifer, is 0.5 (gal/min)/ft (Cordova, 1981, p. 27). For the Navajo aquifer in the upper Virgin River and Kanab Creek basins, Cordova (1981, p. 23) reported a range of values for specific capacity from 0.03 to $23(\mathrm{gal} / \mathrm{min}) / \mathrm{ft}$.

In a study of the Alton coal field, east of the L.C. Holding coal-lease tract, Freethey (1988, p. 16) reported that the specific-capacity values for wells in fractured Navajo Sandstone were larger than values for unfractured sandstone. Wells within 2,000 ft of a fault zone had specific-capacity values of 0.7 to 23 (gal/min)/ft; whereas, wells more than $2,000 \mathrm{ft}$ from a fault zone had specific-capacity values of 0.03 to 2.9 (gal/min)/ft.

From two rock samples of the Navajo Sandstone $\infty 1$ lected on the east side of Zion National Park, Uygur (1980, p. 39) determined horizontal-hydraulic conductivity values of 1.8 and $3.3 \mathrm{ft} /$ day and vertical hydraulic onductivity values of 0.32 and $0.87 \mathrm{ft} /$ day. Freethey $(1988, \mathrm{p}$. 16) reported that horizontal conductivity in the Navajo aquifer is generally about 2.5 times larger than vertical conductivity on the basis of data from previous studies in the Alton coal field and the upper Virgin River and Kanab Creek basins.

In the central Virgin River basin, west of $\mathrm{Zion}$ National Park, the average horizontal hydraulic conductivity from rock samples of the Navajo Sandstone was $2.1 \mathrm{ft} /$ day (Cordova, 1978, p. 26). An average hydraulic conductivity of $5 \mathrm{ft} /$ day was determined from aquifer tests in the same area (Cordova, 1978, p. 27). The conductivity value from the aquifer tests is considered a more reasonable estimate for the local area because aquifer tests include rock containing fractures; whereas, core samples represent unfractured rock, and resulting hydraulic-conductivity values are less than those from aquifer tests. Values for horizontal hydraulic conductivity, 2.4 and 7.5 $\mathrm{ft} /$ day, were reported from Navajo aquifer tests in the Alton coal field, and faulting was a factor in the area of testing (Freethey, 1988, p. 18).

Gates (1965, p. 158) described a bailing/drawdown test at well w8 (fig. 14 ), which provided an estimated transmissivity of $1,070 \mathrm{ft}^{2} /$ day for the Navajo aquifer (reported as coefficient of transmissibility of 8,000 gal/day/ft). Cordova (1981, p. 22) reported transmissivity values ranging from 2,500 to $14,000 \mathrm{ft}^{2} /$ day from aquifer tests of the Navajo aquifer in the upper Virgin River and Kanab Creek basins. He noted that the larger values probably reflected the full saturated thickness (as much as 2,000 ft) of the Navajo aquifer. 
[Al] $\mathrm{pH}$, specific conductance, and alkalinity values were obtained in the field Discharge values marked with an ' $E$ ' are estimated; ${ }^{\circ} \mathrm{C}$, degrees Celsius;

$\mathrm{mg} / \mathrm{L}$, miliigrams per liter;

Site identifier: Locations show on plate 1 and figures 9 and 14.

Geologic unit: Qal, alluvium; Kwk, Wahweap Sandstone and Kaiparowits Formation; Ksc, Straight Cliffs member of Carmel Formation; Jcl, limestone member of Carmel Formation; Jt, Temple Cap Sandstone; JTm,

\begin{tabular}{|c|c|c|c|c|c|c|c|c|c|}
\hline $\begin{array}{l}\text { Site } \\
\text { ident- } \\
\text { ifier }\end{array}$ & Location & $\begin{array}{c}\text { Geologic } \\
\text { unit }\end{array}$ & $\begin{array}{l}\text { Discharge } \\
\text { (gallons } \\
\text { per minute) }\end{array}$ & Date & $\begin{array}{l}\text { Temper- } \\
\text { ature } \\
\left({ }^{\circ} \mathrm{C}\right)\end{array}$ & $\begin{array}{l}\text { Spe- } \\
\text { cific } \\
\text { con- } \\
\text { duct- } \\
\text { ance } \\
(\mu S / \mathrm{cm})\end{array}$ & $\begin{array}{l}\text { pH } \\
\text { (stand- } \\
\text { and } \\
\text { units) }\end{array}$ & $\begin{array}{l}\text { Calcium, } \\
\text { dis- } \\
\text { solved } \\
\text { (mg/L } \\
\text { as Ca) }\end{array}$ & $\begin{array}{l}\text { Magne- } \\
\text { sium, } \\
\text { dis- } \\
\text { solved } \\
\text { (mg/L } / \mathrm{L} \\
\text { as } \mathrm{Mg} \text { ) }\end{array}$ \\
\hline
\end{tabular}

\begin{tabular}{|c|c|c|c|c|c|c|c|c|c|}
\hline \multicolumn{10}{|c|}{ SPRINGS } \\
\hline $\begin{array}{l}\text { \$1 } \\
\text { S2 } \\
\text { S3 } \\
\text { S4 } \\
\text { S5 } \\
\text { S6 }\end{array}$ & $\begin{array}{r}\text { (C-39- 8) } 5 \mathrm{abd}-S 1 \\
18 \mathrm{dcd}-S 1 \\
19 \mathrm{cca}-S 1 \\
20 \mathrm{bab}-S 1 \\
20 \mathrm{cb}-S 1 \\
29 \mathrm{cda}-S 1\end{array}$ & $\begin{array}{l}\text { Kwk } \\
\text { Ksc } \\
\text { Ksc } \\
\text { Kwk } \\
\text { Ksc } \\
\text { Qal }\end{array}$ & $\begin{array}{l}66 \\
0.2 \\
1.3 \\
4.0 \\
1.6 \\
0.3\end{array}$ & $\begin{array}{l}09-13-86 \\
08-24-86 \\
09-10-86 \\
08-24-86 \\
08-24-86 \\
09-12-86\end{array}$ & $\begin{array}{r}9.5 \\
8.5 \\
11.0 \\
14.0 \\
12.0 \\
13.0\end{array}$ & $\begin{array}{r}740 \\
1,090 \\
1,700 \\
1,200 \\
1,530 \\
675\end{array}$ & $\begin{array}{l}7.2 \\
7.2 \\
7.6 \\
8.8 \\
7.9 \\
7.2\end{array}$ & $\begin{array}{r}100 \\
200 \\
180 \\
190 \\
110\end{array}$ & $\begin{array}{r}46 \\
-- \\
140 \\
62 \\
110 \\
27\end{array}$ \\
\hline $\begin{array}{l}\text { S7 } \\
\text { S8 } \\
\text { S9 } \\
\text { S10 } \\
\text { S11 }\end{array}$ & $\begin{array}{r}(C-39-9) 10 \mathrm{ddc}-\$ 1 \\
16 \mathrm{abd}-S 1 \\
16 \mathrm{bac}-\$ 1 \\
16 \mathrm{dcc}-S 1 \\
22 \mathrm{cba}-S 1\end{array}$ & $\begin{array}{l}\text { Kwk } \\
\text { Kwk } \\
\text { Kwk } \\
\text { Ksc } \\
\text { Kts }\end{array}$ & $\begin{array}{l}3.9 \\
0.25 \\
0.9 \\
1.2 \\
9.6\end{array}$ & $\begin{array}{l}08-26-86 \\
09-08-86 \\
09-08-86 \\
09-11-86 \\
08-23-86\end{array}$ & $\begin{array}{r}8.0 \\
15.0 \\
14.0 \\
8.5 \\
11.5\end{array}$ & $\begin{array}{l}650 \\
520 \\
450 \\
580 \\
560\end{array}$ & $\begin{array}{l}7.2 \\
7.3 \\
7.4 \\
8.3 \\
7.4\end{array}$ & $\begin{array}{r}110 \\
-- \\
74 \\
78 \\
68\end{array}$ & $\begin{array}{l}25 \\
-- \\
11 \\
31 \\
27\end{array}$ \\
\hline $\begin{array}{l}\text { S12 } \\
\text { S13 } \\
\text { S14 } \\
\text { S15 }\end{array}$ & $\begin{array}{l}23 a b c-\$ 1 \\
24 b a d-S 1 \\
33 a d a-S 1 \\
36 a b d-S 1\end{array}$ & $\begin{array}{l}\text { Ksc } \\
\text { Ktc } \\
\text { Jcb } \\
\text { Ksc }\end{array}$ & $\begin{array}{c}280 \\
0.13 \\
20 \mathrm{E} \\
3.1\end{array}$ & $\begin{array}{l}08-25-86 \\
09-17-87 \\
07-28-87 \\
07-17-86\end{array}$ & $\begin{array}{r}11.0 \\
9.5 \\
11.0 \\
7.5\end{array}$ & $\begin{array}{r}450 \\
1,230 \\
2,450 \\
840\end{array}$ & $\begin{array}{l}7.6 \\
8.7 \\
7.2 \\
7.4\end{array}$ & $\begin{array}{r}60 \\
-- \\
320 \\
110\end{array}$ & $\begin{array}{l}23 \\
\overline{36} \\
46\end{array}$ \\
\hline $\begin{array}{l}\text { \$16 } \\
\text { S17 } \\
\text { S18 } \\
\text { \$19 }\end{array}$ & $\begin{array}{r}(C-40-8) \\
5 \mathrm{ccd}-S 1 \\
7 \mathrm{aad}-S 1 \\
7 \mathrm{cad}-S 1 \\
7 \mathrm{cda}-S 1\end{array}$ & $\begin{array}{l}\text { Ksc } \\
\text { KsC } \\
\text { KsC } \\
\text { KSC }\end{array}$ & $\begin{array}{l}4.4 \\
5.0 \\
2.4 \\
1.1\end{array}$ & $\begin{array}{l}06-24-86 \\
07-13-86 \\
07-12-86 \\
07-12-86\end{array}$ & $\begin{array}{r}16.0 \\
9.0 \\
8.0 \\
7.5\end{array}$ & $\begin{array}{l}710 \\
560 \\
520 \\
460\end{array}$ & $\begin{array}{l}7.9 \\
7.9 \\
7.7 \\
7.7\end{array}$ & $\begin{array}{r}100 \\
74 \\
-- \\
68\end{array}$ & $\begin{array}{l}36 \\
29 \\
-\overline{17}\end{array}$ \\
\hline $\begin{array}{l}\$ 20 \\
\$ 21 \\
\$ 22\end{array}$ & $\begin{array}{l}29 b c a-\$ 1 \\
30 \mathrm{bad}-\$ 1 \\
32 \mathrm{dbd}-\$ 1\end{array}$ & $\begin{array}{l}\text { Kts } \\
\text { Ksc } \\
\text { Kts }\end{array}$ & $\begin{array}{l}8.3 \\
15 \\
8.6 \\
8.6 \\
0.7\end{array}$ & $\begin{array}{l}08-28-81 \\
08-19-86 \\
08-28-81 \\
08-20-86 \\
08-19-86\end{array}$ & $\begin{array}{r}9.0 \\
10.0 \\
9.0 \\
9.5 \\
19.0\end{array}$ & $\begin{array}{r}1,080 \\
850 \\
695 \\
570 \\
1,550\end{array}$ & $\begin{array}{l}7.0 \\
7.1 \\
7.3 \\
7.4 \\
7.4\end{array}$ & $\begin{array}{r}110 \\
100 \\
82 \\
83 \\
89\end{array}$ & $\begin{array}{l}52 \\
49 \\
26 \\
26 \\
39\end{array}$ \\
\hline $\begin{array}{l}\text { S23 } \\
\text { S24 } \\
\text { S25 }\end{array}$ & $\begin{array}{r}(C-40-9) 12 \text { daa-S1 } \\
\text { 12daa-S2 } \\
12 \text { dad-S1 }\end{array}$ & $\begin{array}{l}\text { KsC } \\
\text { KSC }\end{array}$ & $\begin{array}{l}60 \\
60 \\
60 \\
28 \\
7.5\end{array}$ & $\begin{array}{l}07-13-86 \\
08-22-86 \\
09-09-86 \\
07-13-86 \\
07-12-86\end{array}$ & $\begin{array}{r}11.5 \\
11.5 \\
12.0 \\
11.5 \\
8.5\end{array}$ & $\begin{array}{l}610 \\
600 \\
600 \\
675 \\
540\end{array}$ & $\begin{array}{l}7.4 \\
7- \\
7.4 \\
7.2 \\
7.6\end{array}$ & $\begin{array}{l}79 \\
-- \\
-- \\
-- \\
--\end{array}$ & $\begin{array}{l}28 \\
-- \\
-- \\
-- \\
--\end{array}$ \\
\hline $\begin{array}{l}\$ 26 \\
\$ 27 \\
\$ 28 \\
\$ 29 \\
\text { S30 }\end{array}$ & $\begin{array}{r}(C-40-9) 13 b d c-S 1 \\
22 c d c-S 1 \\
23 b c c-S 1 \\
26 b b c-S 1 \\
26 b c b-S 1\end{array}$ & $\begin{array}{l}\text { Ksc } \\
\text { Kts } \\
\text { Ksc } \\
\text { Ksc } \\
\text { KsC }\end{array}$ & $\begin{array}{l}1.7 \\
13 \\
7.5 \\
5.7 \\
9.2\end{array}$ & $\begin{array}{l}08-22-86 \\
07-15-86 \\
08-21-86 \\
08-21-86 \\
08-21-86\end{array}$ & $\begin{array}{l}7.0 \\
8.0 \\
8.0 \\
9.0 \\
8.0\end{array}$ & $\begin{array}{l}980 \\
490 \\
590 \\
350 \\
335\end{array}$ & $\begin{array}{l}7.6 \\
7.6 \\
7.8 \\
7.7 \\
7.6\end{array}$ & $\begin{array}{r}120 \\
70 \\
75 \\
64 \\
--\end{array}$ & $\begin{array}{l}62 \\
26 \\
28 \\
10 \\
--\end{array}$ \\
\hline $\begin{array}{l}\text { S31 } \\
\text { S32 }\end{array}$ & $\begin{array}{l}26 c a a-\$ 1 \\
26 c d a-\$ 1 \\
26 c d c-\$ 1\end{array}$ & $\begin{array}{l}\text { Ksc } \\
\text { KsC }\end{array}$ & $\begin{array}{c}9.3 \\
80 \\
100 \mathrm{E} \\
61 \\
6.0 \\
5.8 \\
6.0\end{array}$ & $\begin{array}{l}09-13-86 \\
08-28-81 \\
06-07-82 \\
09-14-86 \\
08-28-81 \\
06-07-82 \\
08-21-86\end{array}$ & $\begin{array}{r}9.0 \\
9.0 \\
10.0 \\
10.0 \\
10.0 \\
10.5 \\
11.0\end{array}$ & $\begin{array}{l}490 \\
475 \\
510 \\
495 \\
510 \\
530 \\
470\end{array}$ & $\begin{array}{l}7.5 \\
7.3 \\
7.4 \\
7.5 \\
7.3 \\
7.6 \\
7.5\end{array}$ & $\begin{array}{l}-- \\
93 \\
74 \\
77 \\
72 \\
72 \\
--\end{array}$ & $\begin{array}{l}-- \\
28 \\
20 \\
19 \\
23 \\
23 \\
--\end{array}$ \\
\hline
\end{tabular}


of water from selected springs and wells

except those marked ' $L$ ', which were measured in the laboratory.

$\mathrm{S} / \mathrm{cm}$, microsiemens per cent imeters at 25 degrees Celsius;

$\mu \mathrm{g} / \mathrm{L}$, micrograms per liter]

Sandstone; Kts, shaly member of Tropic Shale; Ktc, coal-bearing member of Tropic Shale; Jcb, banded Navajo Sandstone.

\begin{tabular}{|c|c|c|c|c|c|c|c|c|c|c|c|}
\hline $\begin{array}{l}\text { Sodium, } \\
\text { dis- } \\
\text { solved } \\
\text { (mg/L } \\
\text { as } \mathrm{Na} \text { ) }\end{array}$ & $\begin{array}{l}\text { Potas- } \\
\text { sium, } \\
\text { dis- } \\
\text { solved } \\
\text { (mg/L } \\
\text { as K) }\end{array}$ & $\begin{array}{l}\text { Alka- } \\
\text { linity, } \\
\text { total } \\
\text { field } \\
(\mathrm{mg} / \mathrm{L} \text { as } \\
\left.\mathrm{CaO}_{3}\right)\end{array}$ & $\begin{array}{l}\text { Sulfate, } \\
\text { dis- } \\
\text { solved } \\
\text { (mg/L } \\
\text { as } \mathrm{SO}_{4} \text { ) }\end{array}$ & $\begin{array}{l}\text { Chlo- } \\
\text { ride, } \\
\text { dis- } \\
\text { solved } \\
\text { (mg/L } \\
\text { as Cl) }\end{array}$ & $\begin{array}{l}\text { Fluo- } \\
\text { ride, } \\
\text { dis- } \\
\text { solved } \\
\text { (mg/L } \\
\text { as } F \text { ) }\end{array}$ & $\begin{array}{c}\text { Silica, } \\
\text { dis- } \\
\text { solved } \\
\text { (mg/L } \\
\text { as } \\
\mathrm{SiO}_{2} \text { ) }\end{array}$ & $\begin{array}{l}\text { Solids, } \\
\text { residue } \\
\text { at } 180 \\
{ }^{\circ} \mathrm{C}, \\
\text { dis- } \\
\text { solved } \\
(\mathrm{mg} / \mathrm{L})\end{array}$ & $\begin{array}{l}\text { Solids, } \\
\text { sum of } \\
\text { consti- } \\
\text { tuents, } \\
\text { dis- } \\
\text { solved } \\
\text { (mg/L) }\end{array}$ & $\begin{array}{l}\text { Hard- } \\
\text { ness } \\
(\mathrm{mg} / \mathrm{L} \\
\text { as } \\
\left.\mathrm{CaCO}_{3}\right)\end{array}$ & $\begin{array}{l}\text { Manga- } \\
\text { nese, } \\
\text { dis- } \\
\text { solved } \\
(\mu \mathrm{g} / \mathrm{L} \\
\text { as } \mathrm{Mn})\end{array}$ & $\begin{array}{r}\text { Iron, } \\
\text { dis- } \\
\text { solved } \\
\text { ( } \mu \text { g/L } \\
\text { as Fe) }\end{array}$ \\
\hline \multicolumn{12}{|c|}{ SPRINGS } \\
\hline 2.8 & 1.7 & 396 & 37 & 2.8 & 0.2 & 9.5 & 333 & 440 & 440 & 4 & 10 \\
\hline $\begin{array}{c}25 \\
4.6 \\
7.1 \\
3.2\end{array}$ & $\begin{array}{l}-- \\
3.5 \\
3.0 \\
5.9 \\
1.9\end{array}$ & $\begin{array}{l}500 \\
390 \\
369 \\
282\end{array}$ & $\begin{array}{r}550 \\
320 \\
570 \\
94\end{array}$ & $\begin{array}{l}\overline{16} \\
5.7 \\
6.9 \\
4.1\end{array}$ & $\begin{array}{l}\overline{0.4} \\
0.4 \\
0.6 \\
0.4\end{array}$ & $\begin{array}{c}16 \\
14 \\
9.9 \\
12\end{array}$ & $\begin{array}{r}1, \overline{160} \\
700 \\
1,140 \\
430\end{array}$ & $\begin{array}{r}1, \overline{300} \\
820 \\
1,100 \\
420\end{array}$ & $\begin{array}{r}1, \overline{100} \\
700 \\
930 \\
390\end{array}$ & $\begin{array}{l}36 \\
580 \\
120 \\
170\end{array}$ & $\begin{array}{r}\overline{22} \\
8 \\
7 \\
1,400\end{array}$ \\
\hline 2.6 & 1.4 & 291 & 70 & 2.7 & 0.2 & 9.0 & 333 & 400 & 380 & 55 & 560 \\
\hline $\begin{array}{r}2.5 \\
3.6 \\
12\end{array}$ & $\begin{array}{l}0.9 \\
2.7 \\
2.9\end{array}$ & $\begin{array}{l}207 \\
217 \\
255\end{array}$ & $\begin{array}{l}25 \\
94 \\
54\end{array}$ & $\begin{array}{l}5.3 \\
3.9 \\
4.0\end{array}$ & $\begin{array}{r}0.2 \\
0.2 \\
<0.1\end{array}$ & $\begin{array}{r}11 \\
8.0 \\
8.8\end{array}$ & $\begin{array}{l}264 \\
335 \\
310\end{array}$ & $\begin{array}{l}250 \\
350 \\
330\end{array}$ & $\begin{array}{l}230 \\
320 \\
280\end{array}$ & $\begin{array}{r}4 \\
<1 \\
2\end{array}$ & $\begin{array}{r}30 \\
5 \\
<3\end{array}$ \\
\hline 3.6 & 1.6 & 201 & 40 & 1.9 & 0.1 & 9.6 & 248 & 260 & 240 & $<1$ & $<3$ \\
\hline 27 & $\begin{array}{l}2.5 \\
2.2\end{array}$ & $\begin{array}{l}1 \overline{8} \\
314\end{array}$ & $\begin{array}{r}1,400 \\
170\end{array}$ & $\begin{array}{l}13 \\
4.6\end{array}$ & $\begin{array}{l}0.3 \\
0.2\end{array}$ & $\begin{array}{c}1 \overline{-} \\
8.2\end{array}$ & $2, \overline{360}$ & $\begin{array}{r}1, \overline{900} \\
540\end{array}$ & $\begin{array}{l}950 \\
460\end{array}$ & $\begin{array}{l}\overline{10} \\
<1\end{array}$ & $\begin{array}{r}30 \\
8\end{array}$ \\
\hline $\begin{array}{l}4.5 \\
2.8\end{array}$ & $\begin{array}{l}2.2 \\
1.8\end{array}$ & $\begin{array}{l}411 \\
280\end{array}$ & $\begin{array}{l}73 \\
35\end{array}$ & $\begin{array}{l}3.6 \\
2.9\end{array}$ & $\begin{array}{l}0.2 \\
0.2\end{array}$ & $\begin{array}{l}9.6 \\
9.7\end{array}$ & $\overline{262}$ & $\begin{array}{l}480 \\
320\end{array}$ & $\begin{array}{l}400 \\
300\end{array}$ & $\begin{array}{r}150 \\
6\end{array}$ & $\begin{array}{l}9 \\
5\end{array}$ \\
\hline$\overline{1.9}$ & $\overline{2.0}$ & $\begin{array}{l}295 \\
248\end{array}$ & $\overline{16}$ & $\overline{1.7}$ & $\overline{0.2}$ & $\overline{7.3}$ & $\overline{211}$ & $\overline{260}$ & $\overline{240}$ & $\overline{<1}$ & 6 \\
\hline $\begin{array}{r}9.5 \\
9.3 \\
2.3 \\
2.2 \\
230\end{array}$ & $\begin{array}{l}3.4 \\
3.3 \\
2.1 \\
2.0 \\
5.6\end{array}$ & $\begin{array}{l}220 \mathrm{~L} \\
344 \\
150 \mathrm{~L} \\
288 \\
392\end{array}$ & $\begin{array}{r}180 \\
120 \\
38 \\
35 \\
500\end{array}$ & $\begin{array}{c}5.4 \\
4.0 \\
10 \\
2.1 \\
11\end{array}$ & $\begin{array}{l}0.2 \\
0.2 \\
0.1 \\
0.1 \\
0.3\end{array}$ & $\begin{array}{c}10 \\
9.9 \\
9.1 \\
8.8 \\
15\end{array}$ & $\begin{array}{c}-- \\
480 \\
-- \\
320 \\
1,180\end{array}$ & $\begin{array}{r}500 \\
500 \\
260 \\
330 \\
1,100\end{array}$ & $\begin{array}{l}480 \\
450 \\
310 \\
310 \\
380\end{array}$ & $\begin{array}{r}1 \\
<1 \\
2 \\
1 \\
8\end{array}$ & $\begin{array}{r}<10 \\
9 \\
<10 \\
6 \\
8\end{array}$ \\
\hline 4.6 & 1.6 & 276 & 54 & 2.6 & 0.1 & 8.3 & 347 & 340 & 310 & $<1$ & 6 \\
\hline$=$ & $\overline{-}$ & $\overline{-}$ & $=$ & $=$ & $=$ & $\overline{-}$ & -- & $=$ & -- & $\begin{array}{l}-- \\
-\end{array}$ & $\begin{array}{l}-- \\
--\end{array}$ \\
\hline -- & -- & $\bar{m}$ & -- & -- & -- & -- & -- & -- & -- & -- & -- \\
\hline- & -- & 290 & -- & -- & -- & -- & -- & -- & -- & -- & -- \\
\hline $\begin{array}{l}3.9 \\
4.2 \\
3.3 \\
1.2\end{array}$ & $\begin{array}{l}2.7 \\
1.8 \\
1.4 \\
0.5\end{array}$ & $\begin{array}{l}324 \\
268 \\
280 \\
203\end{array}$ & $\begin{array}{c}230 \\
40 \\
40 \\
4.0\end{array}$ & $\begin{array}{l}3.6 \\
3.1 \\
2.5 \\
1.2\end{array}$ & $\begin{array}{l}0.2 \\
0.1 \\
0.1 \\
0.1\end{array}$ & $\begin{array}{l}9.8 \\
7.7 \\
8.2 \\
7.3\end{array}$ & $\begin{array}{l}730 \\
273 \\
305 \\
216\end{array}$ & $\begin{array}{l}630 \\
310 \\
330 \\
210\end{array}$ & $\begin{array}{l}550 \\
280 \\
300 \\
200\end{array}$ & $\begin{array}{r}5 \\
<1 \\
2 \\
<1\end{array}$ & $\begin{array}{l}5 \\
6 \\
8 \\
6\end{array}$ \\
\hline-- & - & -- & - & -- & -- & - & -- & -- & -- & -- & -- \\
\hline-- & - & -- & -- & -- & -- & -- & -- & -- & -- & -- & -- \\
\hline $\begin{array}{l}3.7 \\
1.9 \\
1.9 \\
3.0 \\
2.8\end{array}$ & $\begin{array}{l}1.4 \\
1.0 \\
0.9 \\
1.5 \\
1.6\end{array}$ & $\begin{array}{l}150 \mathrm{~L} \\
250 \\
250 \\
150 \mathrm{~L} \\
250\end{array}$ & $\begin{array}{l}62 \\
24 \\
23 \\
38 \\
36\end{array}$ & $\begin{array}{l}3.3 \\
2.0 \\
2.4 \\
2.6 \\
2.8\end{array}$ & $\begin{array}{l}0.1 \\
0.2 \\
0.1 \\
0.1 \\
0.2\end{array}$ & $\begin{array}{l}11 \\
7.9 \\
7.9 \\
8.3 \\
7.9\end{array}$ & $\begin{array}{l}-- \\
-\overline{272} \\
-- \\
--\end{array}$ & $\begin{array}{l}290 \\
280 \\
280 \\
240 \\
300\end{array}$ & $\begin{array}{l}350 \\
270 \\
270 \\
270 \\
270\end{array}$ & $\begin{array}{l}<1 \\
<1 \\
<1 \\
1 \\
<1\end{array}$ & $\begin{array}{r}<10 \\
<3 \\
<3 \\
<10 \\
<3\end{array}$ \\
\hline-- & -- & -- & -- & -- & -- & -- & -- & -- & -- & -- & 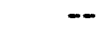 \\
\hline
\end{tabular}


Table 8.--Discharge and chemical quality

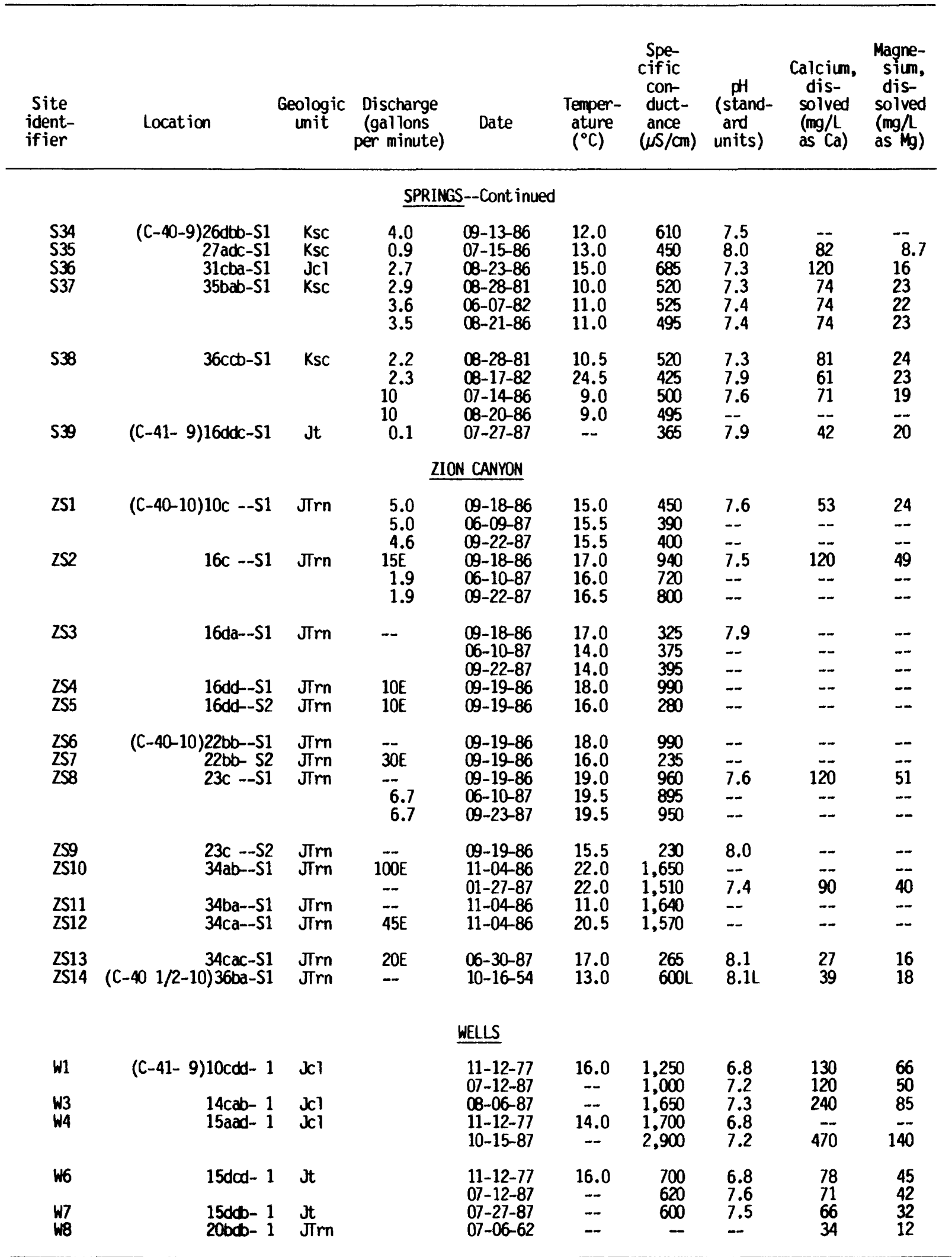


of water from selected springs and wells-Continued

\begin{tabular}{|c|c|c|c|c|c|c|c|c|c|c|c|}
\hline $\begin{array}{l}\text { Sodium, } \\
\text { dis- } \\
\text { solved } \\
\text { (mg/L } \\
\text { as } \mathrm{Na})\end{array}$ & $\begin{array}{l}\text { Potas- } \\
\text { sium, } \\
\text { dis- } \\
\text { solved } \\
\text { (mg/ } \\
\text { as K) }\end{array}$ & $\begin{array}{c}\text { Alka- } \\
\text { linity, } \\
\text { total } \\
\text { field } \\
(\mathrm{g} / \mathrm{L} \text { as } \\
\left.\mathrm{Cacm}_{3}\right)\end{array}$ & $\begin{array}{c}\text { Sulfate, } \\
\text { dis- } \\
\text { solved } \\
(\mathrm{mg} / \mathrm{L} \\
\left.\text { as } \mathrm{SO}_{4}\right)\end{array}$ & $\begin{array}{l}\text { Chlo- } \\
\text { ride, } \\
\text { dis- } \\
\text { solved } \\
\text { (mg/L } \\
\text { as Cl) }\end{array}$ & $\begin{array}{l}\text { Fluo- } \\
\text { ride, } \\
\text { dis- } \\
\text { solved } \\
(\mathrm{mg} / \\
\text { as } F)\end{array}$ & $\begin{array}{c}\text { Silica, } \\
\text { dis- } \\
\text { solved } \\
\text { (mg/L } \\
\text { as } \\
\mathrm{SiO}_{2} \text { ) }\end{array}$ & $\begin{array}{l}\text { Solids, } \\
\text { residue } \\
\text { at } 180 \\
\text { 'C, } \\
\text { dis- } \\
\text { solved } \\
\text { (mg/L) }\end{array}$ & $\begin{array}{l}\text { Solids, } \\
\text { sum of } \\
\text { consti- } \\
\text { tuents, } \\
\text { dis- } \\
\text { solved } \\
(\mathrm{mg} / \mathrm{L})\end{array}$ & $\begin{array}{l}\text { Hard- } \\
\text { ness } \\
(\mathrm{mg} / \mathrm{L} \\
\text { as } \\
\left.\mathrm{CaCO}_{3}\right)\end{array}$ & $\begin{array}{l}\text { Manga- } \\
\text { nese, } \\
\text { dis- } \\
\text { solved } \\
(\mu \mathrm{g} / \mathrm{L} \\
\text { as } \mathrm{Mn})\end{array}$ & $\begin{array}{l}\text { Iron, } \\
\text { dis- } \\
\text { solved } \\
(\mu \mathrm{g} / \mathrm{L} \\
\text { as } \mathrm{Fe})\end{array}$ \\
\hline \multicolumn{12}{|c|}{ SPRINGS--Cont inued } \\
\hline $\begin{array}{l}-- \\
2.1 \\
3.5 \\
3.2 \\
2.9 \\
3.1\end{array}$ & $\begin{array}{l}\overline{0.3} \\
1.6 \\
1.7 \\
1.3 \\
1.6\end{array}$ & $\begin{array}{l}254 \\
353 \\
190 \mathrm{~L} \\
260 \\
259\end{array}$ & $\begin{array}{l}-\overline{5.0} \\
20 \\
37 \\
31 \\
28\end{array}$ & $\begin{array}{l}1.3 \\
2.9 \\
2.7 \\
2.9 \\
2.4\end{array}$ & $\begin{array}{l}\ddot{0.1} \\
0.3 \\
0.1 \\
0.2 \\
0.2\end{array}$ & $\begin{array}{c}\overline{10} \\
15 \\
8.5 \\
8.0 \\
8.2\end{array}$ & $\begin{array}{l}\overline{242} \\
217 \\
-- \\
\overline{177}\end{array}$ & $\begin{array}{l}-\overline{260} \\
390 \\
260 \\
300 \\
300\end{array}$ & $\begin{array}{l}240 \\
370 \\
280 \\
280 \\
280\end{array}$ & $\begin{array}{l}\ddot{29} \\
33 \\
<1 \\
<1 \\
<1\end{array}$ & $\begin{array}{r}7 \overline{14} \\
4 \\
<10 \\
<3 \\
<3\end{array}$ \\
\hline $\begin{array}{l}2.1 \\
2.1 \\
1.8\end{array}$ & $\begin{array}{l}0.9 \\
1.2 \\
0.8\end{array}$ & $\begin{array}{l}150 \mathrm{~L} \\
260 \\
260\end{array}$ & $\begin{array}{l}24 \\
21 \\
16\end{array}$ & $\begin{array}{l}5.4 \\
2.0 \\
1.5\end{array}$ & $\begin{array}{l}0.1 \\
0.2 \\
0.1\end{array}$ & $\begin{array}{l}11 \\
11 \\
7.9\end{array}$ & $\begin{array}{l}\overline{-} \\
244\end{array}$ & $\begin{array}{l}240 \\
280 \\
270\end{array}$ & $\begin{array}{l}300 \\
250 \\
260\end{array}$ & $\begin{array}{r}1 \\
5 \\
<1\end{array}$ & $\begin{array}{r}<10 \\
9 \\
9\end{array}$ \\
\hline 2.6 & $\overline{2.5}$ & $\overline{176 L}$ & $\overline{10}$ & $\overline{2.9}$ & $\overline{0.2}$ & $\overline{9.3}$ & $\overline{196}$ & $\overline{200}$ & 190 & $\overline{1}$ & $\overline{3}$ \\
\hline \multicolumn{12}{|c|}{ ZION CANYON } \\
\hline 5.8 & 1.8 & 194 & 51 & 2.9 & 0.2 & 10 & 288 & 270 & 230 & $<1$ & 3 \\
\hline-- & -- & $\overline{-}$ & - & $=$ & -- & - & -- & - & -- & - & - \\
\hline 20 & $\overline{4.9}$ & 250 & 290 & $\overline{15}$ & $\overline{0.2}$ & 12 & $\overline{760}$ & 660 & 500 & 2 & 7 \\
\hline-- & - & -- & -- & - & -- & - & -- & - & -- & -- & -- \\
\hline-- & - & - & -- & -- & -- & - & -- & - & -- & -- & -- \\
\hline-- & -- & - & -- & - & -- & - & -- & -- & -- & -- & -- \\
\hline-- & -- & - & -- & -- & -- & - & -- & -- & -- & -- & -- \\
\hline- & - & - & -- & -- & - & - & -- & -- & -- & - & -- \\
\hline-- & -- & - & - & - & - & -- & -- & - & -- & -- & -- \\
\hline-- & -- & -- & -- & -- & -- & - & -- & -- & -- & -- & -- \\
\hline 23 & $\overline{--}$ & 230 & 310 & $\overline{15}$ & $\overline{0} 2$ & $\overline{13}$ & 670 & 680 & $\overline{510}$ & $=1$ & -5 \\
\hline- & 5.7 & - & -- & -- & -- & - & - & -- & -- & -- & -- \\
\hline-- & -- & - & -- & -- & -- & - & -- & -- & -- & -- & -- \\
\hline-- & -- & - & -- & -- & -- & - & -- & -- & -- & -- & -- \\
\hline 180 & $\overline{--}$ & $192 \mathrm{~L}$ & 280 & 260 & 0.3 & 14 & 1,000 & $\overline{990}$ & 390 & $\overline{<1}$ & 7 \\
\hline-- & 8.6 & - & - & - & -- & - & - & -- & - & -- & -- \\
\hline-- & -- & -- & -- & -- & -- & - & -- & -- & -- & -- & -- \\
\hline $\begin{array}{c}\overline{2.2} \\
54\end{array}$ & $\begin{array}{l}-- \\
4.4\end{array}$ & $\begin{array}{l}\overline{133 L} \\
128 L\end{array}$ & $\begin{array}{l}-\overline{4.7} \\
62\end{array}$ & 70 & $\begin{array}{l}--. \\
0.1 \\
0.1\end{array}$ & 10 & $\begin{array}{l}\overline{141} \\
340\end{array}$ & $\begin{array}{l}-- \\
140 \\
--\end{array}$ & $\begin{array}{l}-\overline{130} \\
170\end{array}$ & $\begin{array}{r}-- \\
--\end{array}$ & $\begin{array}{r}-- \\
--\end{array}$ \\
\hline \multicolumn{12}{|c|}{ WELLS } \\
\hline $\begin{array}{l}82 \\
40 \\
18\end{array}$ & $\begin{array}{l}1.8 \\
2.6 \\
8.9\end{array}$ & $\begin{array}{l}390 \mathrm{~L} \\
333 \mathrm{~L} \\
215 \mathrm{~L}\end{array}$ & $\begin{array}{l}270 \\
150 \\
660\end{array}$ & $\begin{array}{l}52 \\
55 \\
21\end{array}$ & $\begin{array}{l}1.1 \\
0.7 \\
0.1\end{array}$ & $\begin{array}{l}29 \\
21 \\
22\end{array}$ & $\begin{array}{r}\overline{707} \\
1,340\end{array}$ & $\begin{array}{r}870 \\
650 \\
1,200\end{array}$ & $\begin{array}{l}600 \\
510 \\
960\end{array}$ & $\begin{array}{r}<10 \\
3 \\
4\end{array}$ & $\begin{array}{r}30 \\
23 \\
8\end{array}$ \\
\hline$\overline{36}$ & $5 . \overline{4}$ & $\overline{173 L}$ & 1,500 & $\overline{79}$ & $\overline{1.8}$ & 35 & 2,890 & $2, \overline{370}$ & - & 40 & 30 \\
\hline $\begin{array}{c}15 \\
16 \\
13 \\
8.4\end{array}$ & $\begin{array}{l}2.0 \\
2.6 \\
2.1 \\
4.5\end{array}$ & $\begin{array}{l}270 \\
267 \mathrm{~L} \\
212 \mathrm{~L} \\
108 \mathrm{~L}\end{array}$ & $\begin{array}{r}130 \\
88 \\
81 \\
39\end{array}$ & $\begin{array}{l}13 \\
11 \\
11 \\
8.5\end{array}$ & $\begin{array}{l}0.5 \\
0.5 \\
0.4 \\
0.2\end{array}$ & $\begin{array}{l}14 \\
13 \\
11 \\
12\end{array}$ & $\begin{array}{l}-- \\
416 \\
403 \\
182\end{array}$ & $\begin{array}{l}460 \\
410 \\
340 \\
180\end{array}$ & $\begin{array}{l}380 \\
350 \\
300 \\
130\end{array}$ & $\begin{array}{r}<10 \\
1 \\
3 \\
--\end{array}$ & $\begin{array}{r}40 \\
5 \\
4 \\
1,300\end{array}$ \\
\hline
\end{tabular}


Pressure tests with inflatable straddle packers were used by Bingham Engineering (1980) to determine hydraulic-conductivity values (referred to as apparent permeability) during the dam-site investigation on the North Fork Virgin River. Packer tests were conducted in seven of the drillholes near IC2 and five of the drillholes downstream fram LC2 (Bingham Engineering, 1980, tables $C-1, F-1$ ).

About 100 pressure tests were made on the Navajo and Temple Cap aquifers. Drilling indicated that the degree of fracturing and friability was extremely variable in these aquifers, and hydraulic-conductivity values varied acoordingly. The largest value for hydraulic conductivity, about $120 \mathrm{ft} /$ day, was measured in a zone of friable sandstone. Values for fractured sandstone ranged from about 19 to $48 \mathrm{ft} /$ day; whereas, hydraulic-onductivity values for unfractured sandstone were two to three orders of magnitude smaller. Hydraulic-onductivity values for the brick-red siltstone at the base of the Temple Cap aquifer indicated that it is relatively impermeable compared to the overlying sandstone.

Values of hydraulic conductivity fram 40 packer tests for the limestone aquifer in the Carmel Formation ranged from $0.02 \mathrm{ft} /$ day in well cemented, silty sandstone to $78 \mathrm{ft} /$ day in fractured limestone and mudstone. The average value for relatively unfractured limestone was about $2 \mathrm{ft} /$ day; the average for shale was about $4.7 \mathrm{ft} /$ day.

Water levels measured in well LC4 following development are indicative of hydraulic conductivity in the lower coal-zone aquifer of the Tropic shale. Upon completion of the well, the water level was about $200 \mathrm{ft}$ below land surface. Water was purged from the well during development, and the water level immediately following purging was at a depth of about $350 \mathrm{ft}$. Two hours later, the water level had only recovered about $40 \mathrm{ft}$, indicating a poorly transmissive aquifer.

\section{Recharge}

Recharge to aquifers is mainly by infiltration of precipitation and seepage losses from streams in areas where the altitude exceeds 6,000 ft and average annual precipitation is greater than 12 in. (Cordova, 1981, p. 27). Recharge typically occurs over most of the study area where unconsolidated deposits mantle bedrock and rocks are made permeable by joints, fractures, solution cavities, or lava tubes and vesicles that collect seepage from precipitation and streams. Some of this water eventually recharges the older rocks such as the Navajo Sandstone (Cordova, 1981, p. 27). For example, recharge to the Navajo Sandstone from the Carmel Formation is evident from water-quality samples in the study area as discussed in the section on water quality.

Recharge also takes place locally in relatively permeable formations such as the Navajo Sandstone. On the basis of water-quality data, springs such as ZSl (fig. 9) that issue from fractures in the Navajo Sandstone, are probably recharged locally by direct infiltration of precipitation into fractures in the Navajo outcrop.

An unknown volume of subsurface inflow from the upper Sevier River basin (north of the lease tract and Navajo Lake) seeps from Navajo Lake (fig. 1) 
into solution cavities in the Wasatch Formation. This recharge flows to points of discharge such as Cascade Spring (about $1 \mathrm{mi}$ south of the southeast orner of Navajo Lake) in the upper Virgin River basin (Wilson and Thomas, 1964 , p. C24).

In the upper Virgin River basin (fig. 1), Cordova (1981, p. 28) estimated that about 4 percent of the normal annual precipitation is recharged to the ground-water system. Using this estimate and an average annual precipitation value of 22.5 in., the estimated annual recharge to the ground-water system in the lease-tract area is about 1,425 acre-ft. This is about 3 percent of the average annual ground-water recharge to the upper Virgin River basin of 55,000 acre-ft (Cordova, 1981, p. 28).

Most of the western part of the lease tract is underlain by the Tropic Shale, which probably accepts very little recharge. However, the Wahweap Sandstone, Kaiparowits Formation, and Straight Cliffs Sandstone exposed in the eastern part of the lease tract may accept more than four percent of average annual precipitation as recharge, particularly where they are jointed or fractured.

\section{Movement}

A potentiometric map for the regional water table in the Navajo aquifer could not be prepared because few water-level measurements are available for the study area. Cordova (1981, p. 28) described the direction of ground-water movement in the upper Virgin River basin as generally southward from the recharge areas in the northern highlands to areas of natural discharge along the lower reaches of larger streams.

The springs discharging from the walls of Zion Canyon are believed to be the natural discharge areas for the Navajo aquifer along the lower reaches of the North Fork Virgin River. The similarities in spring elevations on the east and west sides of Zion Canyon (fig. 15) and the relatively small change in spring elevations southward (a gradient of $50 \mathrm{ft} / \mathrm{mi}$ from zS3 to ZSl4) indicate that these springs represent points of the regional water table in the Navajo aquifer.

There probably is substantial vertical movement of water, primarily through fractures, in unsaturated rock in the area in order for water to recharge the Navajo aquifer and overlying aquifers. Any downward movement of water from the limestone aquifer of the Carmel Formation into the Temple Cap Sandstone is probably the result of flow in fractures. An unsaturated zone about 130 to $150 \mathrm{ft}$ thick between the Temple Cap aquifer (drillhole LC2) and the limestone aquifer in the Carmel indicates that flow through pore spaces is not occurring between the two strata. Drilling during this study indicates that both the limestone and the underlying sandstone are fractured.

The loss of circulation at a depth of $353 \mathrm{ft}$ during drilling in the Navajo Sandstone at LC2 is believed to be the result of fracturing. Furthermore, logs of cores from the dam-site investigation near LC2 (Bingham Engineering, 1980) showed joints or fracture zones in the Temple Cap Sandstone as well as fractures and solution cavities in the limestone member of the Carmel Formation. Fractures in the limestone member were generally filled 


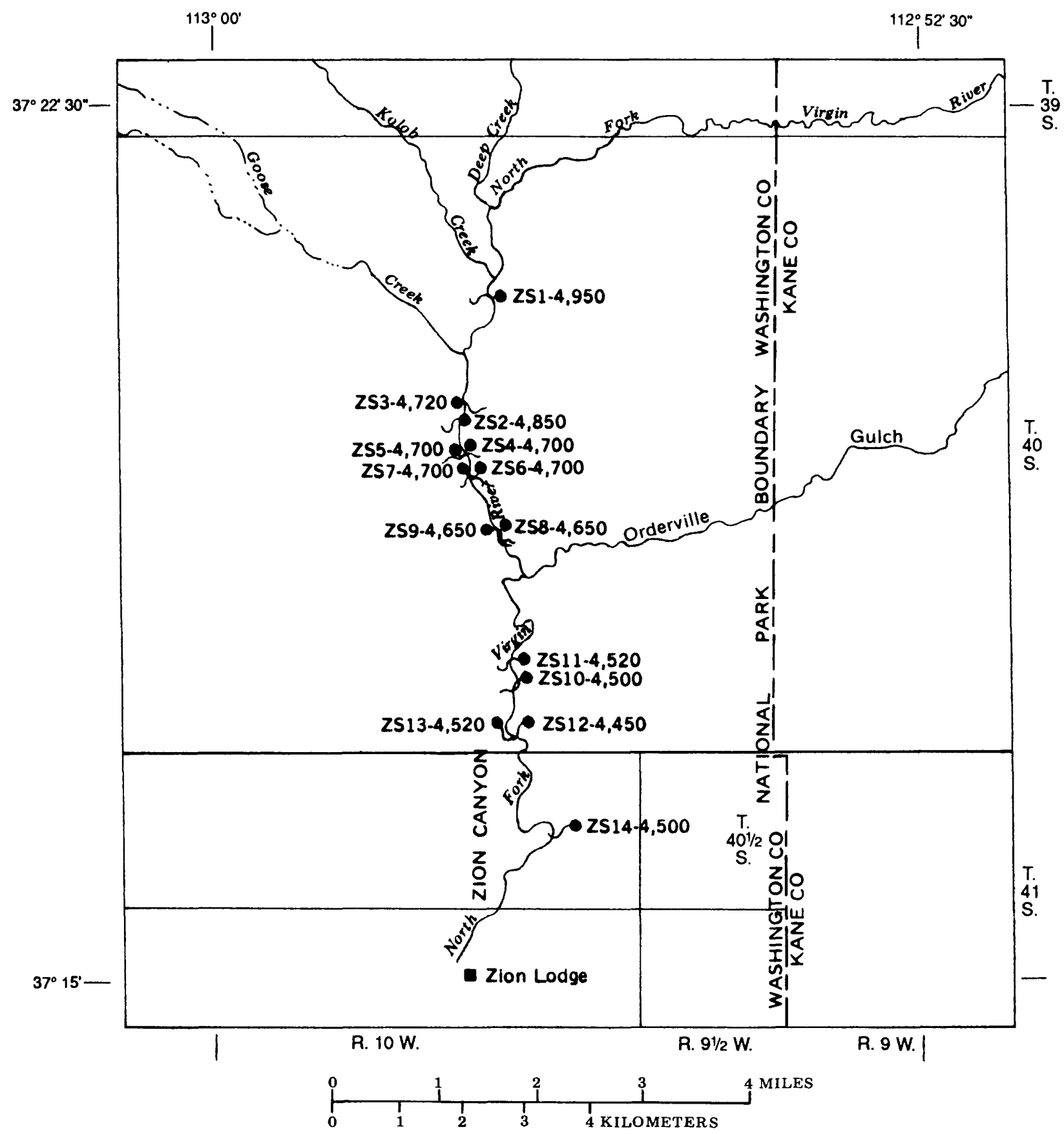

\section{EXPLANATION}

ZS14-4.500

SPRING--Number on left is identifier; number on right is elevation in feet above National Geodetic Vertical Datum of 1929.

Elevations are approximate

Figure 15.--Location and elevation of selected springs in Zion Canyon. 
with calcite or iron oxides. Zones of very friable sandstone (no core recovery) were also common in the Temple Cap Sandstone.

Fractures or friable zones in the Navajo Sandstone strongly influence the local direction of ground-water movement, particularly at or near the land surface (Cordova, 1981, p. 19); however, at depth, as in the Navajo aquifer, fractures and joints may be closed or partly closed and pore-space permeability may be more important. The prominent northwest-trending system of fractures and joints visible in the Navajo Sandstone at Zion National Park (fig. 5) may cause same ground water on a local scale to move perpendicular to the inferred southward direction of regional flow.

\section{Discharge}

In the study area, ground water is discharged from aquifers by diffuse seepage to streams, by springs, and by evapotranspiration. There are no pumped wells in the coal-lease tract; however, about $2 \mathrm{mi}$ south of the lease tract, there are several wells that provide water for domestic and agricultural use (fig. 14).

Seepage to streams, which includes spring discharge to streams, can be roughly estimated for 1986 by suming values of streamflow during base flow for each stream traversing the lease tract; North Fork Virgin River, the stream in Orderville Gulch, Clear Creek, and Meadow Creek. Values for base flow used in the seepage calculation were $11 \mathrm{ft}^{3} / \mathrm{s}$ (SW4) for North Fork Virgin River, $0.41 \mathrm{ft}^{3} / \mathrm{s}$ (SW21) for the stream in Orderville Gulch, $0.15 \mathrm{ft} / \mathrm{s}$ (average of SW25 and SW26) for Clear Creek, and $0.019 \mathrm{ft}^{3} / \mathrm{s}$ (average of SW28 and Sw29) for Meadow Creek (table 1). Estimated seepage was about 8,400 acre$\mathrm{ft} /$ year. This value is many times more than the recharge value of 1,425 acre$\mathrm{ft} /$ year for the least tract. Estimated seepage value includes ground-water inflow to streams derived from the area of each stream's drainage basin; whereas, the recharge for the lease-tract is determined for a much smaller area, namely the acreage of the lease tract only.

Discharges were measured or estimated for 37 springs in the study area shown on plate 1, and data are shown in table 8. In addition, discharge was measured or estimated at an additional 16 springs shown in figures 9 and 14, and data are given in table 8.

Discharge varies onsiderably from springs in different formations and within the same formation as shown in table 9. Although all springs within the study area were not measured, the number of springs in each formation and the total annual discharge from springs in a particular formation are indicative of the relative quantity of ground water moving through the formation.

Springs in the Straight Cliffs Sandstone had the largest total discharge and the largest range in discharge values (table 9). Only a few springs with relatively small discharges were found in the Tropic Shale and Carmel Formations and in Quaternary alluvium. The largest discharge from springs in the undivided Wahweap Sandstone-Kaiparowits Formation was 3 to 5 times larger than those in the Tropic Shale and Carmel Formations (table 9). 
Table 9.-Compilation of discharge data for springs in and adjacent to the L.C. Holding coal-lease tract

(Measurements of discharge from 1986 except where noted)

[gal/min, gallons per minute; acre-ft/yr, acre-feet per year; E, Estimated]

Geologic units: Qal, alluvium; Kwk, Wahweap Sandstone and Kaiparowits Formation; Ksc, Straight Cliffs Sandstone; Kts, shaly member of Tropic Shale; Ktc, coal-bearing member of Tropic Shale; Jcb, banded member of Carmel Formation; Jcl, limestone member of Carmel Formation; Jt, Temple Cap Sandstone; JTrn, Navajo Sandstone.

\begin{tabular}{lcccc}
\hline \multirow{2}{*}{$\begin{array}{c}\text { unit } \\
\text { unlogic }\end{array}$} & Number of springs & $\begin{array}{c}\text { Range } \\
\text { (gal/min) }\end{array}$ & $\begin{array}{c}\text { Total } \\
\text { (gal/min) }\end{array}$ & $\begin{array}{c}\text { Total annual } \\
\text { (acre-ft/yr) }\end{array}$ \\
\cline { 4 - 5 } Qal & 1 & - & 0.3 & - \\
Kwk & 5 & $.25-66$ & 75 & 121 \\
Ksc & 25 & $.2-280$ & 523 & 844 \\
Kts/Ktc & 5 & $.13-13$ & 32 & 52 \\
Jcb/Jcl & 2 & $2.7-20 \mathrm{E}$ & 23 & - \\
Jt & 1 & - & .1 & 1390 \\
JTrn & 9 & $5.0-100 \mathrm{E}$ & 1242 & 37 \\
\hline
\end{tabular}

11987 measurements included for springs ZS8 and ZS13.

In $\mathrm{Zion} \mathrm{Canyon,} \mathrm{springs} \mathrm{are} \mathrm{numerous} \mathrm{in} \mathrm{the} \mathrm{Navajo} \mathrm{Sandstone} \mathrm{and} \mathrm{issue}$ from bedding planes, diastems (depositional breaks), joints, and fractures in cliff faces. Hanging gardens of ferns are commonly associated with these springs. Discharges vary by several orders of magnitude, and the larger springs discharge into the North Fork Virgin River.

Discharge of ground water by evapotranspiration takes place in areas of phreatophyte growth--generally on the alluvial plains of perennial streams where the ground water is at or within a few feet of the land surface. In the study area, phreatophytes are found along the banks of streams and in the meadows adjacent to streams, particularly along the North Fork Virgin River.

Meadowgrass and trees such as cottonwood, willow, and Russian-olive are the principal phreatophytes. They grow largely in the upper reaches of the North Fork Virgin River and its tributaries. Although a quantitative value for evapotranspiration in the study area was not determined, Cordova (1981, $p$. 34 ) reported an estimated annual evapotranspiration of 3,200 acre-ft for the Virgin River including the North and East Forks. Infrared photography 
indicates that the major areas of phreatophytes are on the Virgin River, East Fork Virgin River, and North Fork Virgin River in the southern part of Zion Canyon (Cordova, 1981, pl. 1). Thus, the quantity of discharge by evapotranspiration in the lease-tract area is a very small part of the 3,200 acre-ft.

\section{Water Quality}

The quality of ground water in the study area is largely dependent upon the availability of soluble substances in the rocks through which the water moves and the length of time the water is in contact with these soluble substances. Chemical analyses of water from springs and wells in the study area are shown in tables 8 and 10 .

The general chemical quality of water from 53 springs and 6 domestic wells was analyzed during this study (table 8). In addition, waters from seven springs and four wells were analyzed for dissolved metals (table 10). Water issuing from a spring in the Navajo Sandstone in Zion Canyon (zS10, table 10) was analyzed for stable isotopes.

Springs and wells in the Wasatch Formation were not sampled during this study because of the minor occurrence of this formation in the study area. A brief description of water quality for the Wasatch Formation is warranted, however, because springs issuing from the formation provide base flow at the headwaters of the North Fork Virgin River and some of its tributaries.

Two samples collected from springs in the Wasatch Formation by Cordova (1981, p. 43) had an average dissolved-solids concentration of $300 \mathrm{mg} / \mathrm{L}$. The major constituents were calcium, magnesium, and bicarbonate (Cordova, 1981, p. 44). The small dissolved-solids concentration and predominance of calcium, magnesium, and bicarbonate in the springs in the Wasatch Formation are reflected in the water quality of the North Fork Virgin River, as discussed in the section on surface-water quality.

Springs issuing from the undivided Wahweap Sandstone-Kaiparowits Formation have specific-conductance values ranging from 450 (S9) to 1,200 (S4) $\mu \mathrm{S} / \mathrm{cm}$ (table 8). Those with smaller conductance values occur near the top of these strata (Wahweap Sandstone), and the spring with the largest conductance value is near the base (Kaiparowits Formation, S4, pl. 1). Major ions are calcium and bicarbonate; however, magnesium also is a major constituent in the sample from $\mathrm{Sl}$, and sulfate is a major ion in the sample from 54.

Springs are abundant in the Straight Cliffs Sandstone; however, no wells in the area are completed in this formation. Specific-conductance values for springs in this formation range from 335 to $1,700 \mu \mathrm{S} / \mathrm{cm}$ (table 8 ). In those springs with conductivity values less than about $750 \mu \mathrm{S} / \mathrm{cm}$, calcium, magnesium, and bicarbonate predominate. Water-quality diagrams (pl. 1) indicate that, in general, sulfate is a major constituent when the conductivity exceeds $750 \mu \mathrm{s} / \mathrm{cm}$. The larger conductance values and sulfate concentration of water issuing from some springs may reflect water that has traveled through coal zones or shale beds in the Straight Cliffs. 
Table 10.-Chenical analyses for dissolved metals and stable isotopes

[A1] dissolved-metal concentrations

Location: See explanation for hydrologic data-site numbering system in text.

Isotopic analyses: $\delta \mathrm{D}$, ratio of ${ }^{2} \mathrm{H} /{ }^{1} \mathrm{H} ; \delta^{18} 0$, ratio of ${ }^{18} 0 /{ }^{16} 0 ; 0 / 00$, permil or parts per

\begin{tabular}{|c|c|c|c|c|c|c|c|c|}
\hline $\begin{array}{l}\text { Site } \\
\text { ident- } \\
\text { ifier }\end{array}$ & Location & Date & $\begin{array}{l}\text { Barium, } \\
\text { dis- } \\
\text { solved } \\
\text { (as Ba) }\end{array}$ & $\begin{array}{l}\text { Beryl- } \\
\text { lium, } \\
\text { dis- } \\
\text { solved } \\
\text { (as Be) }\end{array}$ & $\begin{array}{l}\text { Cadmium, } \\
\text { dis- } \\
\text { solved } \\
\text { (as Cd) }\end{array}$ & $\begin{array}{l}\text { Chro- } \\
\text { mium, } \\
\text { dis- } \\
\text { solved } \\
\text { (as Cr) }\end{array}$ & $\begin{array}{l}\text { Cobalt, } \\
\text { dis- } \\
\text { solved } \\
\text { (as Co) }\end{array}$ & $\begin{array}{l}\text { Copper, } \\
\text { dis- } \\
\text { solved } \\
\text { (as Cu) }\end{array}$ \\
\hline \multicolumn{9}{|c|}{ SPRINGS } \\
\hline $\begin{array}{l}\text { S14 } \\
\text { S39 }\end{array}$ & $\begin{array}{l}(C-39-9) 33 a d a-S 1 \\
(C-41-9) 16 d d c-S 1\end{array}$ & $\begin{array}{l}07-28-87 \\
07-27-87\end{array}$ & 360 & $<0 . \overline{5}$ & $-\overline{1}$ & $-\overline{-}$ & $\begin{array}{l}-\overline{8} \\
<3\end{array}$ & $<-\overline{10}$ \\
\hline \multicolumn{9}{|c|}{ ZION CANYON } \\
\hline $\begin{array}{l}\text { ZS1 } \\
\text { ZS2 } \\
\text { ZS8 } \\
\text { ZS10 } \\
\text { ZS13 }\end{array}$ & $\begin{array}{r}(C-40-10) 10 c--S 1 \\
16 c=-S 1 \\
23 c--S 1 \\
34 a b--S 1 \\
34 c a c-S 1\end{array}$ & $\begin{array}{l}09-18-86 \\
09-18-86 \\
09-19-86 \\
01-27-87 \\
06-30-87\end{array}$ & $\begin{array}{l}53 \\
25 \\
23 \\
31 \\
--\end{array}$ & $\begin{array}{r}2 \\
1 \\
0.8 \\
<0.5 \\
--\end{array}$ & $\begin{array}{r}<1 \\
<1 \\
<1 \\
1 \\
--\end{array}$ & $\begin{array}{l}-- \\
-- \\
=- \\
--\end{array}$ & $\begin{array}{l}<3 \\
<3 \\
30 \\
<3 \\
--\end{array}$ & $\begin{array}{l}<10 \\
<10 \\
<10 \\
<10 \\
--\end{array}$ \\
\hline \multicolumn{9}{|c|}{ WELLS } \\
\hline $\begin{array}{l}\text { W1 } \\
\text { W3 } \\
\text { W6 } \\
\text { W7 }\end{array}$ & $\begin{array}{r}\text { (c-41-9) } 10 c d d-1 \\
14 c a b-1 \\
15 d c d-1 \\
15 d d b-1\end{array}$ & $\begin{array}{l}07-12-87 \\
08-06-87 \\
07-12-87 \\
07-27-87\end{array}$ & $\begin{array}{l}44 \\
- \\
70 \\
--\end{array}$ & $\begin{array}{r}<0.5 \\
<0.5 \\
---\end{array}$ & $\begin{array}{l}<1 \\
-- \\
<1 \\
--\end{array}$ & $\begin{array}{l}<5 \\
--5 \\
--\end{array}$ & $\begin{array}{l}<3 \\
-- \\
<3 \\
--\end{array}$ & $\begin{array}{r}50 \\
-- \\
<10 \\
--\end{array}$ \\
\hline
\end{tabular}

Ground water in the Tropic Shale and Carmel Formation is in contact with easily dissolved minerals such as gypsum $\left(\mathrm{CaSO}_{4} \star_{22} \mathrm{2H}_{2} \mathrm{O}\right.$ ) and mirabilite ( $\mathrm{NaSO}_{4}$ * $2 \mathrm{H}_{2} \mathrm{O}$ ) (Price, 1987 , p. 34). As a result, water issuing from springs in these formations may have magnesium, sodium, and/or sulfate as major ions, in addition to calcium and bicarbonate (pl. 1).

Few springs issue from the Tropic Shale. Specific conductance values for water from the shale member of the Tropic Shale range from 490 to $1,550 \mu \mathrm{S} / \mathrm{cm}$, and a value of $1,230 \mu \mathrm{S} / \mathrm{cm}$ was measured for a spring (Sl3) in the coal-bearing member of the Tropic Shale (table 8). Major constituents are calcium, magnesium, and bicarbonate in waters with smaller conductance values (Sll, S20, and S27, pl. 1); whereas, sodium, sulfate, and bicarbonate predominate in water with larger conductance values (S22, pl. 1).

Specific-conductance values of 685 and $2,450 \mu \mathrm{S} / \mathrm{cm}$ were measured for springs issuing from the limestone (Jcl) (S36) and banded (Jcb) (S14) members, respectively, of the Carmel Formation (table 8 ). The major constituents in the water from spring S36 (Jcl) were calcium and bicarbonate (pl. 1). In the water from spring S14 (Jcb), calcium and sulfate were the major constituents (pl. 1). 
in water from selected springs and wells

are in micrograms per liter]

thousand; SMOW, Standard Mean Ocean Water.

\begin{tabular}{|c|c|c|c|c|c|c|c|c|c|}
\hline $\begin{array}{l}\text { Lead, } \\
\text { dis- } \\
\text { solved } \\
\text { (as } \mathrm{Pb} \text { ) }\end{array}$ & $\begin{array}{l}\text { Lithium, } \\
\text { dis- } \\
\text { solved } \\
\text { (as Li) }\end{array}$ & $\begin{array}{l}\text { Nickel, } \\
\text { dis- } \\
\text { solved } \\
\text { (as Ni) }\end{array}$ & $\begin{array}{l}\text { Molyb- } \\
\text { denum, } \\
\text { dis- } \\
\text { solved } \\
\text { (as Mo) }\end{array}$ & $\begin{array}{l}\text { Silver, } \\
\text { dis- } \\
\text { solved } \\
\text { (as Ag) }\end{array}$ & $\begin{array}{l}\text { Stron- } \\
\text { tium, } \\
\text { dis- } \\
\text { solved } \\
\text { (as Sr) }\end{array}$ & $\begin{array}{l}\text { Van- } \\
\text { adium, } \\
\text { dis- } \\
\text { solved } \\
\text { (as V) }\end{array}$ & $\begin{array}{l}\text { Zinc, } \\
\text { dis- } \\
\text { solved } \\
\text { (as } Z n \text { ) }\end{array}$ & $\begin{array}{l}\text { SD, } \\
\text { O/OO } \\
\text { SMOW }\end{array}$ & $\begin{array}{l}\delta^{18} 0, \\
\text { o/oo } \\
\text { SMOW }\end{array}$ \\
\hline \multicolumn{10}{|c|}{ SPRINGS } \\
\hline$<-\overline{10}$ & $-\overline{6}$ & $<-\overline{10}$ & $<\overline{10}$ & $-\overline{<1}$ & $\begin{array}{r}3,200 \\
86\end{array}$ & $\begin{array}{l}-\overline{6} \\
<6\end{array}$ & $-\overline{5}$ & -- & -- \\
\hline \multicolumn{10}{|c|}{ ZION CANYON } \\
\hline $\begin{array}{l}<10 \\
<10 \\
<10 \\
<10 \\
--\end{array}$ & $\begin{array}{l}12 \\
30 \\
49 \\
--\end{array}$ & $\begin{array}{l}-- \\
-- \\
-- \\
--\end{array}$ & $\begin{array}{l}<10 \\
<10 \\
<10 \\
<10 \\
--\end{array}$ & $\begin{array}{l}-- \\
-- \\
-- \\
-- \\
--\end{array}$ & $\begin{array}{r}680 \\
1,700 \\
1,800 \\
1,500 \\
98\end{array}$ & $\begin{array}{l}<6 \\
<6 \\
<6 \\
<6 \\
--\end{array}$ & $\begin{array}{r}8 \\
12 \\
12 \\
20 \\
--\end{array}$ & $\begin{array}{c}-- \\
-- \\
-104.5 \\
--\end{array}$ & $\begin{array}{c}-- \\
-- \\
-14.25 \\
--\end{array}$ \\
\hline \multicolumn{10}{|c|}{ WELLS } \\
\hline$<10$ & 30 & $<10$ & $<10$ & 2 & 7,500 & $<6$ & 93 & -- & -- \\
\hline$<10$ & 22 & $<10$ & $<10$ & $<1$ & 1,700 & $<6$ & 260 & -- & -- \\
\hline-- & -- & -- & -- & -- & 1,200 & -- & -- & -- & -- \\
\hline
\end{tabular}

Dissolved-solids concentrations for water from wells W1, W3, and W4, completed in the limestone member of the Carmel Formation, ranged from 650 to $2,370 \mathrm{mg} / \mathrm{L}$, and specific-conductance values ranged from 1,000 to $2,900 \mu \mathrm{S} / \mathrm{cm}$ (table 8). Major constituents varied with dissolved-solids concentration. Calcium and bicarbonate predominated in the water from Wl (fig. 14). Calcium and sulfate were major constituents in water from W3 (fig. 14), which is open to much of the thickness of the limestone member, and water from W4.

In contrast to water from aquifers in the Carmel Formation, water in the Temple Cap aquifer had specific-conductance values that ranged from 365 to 700 $\mathrm{mg} / \mathrm{L}$ and dissolved-solids concentrations that ranged from 200 to $460 \mathrm{mg} / \mathrm{L}$. Major constituents in the water included calcium, magnesium, and bicarbonate (fig. 14).

Although the Navajo Sandstone is in the subsurface in most of the study area, data on the quality of water in this formation is necessary in order to determine the sources of recharge to the water-table aquifer in the Navajo and the potential effects of mining on the quality of recharge to the Navajo. Ground-water samples from springs issuing from the Navajo Sandstone were collected at sites in Zion Canyon (fig. 9). Results of chemical analyses are given in tables 8 and 10 . 
Springs in the Navajo Sandstone with the smallest specific-conductance values are those discharging on the west side of $\mathrm{Zion}$ Canyon. As noted earlier, these springs represent discharge from the regional water-table aquifer. Specific conductance ranged from 230 to $325 \mu \mathrm{S} / \mathrm{cm}$ (table 8 ) for west-side springs in september 1986. Chemical analyses of a single west-side spring sample indicated calcium, magnesium, and bicarbonate were the predominant ions (ZS13, fig. 9).

In contrast, springs discharging from the Navajo aquifer on the east side of Zion Canyon, in most cases, had specific-conductance values several times larger than those on the west side as shown in figure 16. Specificconductance values for water samples taken on the east side of the canyon in September 1986 ranged from $450 \mu \mathrm{S} / \mathrm{om}$ at the north end of Zion Canyon (ZSI) to $1,650 \mu \mathrm{S} / \mathrm{cm}$ north of the Temple of Sinawava (ZS10, table 8). Deuterium and oxygen isotope values for spring ZS10 (table 10) plotted near the meteoric water line (Craig, 1961) and indicated very little evaporation of precipitation occurred before recharge.

Aside from ZSl, the major ions in spring discharge from the east side of Zion Canyon include calcium, magnesium, sulfate, and bicarbonate. In addition, sodium and chloride content in the springs increases to the south, particularly at zSlo (fig. 9). Calcium, magnesium, and bicarbonate are the predaminant ions in water from ZSl, which may indicate a different source or a different flow path for recharge to this spring.

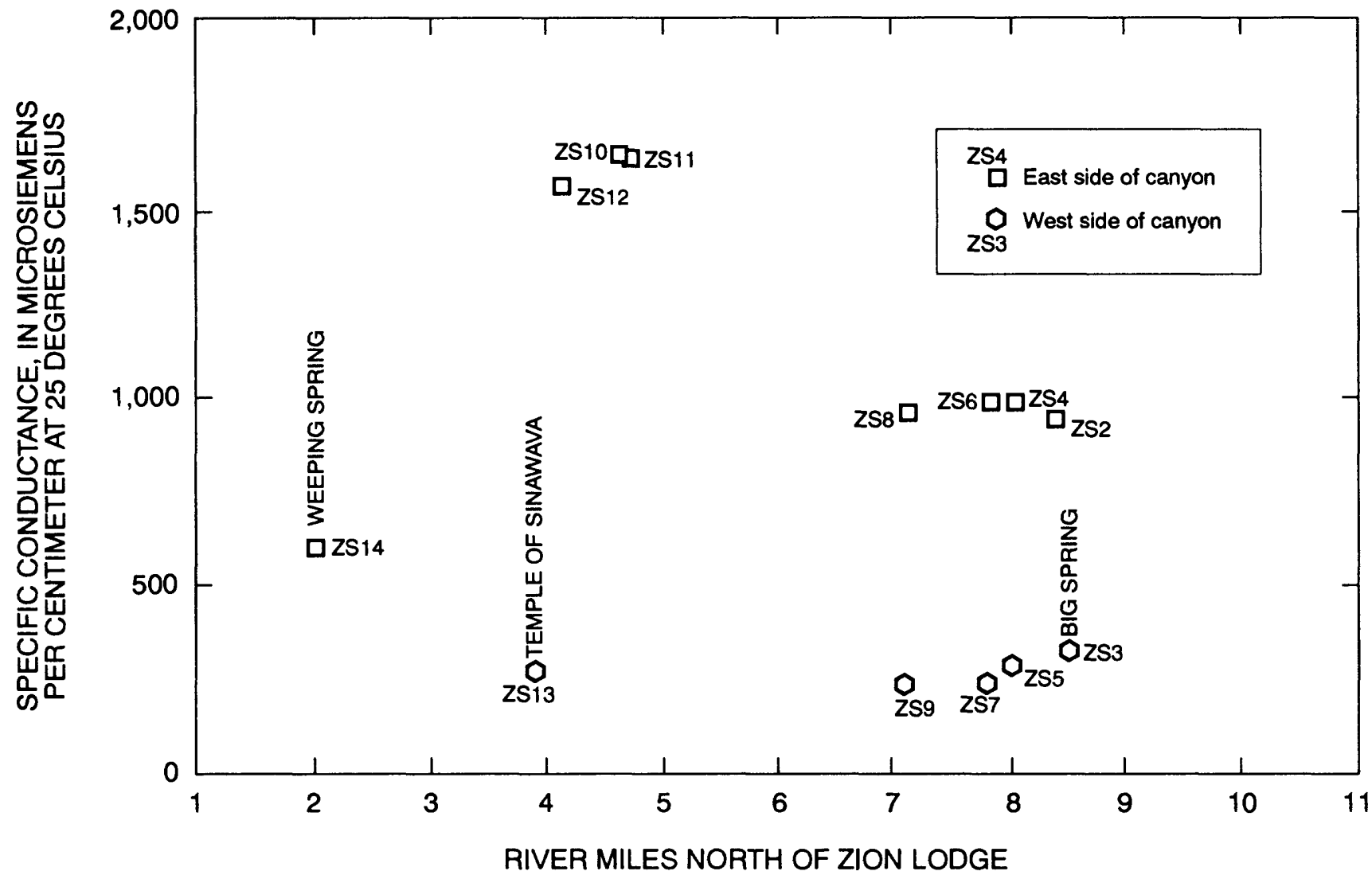

Figure 16.--Specific conductance of samples from springs discharging from the Navajo aquifer, Zion Canyon. 
The generally larger conductance waters issuing from east-side springs in the Navajo aquifer cannot be explained by slow movement through the formation. The Navajo Sandstone is a relatively pure quartz sandstone and probably adds little to the chemistry of the water (Cordova, 1978, p. 38). Instead, it appears that some of this water may be the result of recharge to the Navajo aquifer fram formations with water of greater specific conductance.

Strontium concentrations in ground water from the Carmel Formation, Temple Cap Sandstone, and Navajo Sandstone indicate that the Carmel Formation may be a major source of water that recharges the Temple Cap and Navajo Sandstones east of Zion Canyon. Large concentrations of strontium $(3,200$, 7,500 , to $7,700 \mu \mathrm{g} / \mathrm{L}$ ) were measured in water samples from the banded and limestone members of the Carmel Formation. Strontium is a major solute in seawater (Drever, 1982, p. 234) and concentrates in the calcite shells of marine organisms, which eventually settle to the ocean floor and form limestone. Some of the limestone beds in the limestone member of the Carmel Formation contain abundant shallow-marine fossils (Gregory, 1950, p. 93), which may be a source of strontium.

East of Zion Canyon, where the Carmel Formation blankets most of the area, relatively large concentrations of strontium $(1,200$ to $1,800 \mu \mathrm{g} / \mathrm{L})$ were measured in water from wells ( $W 6$ and $W 7$ ) in the Temple Cap aquifer and in springs (ZS2, ZS8, and ZS10) discharging from the Navajo aquifer into Zion Canyon. On the west side of Zion Canyon where the areal extent of the Carmel Formation is limited compared to that on the east side, a strontium concentration of $98 \mu \mathrm{g} / \mathrm{L}$ was measured in water from a spring (zS13) discharging from the Navajo aquifer. Spring S39, which discharges from the Temple Cap aquifer in an area devoid of Camel Formation overburden, has a strontium concentration of $86 \mu \mathrm{g} / \mathrm{L}$ (table 10 ).

The larger strontium concentrations in ground-water samples from wells in the Temple Cap aquifer, east of Zion Canyon, and springs discharging from the Navajo aquifer on the east wall of Zion Canyon, indicate that some ground water from the Carmel moves down to these aquifers. Conversely, the small strontium and dissolved-solids concentrations in springs on the west side of Zion Canyon, where the Navajo Sandstone is exposed at the surface, indicate recharge may, in part, be by direct infiltration into outcrops of the Navajo Sandstone.

Water moves to the regional aquifer by flow in fractures, solution cavities, lava tubes, and other natural conduits. Apparently, any recharge from the Carmel Formation to the Navajo aquifer on the west side of the canyon is minimal, as indicated by the lack of strontium and the small values of specific conductance. Alternatively, water recharging the Navajo aquifer in the west may not be in contact with the overlying Carmel Formation long enough to incorporate substantial dissolved solids.

The abundance of sulfate and occurrence of sodium and chloride in springs on the east side of the canyon (ZS2, ZS8, ZS10, and ZS14) also indicate recharge to the Navajo aquifer from overlying formations, particularly the Carmel Formation. Samples of water from wells completed in the limestone member of the Carmel Formation, east of $\mathrm{Zion}$ Canyon, had relatively large concentrations of sodium, chloride, and sulfate. Sodium concentrations in 1987 were $18 \mathrm{mg} / \mathrm{L}$ (W3), $36 \mathrm{mg} / \mathrm{L}$ (W4), and $40 \mathrm{mg} / \mathrm{L}$ (Wl), and chloride 
concentrations were $21 \mathrm{mg} / \mathrm{L}, 79 \mathrm{mg} / \mathrm{L}$, and $55 \mathrm{mg} / \mathrm{L}$, respectively (table 8 ). The sulfate concentration was greatest, $1,500 \mathrm{mg} / \mathrm{L}$, in well W4; however, water in this well probably was not representative of the aquifer because of reported contamination (Harold Drews, well owner, oral commun., 1987). The sulfate concentration was $150 \mathrm{mg} / \mathrm{L}$ in well Wl and $660 \mathrm{mg} / \mathrm{L}$ in W3.

Well W8, at the east entrance to Zion National Park, is almost $900 \mathrm{ft}$ deep and is completed in the Navajo aquifer (Gates, 1965, p. 156). The dissolved-solids concentration of water from w8 is comparable to that of springs discharging from the west side of Zion Canyon. The small dissolvedsolids concentration of water from well W8 may indicate that this well intercepts water along a different flow path than that of springs with large dissolved-solids concentrations on the east side of Zion Canyon.

\section{Ground-Water Inflow to Zion Narrows}

Because of the potential for mining in the L.C. Holding coal-lease tract to affect surface and ground water in adjacent areas, it is necessary to determine the source of base flow in the North Fork Virgin River as it passes through Zion National Park. The North Fork Virgin River is a major source of water to the plants and animals in the park. The river supports the Virgin River spinedace (Lepidomeda mollispinis mollispinis), a fish being considered for the endangered species list, and springs discharging from the canyon walls support vegetation that is the habitat for the endangered Zion snail (Physa zionis), found only in the park (Larry Hays, Zion National Park, oral commun., 1987). The river also adds to the beauty of Zion National Park, which attracts about 2 million tourists annually.

Data on streamflow and spring discharge as well as chemical quality were collected in Zion Canyon, particularly in Zion Narrows, the part of $\mathrm{Zion}$ Canyon north of the Termple of Sinawava (fig. 9). Data were collected in Zion Canyon to (1) determine the quantity of ground-water inflow to the North Fork Virgin River in Zion Narrows; and (2) determine, if possible, the relative quantity of inflow from sources on the east and west sides of the canyon and its relation to the lease tract. The locations of springs and streamflow sites in Zion Canyon are shown in figure 9.

\section{Quantity of Inflow}

Streamflow measurements on the North Fork Virgin River were made in September 1986 and June and September 1987. Data are shown in table 1. Determinations of ground-water inflow were made from the September measurements when streamflow was largely base flow consisting of spring discharge and ground-water seepage to the river. Generally, discharge measurements during a seepage study are made in one day, as near as possible to the same time, to minimize daily and diurnal variations in discharge. However, the access to Zion Narrows is on foot from either end of Zion Canyon, and 2 to 4 days were required for seepage and spring measurements. Daily and diurnal variations in streamflow on the North Fork Virgin River were assumed to be negligible during the September measurements.

Streamflow of $6.6 \mathrm{ft}^{3} / \mathrm{s}$ was measured on September 16, 1986, at site $\mathrm{ZSWI}$, the contact between the Temple Cap Sandstone and the Carmel Formation. On September 17, 1986, at site ZSW2, the flow was $6.7 \mathrm{ft}^{3} / \mathrm{s}$. Assuming no 
evapotranspiration or tributary inflow and that storage in alluvial material remained constant, ground-water inflow to the North Fork Virgin River along this reach was minimal.

The total discharge from Zion Narrows measured at ZSW9 was $36 \mathrm{ft}^{3} / \mathrm{s}$ on September 19, 1986 (table 1). Tributary inflow measured during september 1719, 1986, was $10.8 \mathrm{ft}^{3} / \mathrm{s}-9.1 \mathrm{ft}^{3} / \mathrm{s}$ Erom Deep Creek (ZSW3) on September 17, $0.21 \mathrm{ft}^{3} / \mathrm{s}$ from Goose Creek (ZSW5) on September 18, and $1.5 \mathrm{ft}^{3} / \mathrm{s}$ from Orderville Gulch (ZSW8) on September 19. Kolob Creek, which is regulated, was not flowing at this time.

The data from the September 1986 seepage study indicate that about 18.6 $\mathrm{ft}^{3} / \mathrm{s}$ of ground water from springs and seepage entered the North Fork Virgin River between the oonfluence with Deep Creek (ZSW2) and the Temple of Sinawava (ZSW9) [ $36 \mathrm{ft}^{3} / \mathrm{s}$ at ZSW9 minus inflow fram tributaries $\left(10.8 \mathrm{ft}^{3} / \mathrm{s}\right.$ ) and flow in the North Fork Virgin River above Deep Creek $\left.\left(6.6 \mathrm{ft}^{3} / \mathrm{s}\right)\right]$. Evapotranspiration was considered negligible because of the lack of vegetation in Zion Narrows between ZSW2 and ZSW9, and storage in alluvium was assumed to remain constant.

The September 1986 data indicate that about 13,000 acre-ft/yr of the flow at ZSW9 is ground-water inflow from the Navajo aquifer. This value for inflow may vary seasonally (throughout the year) and annually (from year to year) based on the time and quantity of recharge. For 1986, 13,000 acre-ft/yr probably represents a minimum value for the year because base flow is considered to be lowest in the fall. The oontributions from spring discharge and seepage probably increase as recharge from snowmelt (spring) and thunderstorms (summer) reaches the Navajo aquifer.

Although 13,000 acre-ft/yr may represent a seasonal minimum value, it probably represents greater-than-normal inflow compared to previous years when precipitation was less. A graph of the cumulative departure from normal precipitation at Zion and Alton, 1967-87 (fig. 17), indicates that after a dry period in 1976-77, a wet period resulted in increasingly positive values of cumulative departure. Presumably, as precipitation increased, recharge to and discharge from the Navajo aquifer also increased.

\section{Source of Inflow}

The quantity of ground-water seepage or inflow that enters the North Fork Virgin River from the east and west sides of zion Canyon can be estimated using discharge and specific-onductance values. The ground-water inflow for the reach between ZSW6 and ZSW9 was estimated because more data were available for this reach. The relationship between streamflow at sites ZSW6 and ZSW8, ground-water inflow from the east and west sides of $\mathrm{Zion}$ Canyon, and the 


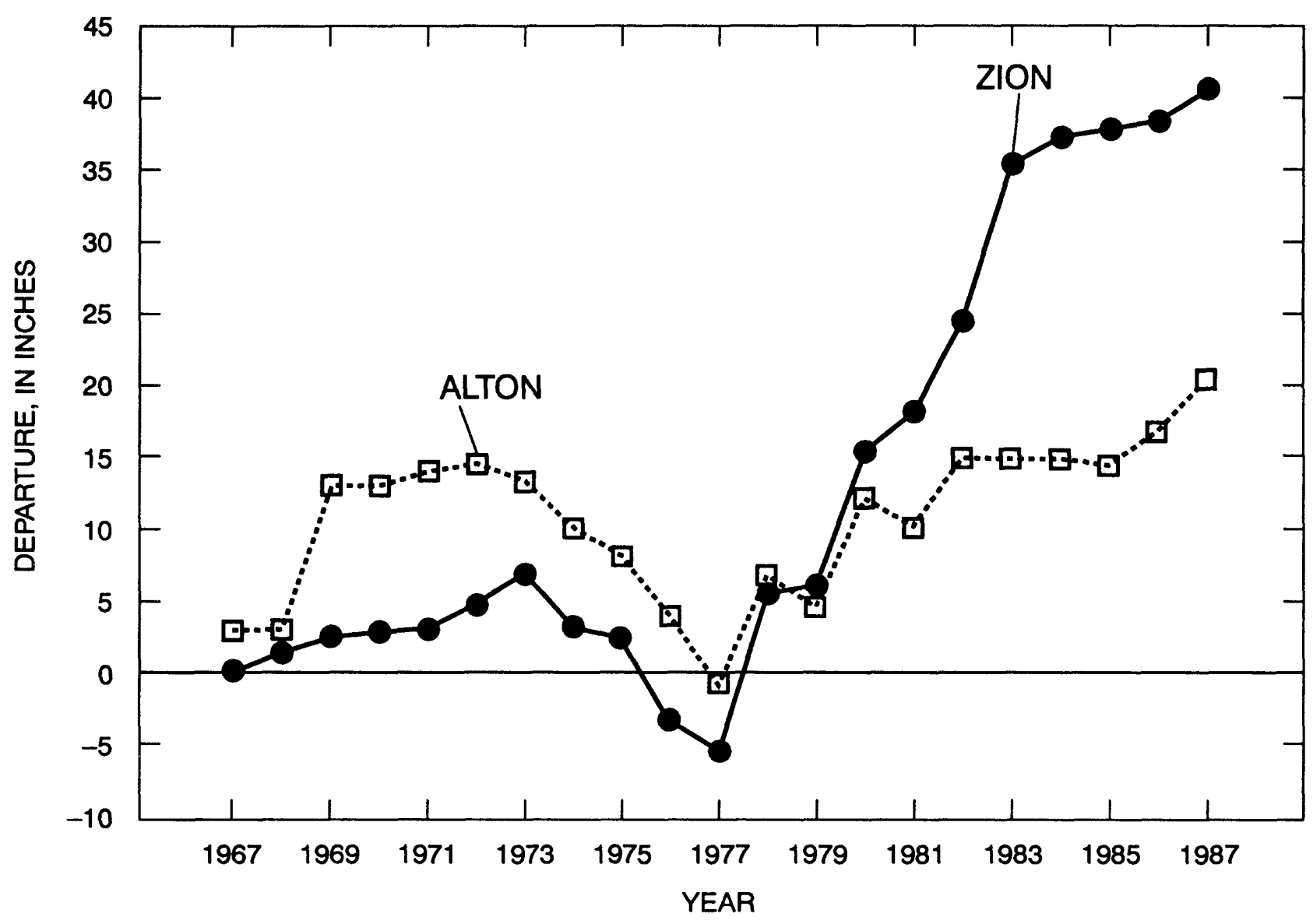

Figure 17.--Cumulative departure from normal precipitation at Zion and Alton, 1967-1987.

resulting mixture at the Temple of Sinawava (ZSW9) is given by:

$$
\mathrm{C6} Q 6+C 8 Q 8+C E Q E+C W Q W=C 9 Q 9
$$

where $\mathrm{C6}=$ specific conductance of streamflow at site zSW6, North Fork Virgin River near Big Spring;

Q6 = streamflow at site ZSW6;

C8 = specific conductance of streamflow at site ZSW8 on Orderville Gulch at mouth;

Q8 = streamflow at site ZSW8;

$\mathrm{CE}=$ average specific conductance of ground water from the east side of Zion Canyon;

$\mathrm{QE}=$ inflow of ground water from east side of Zion Canyon;

$\widetilde{C W}=$ average specific conductance of ground water from the west side of Zion Canyon;

$\mathrm{QW}=$ inflow of ground water from west side of Zion Canyon;

C9 = specific conductance of streamflow at site ZSW9, North Fork Virgin River at Temple of Sinawava; and

Q9 = streamflow at site $\mathrm{ZSW} 9$. 
Substituting

$$
Q E=Q 9-Q 6-Q 8-Q W
$$

into equation 1 and solving for $\mathrm{QW}$, the equation becomes

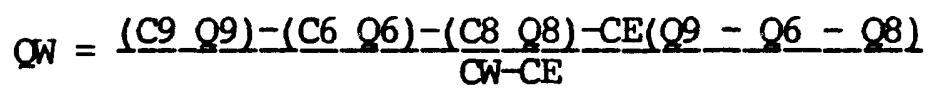

Values for $\mathrm{C} 6, \mathrm{Q6}, \mathrm{C} 8, \mathrm{Q8}, \mathrm{C} 9$, and $\mathrm{Q9}$ were determined from measurements made in September 1986 and given in table 1. Specific conductance values for ground-water inflow on the east (CE) and west (CW) sides of Zion Canyon were assumed to be equal to the average specific conductance measured for springs on the respective sides of the canyon. CE is estimated to be about $1,030 \mu \mathrm{S} / \mathrm{cm}$, and $\mathrm{CW}$ is estimated to be about $300 \mu \mathrm{S} / \mathrm{cm}$ using all specificconductance data from 1986 and 1987 for springs located along the river from ZSW6 to ZSW9. Solving for QW yields $2.65 \mathrm{ft}^{3} / \mathrm{s}$, and $Q E$ equals $4.85 \mathrm{ft}^{3} / \mathrm{s}$. Assuming that the measured specific conductance of the springs represents the general quality of diffuse seepage in this reach of the North Fork Virgin River, about 65 percent of the ground water entering the North Fork Virgin River from the Navajo aquifer in this reach is contributed by springs and seepage from the east side of Zion Canyon.

This type of analysis is usually done using conservative constituents such as chloride instead of specific conductance. However, chemical analyses were not available for the river and most springs on the west side of zion Canyon. Using conservative constituents instead of specific conductance could change the estimate of the relative contribution of ground water from the east and west sides of the canyon.

\section{SURFACE-WATER/GROUND-WATER INIERACTION}

Discharge measurements were made on the North Fork Virgin River and the stream in Orderville Gulch to identify gaining and losing channel reaches. Discharge measurements in November 1986 (table 1), between sites SWI and SW4 (pl. 2), indicate that about 62 percent of the flow $\left(6.8 \mathrm{ft}^{3} / \mathrm{s} ; \mathrm{Swl}\right.$, table 1) in the North Fork Virgin River at SW4 (11 $\mathrm{ft}^{3} / \mathrm{s}$; table 1 ) is derived upstream from the lease tract, above SWl. The stream in Bulloch canyon supplies about 32 percent $\left(3.5 \mathrm{ft}^{3} / \mathrm{s} ;\right.$ SWB, table 1$)$ and the remaining 6 percent $\left(0.7 \mathrm{ft}^{3} / \mathrm{s}\right)$ is the result of subsurface inflow and minor contributions from other tributaries.

The stream in Hay Canyon, which drains a substantially larger area than the stream in Bulloch Canyon, contributes only about $0.22 \mathrm{ft}^{3} / \mathrm{s}$ (SWI3, table 1) of the $0.7 \mathrm{ft}^{3} / \mathrm{s}$ that results from subsurface inflow and tributary contributions upstream from SW4. Bouldery, gravelly alluvium (Qal) in the stream channel, swampy areas between SWII and SWI4, and a decrease in the discharge between SW9 and SW11 measured in June 1986, indicate that streamflow in Hay Canyon is percolating into the coarse-grained alluvium, saturating the alluvium, and resulting in a loss of streamflow as the stream traverses the alluvium. 
A gain in flow was measured on November 5, 1986, (table 1) from site SW1 to SW2 along the North Fork Virgin River where it crosses the Straight Cliffs Sandstone. Apparent gains were measured in November 1986 on the North Fork Virgin River where the river crosses the Tropic Shale between SW2 ( $11 \mathrm{ft}^{3} / \mathrm{s}$, table 1) and SW3 (12 ft $3 / \mathrm{s}$, table 1). However, there are several diversions, return flows, and small tributaries between SW2 and SW3. Therefore, the apparent gains are believed to be a combination of measurement error and unmeasured inflow.

Similarly, in the stream in Orderville Gulch, the Tropic Shale contributes little, if any, to base flow. In November 1986, the streamflow at SW19 $\left(0.09 \mathrm{ft}^{3} / \mathrm{s}\right.$, table 1$)$ plus the tributary inflow at SW23 $\left(0.32 \mathrm{ft}^{3} / \mathrm{s}\right.$, table 1) largely issuing from springs in the Straight Cliffs Sandstone (pl. $1)$, was about equal to the streamflow at $5 w 20\left(0.39 \mathrm{ft}^{3} / \mathrm{s}\right.$, table 1) where the stream crossed the shaly member of the Tropic shale. As the stream crossed the coal-bearing member of the Tropic Shale from SW20 to SW21 $\left(0.41 \mathrm{ft}{ }^{3} / \mathrm{s}\right.$, table 1), the difference in measurements was within the range of measurement error of about 5 percent.

The number of springs in the Straight Cliffs Sandstone and Tropic Shale supports the conclusions drawn from the seepage studies. Many springs were found in the Straight Cliffs Sandstone, and a gain in flow was measured in the North Fork Virgin River where it crossed the formation. Conversely, very few springs were found in the Tropic Shale, and gains or losses in surface-water discharge across this unit were within the margin of measurement error.

Some seepage may be derived from the undivided Wahweap SandstoneKaiparowits Formation. However, it is considered minimal based on the general lack of springs in these formations and the small flows in tributaries, such as the stream in Orderville Gulch, which have headwaters in or flow across these strata.

Inflow from springs in the Wasatch Formation is a major contribution to surface water in the North Fork Virgin River above SWl. Wilson and Thomas (1964, p. C22) describe subsurface flow in solution channels in the Wasatch Formation limestone that recharges springs such as Cascade Spring. As stated earlier, Cascade Spring is the source of the North Fork Virgin River, and other tributaries to the North Fork also have their headwaters in the Wasatch Formation.

\section{POTENTIAL EFFECTS OF MINING}

In order to assess the potential effects of coal mining on the hydrologic system, it is necessary to have some understanding of the mine plan. The draft environmental impact statement prepared by the U.S. Bureau of Land Management (1986) presents a hypothetical mine plan. Mining in the lease tract would be by surface contour methods where the thickness of the overburden (rock overlying the coal) is less than $100 \mathrm{ft}$, and by underground methods where the thickness of the overburden exceeds $100 \mathrm{ft}$.

The potential area of surface mining, the western part of the coal-lease tract, extends from about Birch Creek Hollow (T. $40 \mathrm{~S} .$, R. $9 \mathrm{~W} .$, sec. 21) to the northern part of the lease tract. Total land-surface disturbance over the 
25-year life of the mine would be 1,500 acres, with a maximum disturbance at any one time of about 375 acres plus 40 acres for surface facilities (U.S. Bureau of Land Management, 1986). The North Fork road, which would be one of the major accesses for hauling coal, would require extensive reconstruction.

An estimated 8.6 acre-ft/yr of water would be required for mining-related activities and would be obtained from the mine, wells, and surface water (U.S. Bureau of Land Management, 1986). Any ground water encountered during mining would be diverted to settling ponds before discharge into natural drainages.

Underground mining methods would be used in the eastern part of the lease tract. A possible portal site would be in T. 41 S., R. 9 W., sec. 2. Water would be pumped from the mine to the portal and discharged into Little Meadow Creek. Unreclaimed surface disturbance would be about 65 acres causing a potential increase in sediment yield of 40 tons per year to area streams (U.S. Bureau of Land Management, 1986). Mining-related subsidence could affect 500 to 1,985 acres with the largest subsidence occurring where the overburden is thin, and the coal is thick (U.S. Bureau of Land Management, 1986).

\section{Surface Mining}

Hydrology

The area considered for surface mining would require about 8.6 acre-ft (5 $\mathrm{gal} / \mathrm{min}$ ) of water per year for mining-related activities. If the mined area itself could not provide the water needed, surface-water sources or wells would be needed outside the mine area. The North Fork Virgin River would be the most convenient source for surface water. Spring and well discharges indicate that the straight cliffs Sandstone, the Carmel Formation (particularly the limestone member), and the Temple Cap Sandstone oould be the units with the potential to supply adequate water to wells to meet mining requirements.

The mine pit could become a sink, intercepting ground water that currently flows through the lease tract. Aquifers in the overburden and the coal could be locally dewatered by mining. Dewatering could cause spring discharges to decrease or stop in the dewatered strata.

\section{Water Quality}

Water used in mining activities will eventually be discharged into local drainages or consumed. Though storage in settling ponds will allow much of the sediment to be removed, the chemical quality of the water discharging into local drainages may contain larger dissolved-solids oncentrations than that of the local stream or river, particularly in the case of the North Fork Virgin River.

Coal in the Kolob coal field has moderate to high sulfur content (Sargent and Hansen, 1976, p. 33). Samples from the Orderville area have a sulfur content ranging from 1.10 to 4.03 percent and averaging 2.21 percent (Doelling and Graham, 1972, p. 271). Removal of overburden will expose sulfur-bearing minerals associated with the coal such as pyrite $\left(\right.$ FeS $\left._{2}\right)$, to oxidation. Increased sulfate oncentrations and decreased $\mathrm{pH}$ values in mine discharge 
could result from the oxidation of sulfide minerals (Seiler and Baskin, 1988, p. 37).

If recharge infiltrates in the mined area, it could change the chemistry of ground water locally in the underlying aquifers. Recharge water percolating through mine tailings and disturbed strata at the mine pit could leach oxidized constituents from the mined area, causing a localized increase in dissolved-solids concentrations. Water quality in the aquifer in the Winsor Member of the Carmel Formation, directly beneath the mined area, could be affected. Increased dissolved-solids concentration in water of deeper aquifers could occur in the future, if recharge reaches greater depths.

The sediment load in local drainages could increase because of disturbance of the unstable Tropic Shale for road work and mining. The Tropic Shale is particularly susceptible to slope instability and landslides. It is easily weathered and unstable even in gentle slopes. Surface mining in the area could expose about 400 acres of disturbed, unvegetated Tropic Shale to runoff, erosion, and soil loss. Settling ponds for runoff from the mine area could prevent most sediment from reaching local drainages.

Slope instability could increase during and after road construction. Existing roads into the area are narrow, marked by switch-backs, and in some places, have steep grades. The North Fork road would need to be paved, widened, and switch-backs and steep grades removed for efficient coal transport (U.S. Bureau of Land Management, 1986). Road work and unstable road cuts could add to the suspended-sediment load in local drainages.

Aquatic life in local drainages including the North Fork Virgin River could be affected if average sediment loads increase. Aquatic life such as the Virgin River spinedace in Zion Canyon can tolerate the sporadic, large suspended-sediment concentrations caused by summer thunderstorms. The spinedace cannot tolerate warm water or large dissolved-solids concentrations (Hickman, 1987, p. 7). Larger sediment loads at current or lower streamflow levels could cause spawning areas to be covered with fine silt, suffocating the fish ova.

\section{Underground Mining}

The underground mine could (1) Act as a sink, dewatering aquifers in the cal and overburden, (2) cause increased sediment loads in local drainages near surface facilities, and (3) cause increased dissolved-solids concentrations and lower pH values in Little Meadow Creek where mine waters are discharged. In addition, the subsidence of strata above the mine could substantially affect the hydrology of the area.

\section{Land Subsidence Above Coal Mines}

The most important factors controlling the area, quantity, rate, and duration of subsidence at coal mines in the western United States are the geotechnical properties of the coal and the rock above and below the coal being mined, the topography and slope of the land surface, the mine geometry, and mining method (Dunrud, 1984, p. 171-179). 
As coal is mined, the overburden is no longer fully supported, and rocks are subjected to stresses produced by compression arches and flexure of subsiding strata (Dunrud, 1976, p. 1). These stresses cause fractures in the overburden which extend from the mine upward.

Subsidence fractures can extend from the roof of a mine to the land surface or into aquifers several hundred feet above the mine. Dunrud (1976, fig. 9) reported subsidence fractures and compression arches in a massive sandstone $900 \mathrm{ft}$ above mine workings at the Geneva mine in the Book Cliffs coal field, central Utah. Tension cracks in thin soils overlying bedrock fractured by subsidence have been documented above a mine in the Wasatch coal field of central Utah (Danielson and Sylla, 1983, p. 32; Thiros and Cordy, 1991).

Potential Effects of Subsidence in the Lease Tract

Land-surface subsidence above the underground coal mine has the greatest potential for affecting the hydrology of the lease tract and the adjacent areas. Slopes, drainage patterns, aquifer recharge, and the quality of surface and ground water could be permanently affected by subsidence.

As stated earlier, aquifers exist in the coal zone and in the strata above and below the coal. Subsidence fractures and faults extending into the overburden above the mine could breach and displace the rocks of the aquifers (Lee and Abel, 1983, p. 9) in the Straight Cliffs Sandstone and Wahweap Sandstone-Kaiparowits Formation, diverting water into the mine and locally dewatering the aquifers. The discharge from some springs in these formations could decrease or cease (Hotchkiss and others, 1980, p. 46).

Subsidence fractures that extend to the ground surface might intercept runoff or streamflow, diverting it into the mine (Hotchkiss and others, 1980, p. 46). Surface and ground water diverted into the mine would be discharged from a portal into Little Meadow Creek, thus diverting flow from the North Fork Virgin River and Orderville Gulch drainages to the drainage basin of Little Meadow Creek. The potential diversion of streamflow from the springs and tributaries that drain into the North Fork Virgin River, in particular, might decrease the quantity and quality of flow leaving the lease tract.

Subsidence and the surface instability it creates might increase the potential for landslides and slumps, particularly in the Tropic Shale above the mine. Increased ground instability might add more sediment to the streams draining the lease tract.

\section{FUTURE STUDIES NEEDED}

The potential effects of coal mining on the hydrology of the study area cannot be quantitatively predicted until the relation between the Navajo aquifer and the overlying aquifers is better understood. More data on the depth of the Navajo aquifer east of Zion National Park are needed in order to prepare a potentiometric map. The Navajo aquifer is at great depth and drilling of monitoring wells requires special methods and equipment; however, a few strategically placed monitoring wells in the Navajo aquifer could provide necessary data. 
Better definition of the sources of flow to the hanging gardens and seepage to the North Fork Virgin River in Zion National Park is needed in order to determine the potential effects of mining or any other type of upstream development on surface and ground water in the Park. More data on water quality and quantity would be useful to further define the sources of flow into Zion National Park.

\section{SUMMARY AND CONCLUSIONS}

The L.C. Holding coal-lease tract is part of the Kolob coal field in southwestern Utah. Coal resources are estimated to be 209 billion tons. The hydrology of the area was studied to determine the potential effects of coal mining on water resources in the lease tract as well as in adjacent Zion National Park to the west.

Formations exposed in the study area include, in ascending order, the Navajo Sandstone, Temple Cap Sandstone, Carmel Formation, the coal-bearing Tropic Shale, Straight Cliffs Sandstone, undivided Wahweap SandstoneKaiparowits Formation, and local occurrences of the Wasatch Formation, remnant gravels, basic extrusive rocks, and alluvial and landslide deposits. Although surface evidence of faulting is relatively rare in the lease tract, a major joint system exposed in the Navajo Sandstone in Zion National Park probably continues in the subsurface in the L.C. Holding lease tract. Joints in the Navajo and other formations are important as conduits for recharge.

The major perennial stream draining the area is the North Fork Virgin River, which flows through Zion Canyon and into the Virgin River. Runoff from deep snowpacks and thunderstorms contribute most of the flow to perennial streams. Base flow in the North Fork Virgin River and several of its tributaries is sustained by spring discharge and diffuse seepage. Peak instantaneous streamflows for the North Fork Virgin River are generally associated with summer thunderstorm runoff.

The chemical composition of the North Fork Virgin River is about the same from upstream to downstream sample sites despite the inflow of water with larger dissolved-solids concentrations from some tributaries. Dissolvedsolids concentrations range from 190 to $280 \mathrm{mg} / \mathrm{L}$, and major constituents are calcium, magnesium, and bicarbonate plus carbonate. Suspended-sediment concentrations fluctuate with discharge--the largest concentrations occur during runoff from intense thunderstorms in summer and fall.

The regional aquifer is in the Navajo Sandstone, and the water table is at a depth of $870 \mathrm{ft}$. Ground-water movement is generally southward. Ground water also occurs in the overlying formations. Data from wells, springs, and drilling indicate that saturated zones are present in the Temple Cap Sandstone; in the Winsor Member and the banded and limestone members of the Carmel Formation; in the basal coal-bearing member of the Tropic Shale; in the Straight Cliffs Sandstone; and in the undivided Wahweap Sandstone-Kaiparowits Formation. 
Very little hydrologic information is available for aquifers in the study area because of the lack of wells and aquifer tests. Data from laboratory and field tests on the Navajo aquifer provided specific-capacity values ranging from 0.03 to 23 (gal/min)/ft, an average hydraulic-conductivity value of 5 $\mathrm{ft} /$ day, and estimated transmissivity values ranging from 1,070 to 14,000 $\mathrm{ft}^{2} /$ day.

Ground water issuing from springs in formations overlying the Tropic Shale has specific conductance values ranging from 335 to $1,700 \mu \mathrm{S} / \mathrm{cm}$. In general, calcium, magnesium, and bicarbonate predominate. In contrast, ground water in the Tropic Shale and Carmel Formation is in contact with easily dissolved minerals, and magnesium, sodium, and/or sulfate are major constituents, in addition to calcium and bicarbonate.

The quality of surface and ground water in zion Canyon indicates that (1) Large strontium concentrations and larger specific-conductance values for water issuing from springs on the east side of the canyon represent recharge to the Navajo Sandstone from the Carmel Formation, and (2) about 65 percent of the ground water entering the North Fork Virgin River from springs and seepage in Zion Canyon comes from the Navajo aquifer, east of the canyon.

Potential effects from surface mining in the western part of the L.C. Holding coal-lease tract include: (1) The mine pit intercepting ground water that currently flows through the lease tract; (2) dewatering of aquifers in formations above the mine causing a decrease or cessation of flow to some springs; (3) water with increased sulfate concentrations and decreased pH values entering local drainages and infiltrating down into aquifers; and (4) an increase in suspended-sediment load in local drainages from road work and unstable road cuts. Aquatic life in local drainages might be affected if sediment loads, dissolved-solids concentrations, or water temperatures increased.

Land subsidence above the underground mine in the eastern part of the lease tract might result in subsidence fractures extending from the mine into overlying formations and ultimately to the land surface. These fractures might divert ground water into the mine, locally dewatering aquifers in the overlying formations. The diversion of surface and ground water into the mine and subsequent discharge into Little Meadow Creek would divert streamflow fram the North Fork and Orderville Gulch drainages. Subsidence could also increase the potential for slope instability, thus, increasing the sediment yield to local drainages. 


\section{REFERENCES CITED}

Bowers, W.E., and Strickland, P.M., 1978, Geophysical logs of 14 test holes drilled during 1977 in the Kolob coal field, Kane County, south-central Utah: U.S. Geological Survey Open-File Report 78-258, 44 p.

Bingham Engineering, 1980, Bulloch Dam-Preliminary design report for Utah Division of Water Resources: Salt Lake City, Utah, 46 p.

Cashion, W.B., 1961, Geology and fuels resources of the Orderville-Glendale area, Kane County, Utah: U.S. Geological Survey Coal Investigations Map C-49, 1 sheet.

- - 1967, Geologic map of the south flank of the Markagunt Plateau, northwest Kane County, Utah: U.S. Geological Survey Miscellaneous Geologic Map I494, scale 1:250,000.

Christensen, R.C., Johnson, E.B., and Plantz, G.G., 1986, Manual for estimating selected streamflow characteristics of natural-flow streams in the Colorado River Basin in Utah: U.S. Geological Survey Water-Resources Investigations Report 85-4297, 39 p.

Colton, R.B., 1976, Landslide hazards in Sargent, K.A., and Hansen, D.E., eds., General geology and mineral resources of the coal area of southcentral Utah: U.S. Geological Survey Open-File Report 76-811, 122 p.

Cordova, R.M., 1978, Ground-water conditions in the Navajo Sandstone in the central Virgin River basin, Utah: Utah Department of Natural Resources Technical Publication 61, 66 p.

- 1981, Ground-water conditions in the upper Virgin River and Kanab Creek basins area, Utah, with emphasis on the Navajo Sandstone: Utah Department of Natural Resources Technical Publication 70, 87 p.

Craig, H., 1961, Isotopic variations in meteoric waters: Science, v. 133, p. 1702-1703.

Danielson, T.W., and Sylla, D.A., 1983, Hydrology of coal-resource areas in the southern Wasatch Plateau, central Utah: Water-Resources Investigations Report 82-4009, 66 p.

Doelling, H.H., Davis, F.D., and Brandt, Cynthia, 1986, Kane County geology: Survey Notes, v. 20, no. 2, p. 3-9.

Doelling, H.H., and Graham, R.L., 1972, Southwestern Utah coal fields: Alton, Kaiparowits Plateau and Kolob-Harmony: Utah Geological and Mineral Survey Monograph Series, no. 1, 333 p.

Drever, J.I., 1982, The geochemistry of natural waters: Prentice-Hall, Inc., Englewood Cliffs, N.J., p. 335-345.

Dunrud, C.R., 1976, Some engineering geologic factors controlling coal mine subsidence in Utah and Colorado: U.S. Geological Survey Professional Paper 969, 39 p. 
- -1984, Coal mine subsidence-western United States in Holzer, T.L., ed., Man-induced land subsidence: Reviews in Engineering Geology, Volume VI, Geological Society of America, p. 151-194.

Farnsworth, R.K., Thompson, E.S., and Peck, E.L., 1982, Evaporation atlas for the contiguous 48 United States: National Oceanic and Atmospheric Administration Technical Report NWS 33, 27 p.

Freethey, G.W., 1988, Geohydrology of the Navajo Sandstone in western Kane, southwestern Garfield, and southeastern Iron Counties, Utah: U.S. Geological Survey Water-Resources Investigations Report 88-4040, 43 p.

Gates, J.S., 1965, Ground water in the Navajo Sandstone at the east entrance of Zion National Park, Utah in Goode, H.D., and Robison, R.A., eds., Geology and resources of south-central Utah: Utah Geological Society and Intermountain Association of Petroleum Geologists Guidebook No. 19, Salt Lake City, Utah, p. 151-160.

Gregory, H.E., 1950, Geology and geography of the Zion Park region, Utah and Arizona: U.S. Geological Survey Professional Paper 220, 200 p.

Hamilton, W.L., 1984, The sculpturing of Zion: Zion Natural History Association, Springdale, Utah, $131 \mathrm{p}$.

Hem, J.D., 1985, Study and interpretation of the chemical characteristics of natural water: U.S. Geological Survey Water-Supply Paper 2254, 263 p.

Hickman, T.J., 1987, Study of fishes in the Virgin River (Utah)--Annual report for 1986: Western Ecosystems, St. George, Utah, 25 p.

Hintze, L.F., 1988, Geologic history of Utah: Brigham Young University Geology Studies Special Publication 7, 202 p.

Hotchkiss, R.H., Israelsen, E.K., and Riley, J.P., 1980, Management of the hydrologic system in areas subject to coal mining activities: Utah Water Research Laboratory, Water Resources Planning Series UWRL/P.80/06, 84 p.

Jeppson, R.W., and others, 1968, Hydrologic atlas of Utah: Utah Water Resources Lab, Utah State University, Logan, Utah, 306 p.

Lee, F.T., and Abel, J.F., Jr., 1983, Subsidence from underground mining: Environmental analysis and planning considerations: U.S. Geological Survey Circular 876, 28 p.

Lines, G.C., and others, 1984, Hydrology of Area 56, Northern Great Plains and Rocky Mountain coal provinces, Utah: U.S. Geological Survey WaterResources Investigations Open-File Report 83-38, 69 p.

Lohman, S.w., 1979, Ground-water hydraulics: U.S. Geological Survey Professional Paper 708, $70 \mathrm{p}$. 
Maddox, J.D., Hart, G.E., and Hawkins, R.H., 1977, A water resource inventory of the North Fork of the Virgin River, Zion National Park: unpublished report, Department of Forestry and Outdoor Recreation, Utah State University, Logan, Utah, 53 p.

Miller, J.F., Frederick, R.H., and Tracey, R.J., 1973, Precipitation-frequency atlas of the western United States, Volume VI--Utah: National Oceanic and Atmospheric Administration Atlas 2, 67 p.

National Oceanic and Atmospheric Administration, 1975a, Climatologic data, April 1975, Utah: Asheville, N.C., v. 77, no. 4.

- -1975b, Climatologic data, August 1975, Utah: Asheville, N.C., v. 77, no. 8 .

- - 1981-86, Climatologic data, annual summary, Utah: Asheville, N.C., v. 8388 , no. 13 .

Pacific Southwest Inter-Agency Committee, 1968, Report of the Water Management Subcommittee on factors affecting sediment yield in the Pacific southwest area and selection and evaluation of measures for reduction of erosion and sediment yield: Pacific Southwest Inter-Agency Comittee, 25 p.

Peterson, Fred, and Pipiringos, G.N., 1979, Stratigraphic relations of the Navajo Sandstone to Middle Jurassic formations, southern Utah and northern Arizona: U.S. Geological Survey Professional Paper 1035-B, 43 p.

Plantz, G.G., 1985, Hydrologic reconnaissance of the Kolob, Alton, and Kaiparowits Plateau coal fields, south-central Utah: U.S. Geological Survey Hydrologic Investigation Atlas HA-684, 2 pl.

Price, Don, 1980, Map showing general chemical quality of surface water in the Alton-Kolob coal-fields area, Utah: U.S. Geological Survey Miscellaneous Investigations Series Map I-1235-A.

- -1983, Map showing selected surface-water data for the Alton-Kolob coalfields area, Utah: U.S. Geological Survey Miscellaneous Investigations Series Map I-1235-D.

- -1987, Hydrology of Area 57, Northern Great Plains and Rocky Mountain coal provinces, Utah and Arizona: U.S. Geological Survey Water-Resources Investigations Open-File Report 84-068, 63 p.

ReMillard, M.D., Andersen, G.C., Birdwell, G.A., and Hookano, E., Jr., 1984, Water resources data, Utah, water year 1984: U.S. Geological Survey Water-Data Report UI-84-1, 462 p.

Sandberg, G.W., and Sultz, L.G., 1985, Reconnaissance of the quality of surface water in the upper Virgin River basin, Utah, Arizona, and Nevada, 1981-82: Utah Department of Natural Resources Technical Publication 83, $69 \mathrm{p}$. 
Sargent, K.A., and Hansen, D.E., 1976, General geology and mineral resources of the coal area of south-central Utah: U.S. Geological Survey Open-File Report 76-811, 122 p.

Seiler, R.L., and Baskin, R.L., 1988, Hydrology of Alkali Creek and Castle Valley Ridge coal-lease tracts, central Utah, and potential effects of coal mining: U.S. Geological Survey Water-Resources Investigations Report 87-4186, 53 p.

Shown, L.M., 1970, Evaluation of a method for estimating sediment yield: U.S. Geological Survey Professional Paper 700-B, p. B245-B249.

Stiff, H.A., Jr., 1951, The interpretation of chemical water analysis by means of patterns: Journal of Petroleum Techniques, Technical Note 84, p. 15-17.

Stouffer, S.G., 1964, Landslides in the Coal Hill area, Kane County, Utah: umpublished Master's thesis, University of Utah, Salt Lake City, Utah, $102 \mathrm{p}$.

Thiros, S.A., and Cordy, G.E., 1991, Hydrology and effects of mining in the Quitchupah and Pines coal-lease tracts, central Utah: U.S. Geological Survey Water-Resources Investigations Report 90-4084, 63 p.

Thomas, B.E., and Lindskov, K.L., 1983, Methods for estimating peak discharge and flood boundaries of streams in Utah: U.S. Geological Survey WaterResources Investigations Report 83-4129, 77 p.

Turner, W.V., 1949, Watershed conditions in the upper Virgin River Basin, Utah: U.S. Bureau of Reclamation, Boulder City, Nevada, 42 p., 3 pl.

U.S. Bureau of Land Management, 1986, Draft L.C. Holding environmental impact statement: Cedar City, Utah.

U.S. Weather Bureau, 1963, Normal annual precipitation (1931-60) for the State of Utah: Washington, D.C., scale 1:500,000, 1 sheet.

Utah Division of Water Resources, 1979, White River Dam project, proposed action plan: Salt Lake City, Utah, 45 p.

Uygur, Kadir, 1980, Hydraulic and petrographic characteristics of the Navajo Sandstone in southern Utah: University of Utah, Department of Geology and Geophysics, unpublished Master's thesis, $134 \mathrm{p}$.

Wilson, M.T., and Thomas, H.E., 1964, Hydrology and hydrogeology of Navajo Lake, Kane County, Utah: U.S. Geological Survey Professional Paper $417-\mathrm{c}, 26 \mathrm{p}$. 
SUPPLEMENIAL INFORMATION 


\section{EXPLANATION FOR LITHOLOGIC LOGS}

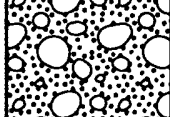

GRAVELS, COBBLES, BOULDERS - Dots indicate sand if present.

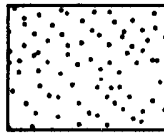

SANDSTONE

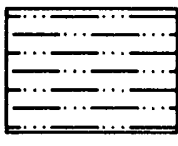

SILTSTONE

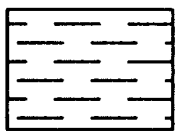

SHALE

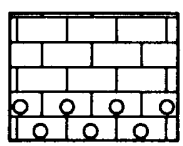

LIMESTONE - Circles indicate oolitic limestone

GYPSUM

COAL 


\section{Lithologic Log for Well LC1}

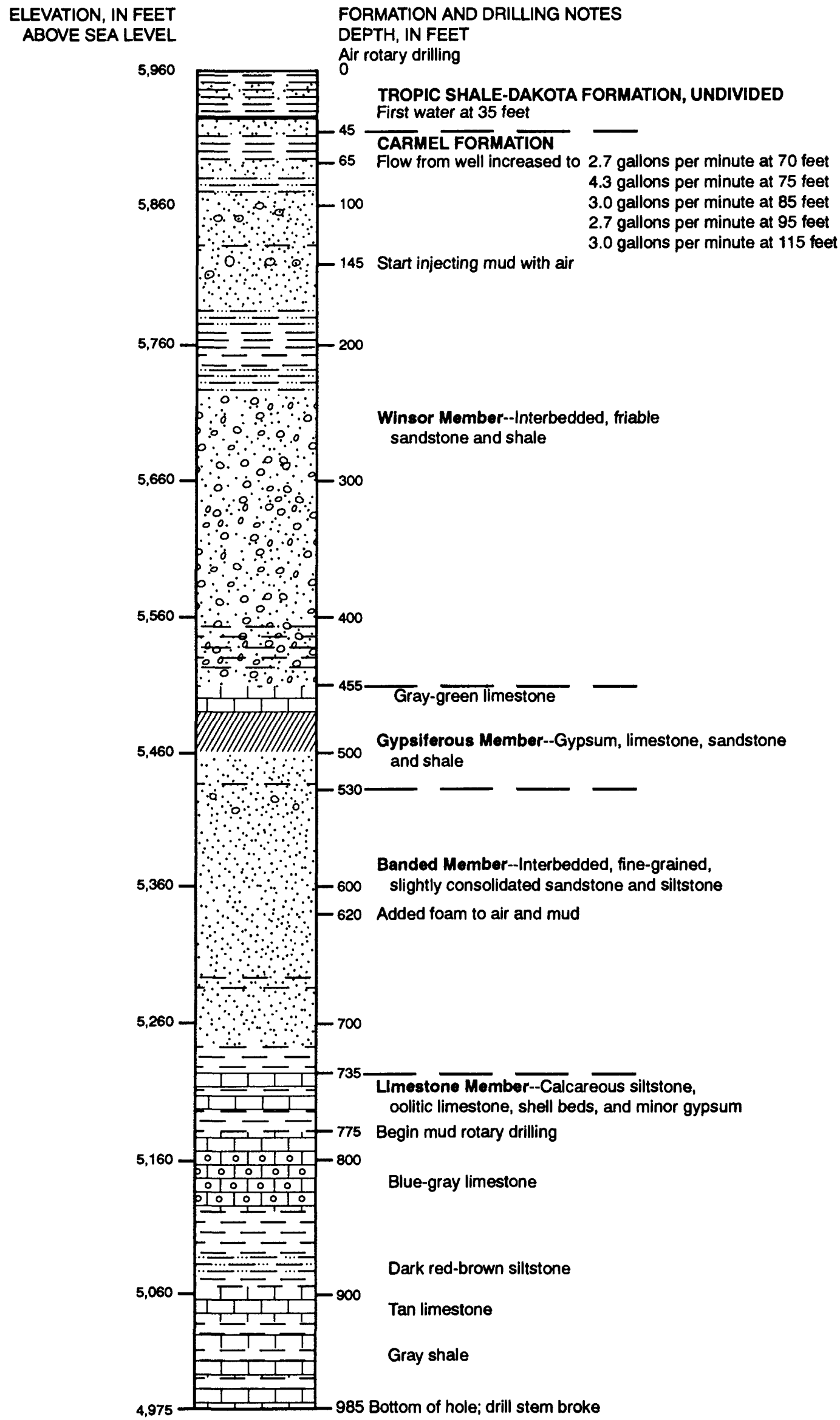


FORMATION AND DRILLING NOTES

DEPTH, IN FEET

Air rotary drilling

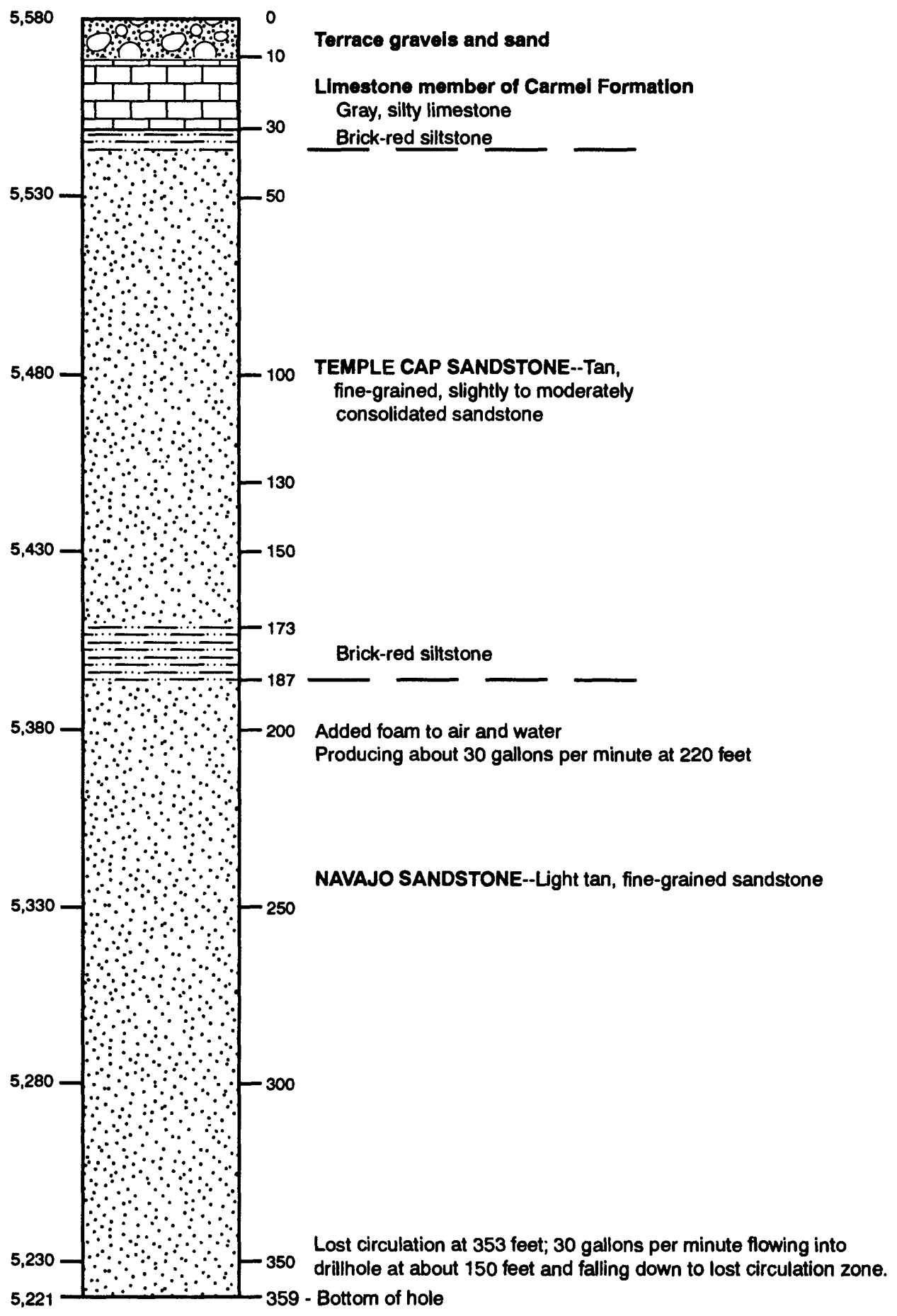




\section{Lithologic Log for Well LC3}

ELEVATION, IN FEET
ABOVE SEA LEVEL
FORMATION AND DRILLING NOTES
DEPTH, IN FEET
Tir rotary drilling




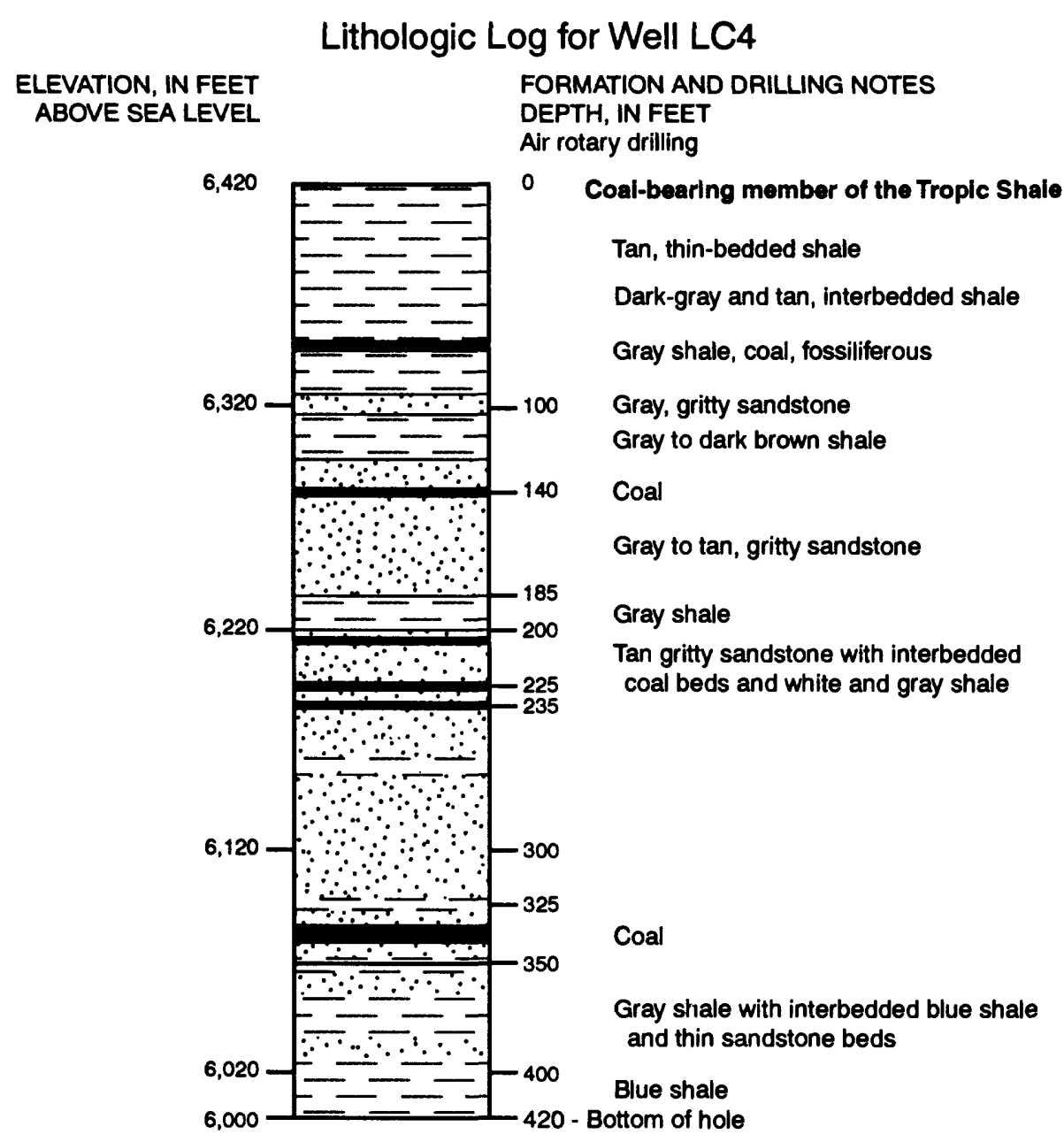

